Supporting Information

\title{
An approach for the generation of $\gamma$-propenylidene- $\gamma$-butenolides and application to the total synthesis of rubrolides
}

\author{
Debayan Roy, Prabhakararao Tharra, Beeraiah Baire* \\ Indian Institute of Technology Madras, Chennai-600036, Tamil Nadu, India. \\ E-mail: beeru@iitm.ac.in
}




\section{Table of Contents}

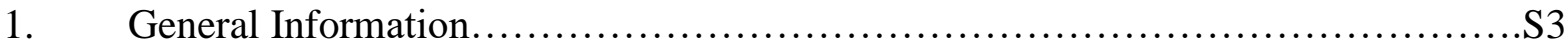

2. General procedure 1: General procedure for acid promoted allene formation.........S4

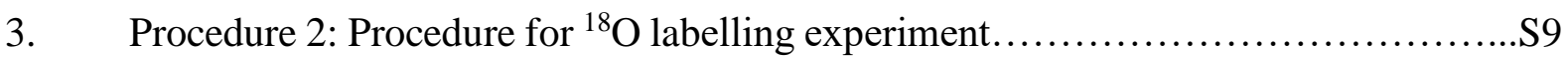

4. Procedure 3: Acid promoted allene formation in presence of oxygen...............S12

5. General procedure 4: Procedure for acid catalysed isomerisation of allenes.........S13

6. Procedure 5: Procedure for oxidative aromatization of triene 15a.................S15

7. General procedure 6: Procedure for synthesis of ( $\beta$-aryl- $\gamma$-alkylidene- $\gamma$-butenolides) derivatives from corresponding propargylic alcohols without isolation of intermediates.....S16

8. Procedure 7: Procedure for deprotection of methyl ether with boron tribromide......S19

9. General procedure 8: Procedure for Sonogashira reaction........................S19

10. Procedure 9: Procedure for preparation of propargyl alcohol 26...............S27

11. General procedure for preparation of vinyl iodides ...........................S28

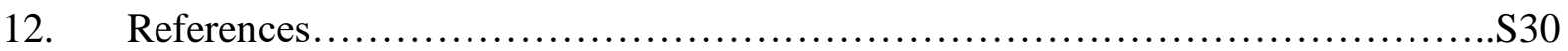

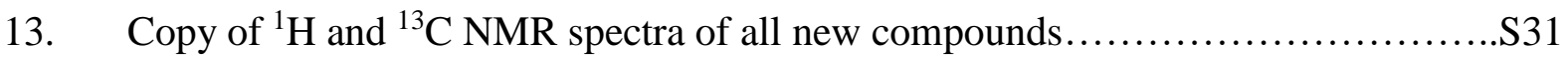




\section{General information}

All the solvents were distilled prior to use. Dry solvents were prepared according to the standard procedures. Commercially available chemicals were purchased from Sigma-Aldrich and Alfa Aesar and were used as received without further purification. Reactions requiring inert atmosphere were carried out under argon atmosphere. Infrared (IR) spectra were recorded on a JASCO 4100 FT-IR spectrometer. ${ }^{1} \mathrm{H}$ NMR spectra were measured on Bruker AVANCE $400 \mathrm{MHz}$ spectrometers. Chemical shifts were reported in ppm relative to solvent signals. ${ }^{13} \mathrm{C}$ NMR spectra were recorded on Bruker $100 \mathrm{MHz}$ spectrometers with complete proton decoupling. Chemical shifts were reported in ppm from the residual solvent as an internal standard $\left[\mathrm{CDCl}_{3} \delta=7.26 \mathrm{ppm}\right.$ for ${ }^{1} \mathrm{H}, \delta=77.16$ for ${ }^{13} \mathrm{C}$ or calibrated to tetramethylsilane $(\delta=$

0.00 ; DMSO- $d_{6} \delta=2.50 \mathrm{ppm}$ for ${ }^{1} \mathrm{H}, \delta=39.52$ for ${ }^{13} \mathrm{C}$ ). Peak multiplicities are designated by the following abbreviations: s, singlet; br s, broad singlet; $d$, doublet; $t$, triplet; q, quartet; m, multiplet; $J$, coupling constant in Hz. The coupling constant $J(\mathrm{~Hz})$ has been rounded to one decimal place for all compounds. Where a coupling pattern can be assigned as a combination of multiplicities, the above abbreviations have been combined to describe the observed patterns (i.e., dt - doublet of triplets). The high-resolution mass spectra (HRMS) were performed on Micromass QTOF micro mass spectrometer equipped with a Harvard apparatus syringe pump. For thin layer chromatography (TLC) analysis throughout this work, E-merck precoated TLC plates (silica gel 60 F254 grade, $0.25 \mathrm{~mm}$ ) were used. Acme (India) silica gel (100-200 mesh) was used for column chromatography. 


\section{General procedure 1: Procedure for acid promoted allene formation}

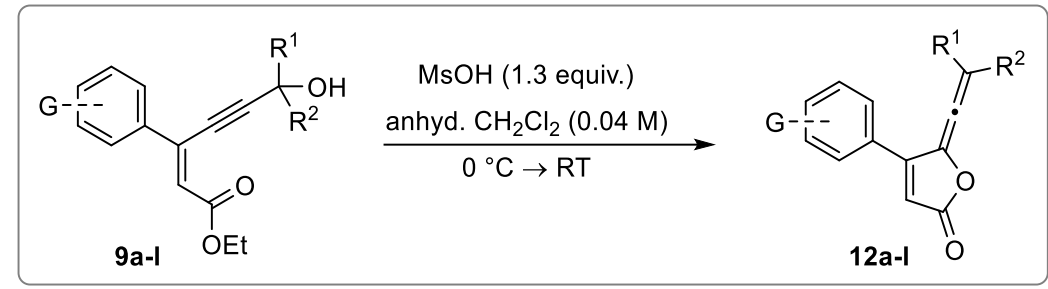

To a well stirred solution of the $(Z)$-enynoate-propargylic alcohol ( 1 equiv.) in anhydrous dichloromethane $(0.04 \mathrm{M})$, methanesulfonic acid $(\mathrm{MsOH})$ (1.3 equiv.) was added drop wise at $0{ }^{\circ} \mathrm{C}$ under nitrogen. The mixture was stirred at same temperature until the TLC showed complete consumption of the starting material. After completion, the reaction was quenched by addition of saturated $\mathrm{NaHCO}_{3}$ solution. The aqueous layer was extracted with $\mathrm{CH}_{2} \mathrm{Cl}_{2}$ (3 times). The combined organic layers were dried over anhydrous $\mathrm{Na}_{2} \mathrm{SO}_{4}$, filtered and concentrated in-vacuo at 0 to $5{ }^{\circ} \mathrm{C}$. Quick purification of crude product via a silica gel column chromatography (Hexane:EtOAc) provided the desired allene derivatives 12a-l.

[N.B. The allenes were found to be very sensitive to heat. Thus the column fractions were also concentrated under reduced pressure at 0 to $5{ }^{\circ} \mathrm{C}$ and submitted for NMR analysis immediately after evaporation and then stored at $\left.-20{ }^{\circ} \mathrm{C}\right]$.

\section{5-(cyclohexylidenemethylene)-4-phenylfuran-2(5H)-one (12a)}

According to the General Procedure 1, propargylic alcohol 9a (60 mg, $0.2 \mathrm{mmol}, 1.0$ equiv.), methanesulfonic acid (MsOH) $(0.017 \mathrm{~mL}, 0.26 \mathrm{mmol}, 1.3$ equiv.) were used in $5 \mathrm{~mL}$ anhydrous $\mathrm{CH}_{2} \mathrm{Cl}_{2}$, affording the desired allene 12a as a white crystalline solid (46 mg, $0.18 \mathrm{mmol}, 90 \%$ ) by using Hexane/EtOAc (9:1) as eluent.

${ }^{1} \mathbf{H}$ NMR $\left(400 \mathrm{MHz}, \mathrm{CDCl}_{3}\right): \delta_{\mathrm{H}} 7.58-7.53(\mathrm{~m}, 2 \mathrm{H}), 7.47-7.40(\mathrm{~m}, 3 \mathrm{H})$, $6.25(\mathrm{~s}, 1 \mathrm{H}), 2.39-2.33(\mathrm{~m}, 4 \mathrm{H}), 1.84-1.70(\mathrm{~m}, 3 \mathrm{H})$ and $1.62-1.60(\mathrm{~m}$, 3H) $\mathrm{ppm}$.

${ }^{13} \mathrm{C}$ NMR $\left(100 \mathrm{MHz}, \mathrm{CDCl}_{3}\right): \delta_{\mathrm{C}} 187.1,169.0,156.6,130.9,130.6,128.8$,

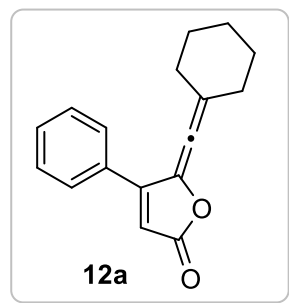
$128.3,128.1,121.3,113.0,32.3,26.9$ and $25.7 \mathrm{ppm}$.

IR (ATR): 3452, 1758, 1200, 1079, 936, 768, 692, 466 and $420 \mathrm{~cm}^{-1}$.

HRMS (ESI) m/z: [M + Na] ${ }^{+}$Calcd for $\mathrm{C}_{17} \mathrm{H}_{16} \mathrm{NaO}_{2} 275.1048$; found 275.1061.

TLC: $\mathrm{R}_{\mathrm{f}}=0.4$ (9:1, Hex/EtOAc).

MP: $86-88{ }^{\circ} \mathrm{C}$ (recrystallized from $\mathrm{CH}_{2} \mathrm{Cl}_{2} /$ Hexane: $1: 1$ ). 


\section{5-((4-methylcyclohexylidene)methylene)-4-phenylfuran-2(5H)-one (12b)}

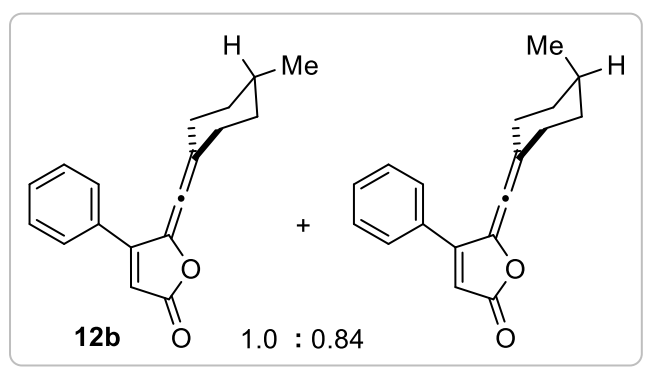

(Inseparable mixture of diastereomers with $d . r .=1.0: 0.84$ ).

According to the General Procedure 1, propargylic alcohol 9b (62 mg, $0.2 \mathrm{mmol}, 1.0$ equiv.), methanesulfonic acid (MsOH) (0.017 mL, $0.26 \mathrm{mmol}, 1.3$ equiv.) were used in $5 \mathrm{~mL}$ anhydrous $\mathrm{CH}_{2} \mathrm{Cl}_{2}$, affording the desired allene $\mathbf{1 2 b}$ as on yellow liquid ( $46 \mathrm{mg}, 0.18 \mathrm{mmol}, 86 \%$ ) by using Hexane/EtOAc (9:1) as eluent.

${ }^{1} \mathbf{H}$ NMR $\left(400 \mathrm{MHz}, \mathrm{CDCl}_{3}\right)$ : Major isomer: $\delta_{\mathrm{H}} 7.63-7.56(1 \mathrm{H}, \mathrm{m}), 7.56-7.49(1 \mathrm{H}, \mathrm{m})$, $7.49-7.35(3 \mathrm{H}, \mathrm{m}), 6.25(1 \mathrm{H}, \mathrm{s}), 2.61-2.46(2 \mathrm{H}, \mathrm{m}), 2.29(\mathrm{td}, J=13.4,4.0 \mathrm{~Hz}, 2 \mathrm{H}), 1.96-$ $1.78(3 \mathrm{H}, \mathrm{m}), 1.35-1.27(1 \mathrm{H}, \mathrm{m}), 1.14-1.02(1 \mathrm{H}, \mathrm{m})$ and $0.94(\mathrm{~d}, J=6.5 \mathrm{~Hz}, 3 \mathrm{H}) \mathrm{ppm}$.

Minor isomer: $\delta_{\mathrm{H}} 7.63-7.56(1 \mathrm{H}, \mathrm{m}), 7.56-7.49(1 \mathrm{H}, \mathrm{m}), 7.49-7.35(3 \mathrm{H}, \mathrm{m}), 6.25(1 \mathrm{H}$, s), $2.61-2.46(2 \mathrm{H}, \mathrm{m}), 2.20(\mathrm{td}, J=13.3,4.1 \mathrm{~Hz}, 2 \mathrm{H}), 1.96-1.78(3 \mathrm{H}, \mathrm{m}), 1.35-1.27(1 \mathrm{H}$, $\mathrm{m}), 1.14-1.02(1 \mathrm{H}, \mathrm{m})$ and $0.97(\mathrm{~d}, J=6.5 \mathrm{~Hz}, 3 \mathrm{H}) \mathrm{ppm}$.

${ }^{13} \mathrm{C}$ NMR $\left(100 \mathrm{MHz}, \mathrm{CDCl}_{3}\right): \delta_{\mathrm{C}} 187.0,168.9,156.5,130.9,130.8,130.4,130.4,128.8,128.6$, 128.1, 127.9, 125.3, 125.1, 121.0, 120.8, 112.9, 112.7, 35.0, 34.8, 31.9, 31.7, 31.6, 31.5, 21.9 and $21.7 \mathrm{ppm}$.

IR (ATR): 2961, 2936, 1808, 1366, 1288, 1219, 1179, 963, 953, 818, 774, 767, 758 and 736 $\mathrm{cm}^{-1}$.

HRMS (ESI) m/z: [M + Na] ${ }^{+}$Calcd for $\mathrm{C}_{18} \mathrm{H}_{18} \mathrm{NaO}_{2} 289.1204$; found 289.1212.

TLC: $\mathrm{R}_{\mathrm{f}}=0.45(9: 1, \mathrm{Hex} / \mathrm{EtOAc})$. 


\section{5-((4-methoxycyclohexylidene)methylene)-4-phenylfuran-2(5H)-one (12c)}

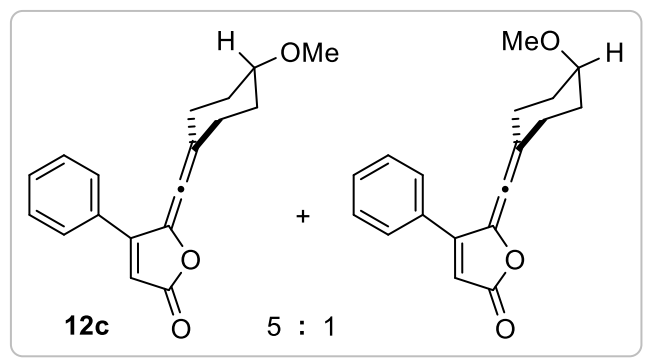

(Inseparable mixture of diastereomers with $d . r .=5: 1$ ).

According to the General Procedure 1, propargylic alcohol 9c (66 mg, $0.2 \mathrm{mmol}, 1.0$ equiv.), methanesulfonic acid (MsOH) $(0.017 \mathrm{~mL}, 0.26 \mathrm{mmol}, 1.3$ equiv.) were used in $5 \mathrm{~mL}$ anhydrous $\mathrm{CH}_{2} \mathrm{Cl}_{2}$, affording the desired allene $12 \mathrm{c}$ as a colourless oil (46 $\mathrm{mg}, 0.16 \mathrm{mmol}, 81 \%$ ) by using Hexane/EtOAc (4:1) as eluent.

${ }^{1} \mathbf{H}$ NMR $\left(400 \mathrm{MHz}, \mathrm{CDCl}_{3}\right)$ : Major isomer: $\delta_{\mathrm{H}} 7.57-7.51(2 \mathrm{H}, \mathrm{m}), 7.50-7.39(3 \mathrm{H}, \mathrm{m})$, $6.26(1 \mathrm{H}, \mathrm{s}), 3.49-3.40(1 \mathrm{H}, \mathrm{m}), 3.37(3 \mathrm{H}, \mathrm{s}), 2.67-2.57(\mathrm{~m}, 2 \mathrm{H}), 2.31-2.23(2 \mathrm{H}, \mathrm{m}), 1.91$ $-1.77(3 \mathrm{H}, \mathrm{m})$ and $1.74-1.70(1 \mathrm{H}, \mathrm{m}) \mathrm{ppm}$.

Minor isomer: $\delta_{\mathrm{H}} 7.57-7.51(2 \mathrm{H}, \mathrm{m}), 7.50-7.39(3 \mathrm{H}, \mathrm{m}), 6.26(1 \mathrm{H}, \mathrm{s}), 3.49-3.40(1 \mathrm{H}, \mathrm{m})$, $3.37(3 \mathrm{H}, \mathrm{s}), 2.57-2.52(\mathrm{~m}, 2 \mathrm{H}), 2.37-2.31(2 \mathrm{H}, \mathrm{m}), 1.91-1.77(3 \mathrm{H}, \mathrm{m})$ and $1.74-1.70$ $(1 \mathrm{H}, \mathrm{m}) \mathrm{ppm}$.

${ }^{13} \mathrm{C}$ NMR (100 MHz, $\left.\mathrm{CDCl}_{3}\right)$ : Major isomer: $\delta_{\mathrm{C}} 187.1,168.8,156.55,130.9,130.5,128.8$, $128.03,125.4,120.1,113.1,75.4,56.0,30.6$ and $28.0 \mathrm{ppm}$.

Minor isomer: $\delta_{C} 187.1,168.8,156.48,131.0,130.3,128.9,128.00,125.4,119.8,113.0,75.9$, $56.1,30.8$ and $28.4 \mathrm{ppm}$.

IR (ATR): 2944, 1759, 1451, 1277, 1263, 1203, 1100, 1077, 940, 912, 766 and $749 \mathrm{~cm}^{-1}$. HRMS (ESI) m/z: [M + H] $]^{+}$Calcd for $\mathrm{C}_{18} \mathrm{H}_{19} \mathrm{O}_{3}$ 283.1329; found 283.1341.

TLC: $\mathrm{R}_{\mathrm{f}}=0.25$ (9:1, Hex/EtOAc). 


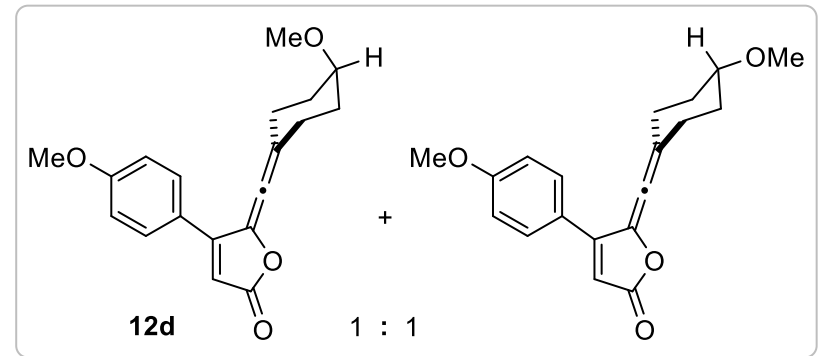

(Inseparable mixture of diastereomers with $d . r .=1: 1$ )

According to the General Procedure 1, propargylic alcohol 9d (72 mg, $0.2 \mathrm{mmol}, 1.0$ equiv.), methanesulfonic acid (MsOH) $(0.017 \mathrm{~mL}, 0.26 \mathrm{mmol}, 1.3$ equiv.) were used in $5 \mathrm{~mL}$ anhydrous $\mathrm{CH}_{2} \mathrm{Cl}_{2}$, affording the desired allene $\mathbf{1 2 d}$ as a colourless oil (54 $\mathrm{mg}, 0.17 \mathrm{mmol}, 86 \%$ ) by using Hexane/EtOAc (2:1) as eluent.

${ }^{1}$ H NMR (400 MHz, $\left.\mathrm{CDCl}_{3}\right): \delta_{\mathrm{H}} 7.53(\mathrm{~d}, J=8.1,4 \mathrm{H}), 6.93(\mathrm{~d}, J=8.0,4 \mathrm{H}), 6.17(\mathrm{~s}, 2 \mathrm{H}), 3.86$ $(\mathrm{s}, 6 \mathrm{H}), 3.50-3.42(\mathrm{~m}, 2 \mathrm{H}), 3.38(\mathrm{~s}, 3 \mathrm{H}), 3.37(\mathrm{~s}, 3 \mathrm{H}), 2.69-2.52(\mathrm{~m}, 4 \mathrm{H}), 2.37-2.24(\mathrm{~m}$, $4 \mathrm{H}), 1.89-1.82(\mathrm{~m}, 4 \mathrm{H})$ and $1.82-1.70(\mathrm{~m}, 4 \mathrm{H}) \mathrm{ppm}$.

${ }^{13} \mathrm{C}$ NMR $\left(100 \mathrm{MHz}, \mathrm{CDCl}_{3}\right): \delta_{\mathrm{C}} 187.1,169.2,169.1,161.9,161.8,155.9,155.8,129.5,125.6$, $125.4,122.9,122.8,119.8,119.5,114.3,114.2$, 111.3, 111.1, 75.9, 75.5, 56.1, 56.0, 55.5, 30.8, 30.6, 28.3 and $28.1 \mathrm{ppm}$.

IR (ATR): 2952, 1761, 1449, 1279, 1242, 1119, 1105, 1060, 952, 920, 771 and $755 \mathrm{~cm}^{-1}$.

HRMS (ESI) m/z: [M + H] ${ }^{+}$Calcd for $\mathrm{C}_{19} \mathrm{H}_{21} \mathrm{O}_{4}$ 313.1434; found 313.1430.

TLC: $\mathrm{R}_{\mathrm{f}}=0.25(9: 1, \mathrm{Hex} / \mathrm{EtOAc})$.

\section{5-(cycloheptylidenemethylene)-4-phenylfuran-2(5H)-one (12e)}

According to the General Procedure 1, propargylic alcohol 9e (62 mg, $0.2 \mathrm{mmol}, 1.0$ equiv.), methanesulfonic acid $(\mathrm{MsOH})(0.017 \mathrm{~mL}, 0.26 \mathrm{mmol}, 1.3$ equiv.) were used in $5 \mathrm{~mL}$ dry $\mathrm{CH}_{2} \mathrm{Cl}_{2}$, affording the desired allene $\mathbf{1 2 e}$ as a pale yellow oil (46 $\mathrm{mg}, 0.17 \mathrm{mmol}, 87 \%$ ) by using Hexane/EtOAc (9:1) as eluent.

${ }^{1} \mathbf{H}$ NMR $\left(400 \mathrm{MHz}, \mathrm{CDCl}_{3}\right): \delta_{\mathrm{H}} 7.62-7.50(2 \mathrm{H}, \mathrm{m}), 7.49-7.37(3 \mathrm{H}, \mathrm{m}), 6.25(1 \mathrm{H}, \mathrm{s}), 2.59$ $-2.43(4 \mathrm{H}, \mathrm{m}), 1.80-1.71(2 \mathrm{H}, \mathrm{m})$ and $1.67-1.54(6 \mathrm{H}, \mathrm{m}) \mathrm{ppm}$. 
${ }^{13}$ C NMR (100 MHz, $\left.\mathrm{CDCl}_{3}\right): \delta_{\mathrm{C}} 190.2,169.1,156.6,130.9,130.5,128.8$, $128.0,125.3,124.0,112.9,33.4,29.4$ and $27.7 \mathrm{ppm}$.

HRMS (ESI) m/z: $[\mathrm{M}+\mathrm{Na}]^{+}$Calcd for $\mathrm{C}_{18} \mathrm{H}_{18} \mathrm{NaO}_{2}$ 289.1199; found 289.1207.

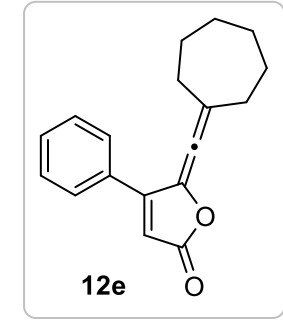

TLC: $\mathrm{R}_{\mathrm{f}}=0.45(9: 1, \mathrm{Hex} / \mathrm{EtOAc})$.

\section{5-(cyclooctylidenemethylene)-4-(4-methoxyphenyl)furan-2(5H)-one (12f)}

According to the General Procedure 1, propargylic alcohol 9f (71 mg, $0.2 \mathrm{mmol}, 1.0$ equiv.), methanesulfonic acid (MsOH) (0.017 mL, $0.26 \mathrm{mmol}, 1.3$ equiv.) were used in $5 \mathrm{~mL}$ anhydrous $\mathrm{CH}_{2} \mathrm{Cl}_{2}$, affording the desired allene $12 \mathrm{f}$ as colourless gummy liquid (47 $\mathrm{mg}, 0.15 \mathrm{mmol}, 75 \%$ ) by using Hexane/EtOAc (4:1) as eluent.

${ }^{1} \mathrm{H}$ NMR $\left(400 \mathrm{MHz}, \mathrm{CDCl}_{3}\right): \delta_{\mathrm{H}} 7.52(\mathrm{~d}, J=8.0,2 \mathrm{H}), 6.92(\mathrm{~d}, J=8.0$, 2H), $6.17(\mathrm{~s}, 1 \mathrm{H}), 3.85(\mathrm{~s}, 3 \mathrm{H}), 2.53-2.44(\mathrm{~m}, 2 \mathrm{H}), 2.42-2.34(\mathrm{~m}$, $2 \mathrm{H}), 1.83-1.70(\mathrm{~m}, 4 \mathrm{H})$ and $1.65-1.48(\mathrm{~m}, 6 \mathrm{H}) \mathrm{ppm}$.

${ }^{13} \mathrm{C}$ NMR $\left(100 \mathrm{MHz}, \mathrm{CDCl}_{3}\right): \delta_{\mathrm{C}} 190.3,169.5,161.8,156.0,129.5$, $126.4,124.8,123.0,114.3,111.2,55.5,32.6,26.9,26.8$ and $25.6 \mathrm{ppm}$.

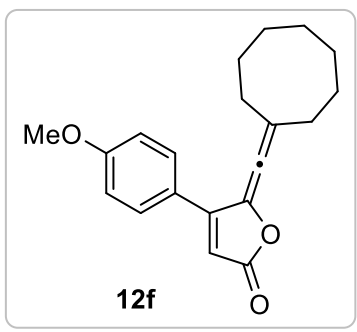

IR (ATR): 3654, 2925, 2852, 1751, 1604, 1509, 1455, 1257, 1180, 1074, 1029, 953 and 920 $\mathrm{cm}^{-1}$.

HRMS (ESI) m/z: [M + H] ${ }^{+}$Calcd for $\mathrm{C}_{20} \mathrm{H}_{23} \mathrm{O}_{3} 311.1642$; found 311.1636.

TLC: $\mathrm{R}_{\mathrm{f}}=0.45(4: 1, \mathrm{Hex} / \mathrm{EtOAc})$.

\section{5-(cyclooctylidenemethylene)-4-phenylfuran-2(5H)-one (12g)}

According to the General Procedure 1, propargylic alcohol 9g (65 mg, $0.2 \mathrm{mmol}, 1.0$ equiv.), methanesulfonic acid $(\mathrm{MsOH})(0.017 \mathrm{~mL}, 0.26 \mathrm{mmol}, 1.3$ equiv. $)$ were used in $5 \mathrm{~mL}$ anhydrous $\mathrm{CH}_{2} \mathrm{Cl}_{2}$, affording the desired allene $\mathbf{1 2 g}$ as colourless gummy liquid (40 $\mathrm{mg}, 0.14 \mathrm{mmol}, 71 \%$ ) by using Hexane/EtOAc $(9: 1)$ as eluent.

${ }^{1} \mathbf{H}$ NMR $\left(400 \mathrm{MHz}, \mathrm{CDCl}_{3}\right): \delta_{\mathrm{H}} 7.58-7.50(2 \mathrm{H}, \mathrm{m}), 7.48-7.38(3 \mathrm{H}, \mathrm{m})$, $6.25(1 \mathrm{H}, \mathrm{s}), 2.52-2.43(2 \mathrm{H}, \mathrm{m}), 2.42-2.33(2 \mathrm{H}, \mathrm{m}), 1.78-1.69(4 \mathrm{H}, \mathrm{m})$ and $1.64-1.46(6 \mathrm{H}, \mathrm{m}) \mathrm{ppm}$.

${ }^{13}$ C NMR (100 MHz, $\left.\mathrm{CDCl}_{3}\right): \delta_{\mathrm{C}} 190.3,169.2,156.6,130.8,130.6,128.9$, $128.0,126.4,125.1,113.1,32.6,26.8$ and $25.6 \mathrm{ppm}$.

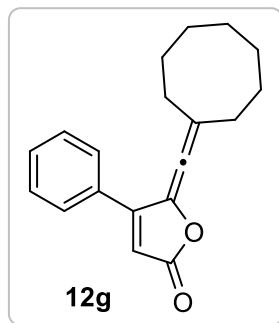

HRMS (ESI) m/z: [M + Na] ${ }^{+}$Calcd for $\mathrm{C}_{19} \mathrm{H}_{20} \mathrm{NaO}_{2}$ 303.1361; found 303.1368. 
TLC: $\mathrm{R}_{\mathrm{f}}=0.6(9: 1, \mathrm{Hex} /$ EtOAc $)$.

\section{Procedure 2: Procedure for ${ }^{18} \mathrm{O}$ labelling experiment.}

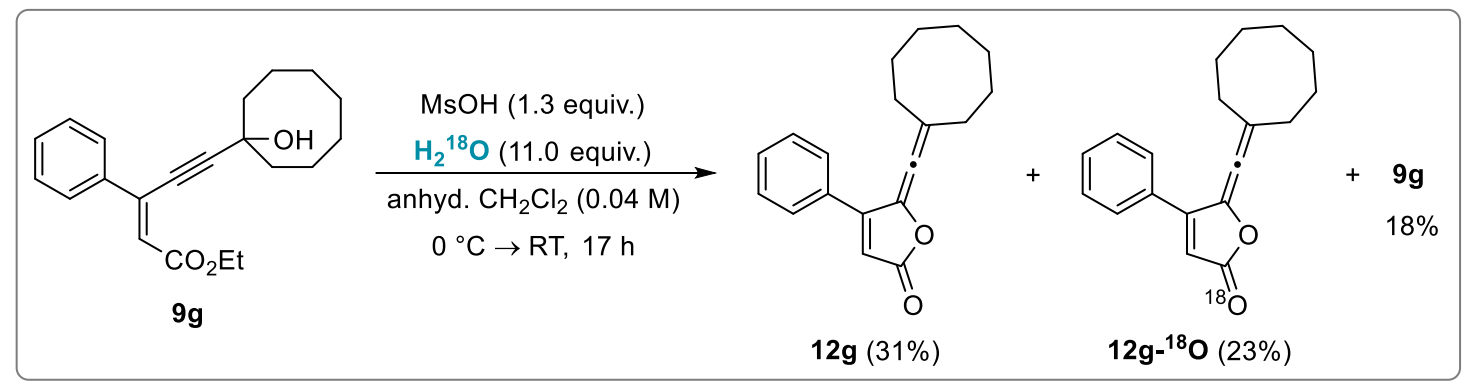

To a well stirred solution of the ethyl (Z)-5-(1-hydroxycyclooctyl)-3-phenylpent-2-en-4-ynoate 9g (65 mg, $0.2 \mathrm{mmol}, 1.0$ equiv.) in $5 \mathrm{~mL}$ anhydrous dichloromethane and $\mathrm{H}_{2}{ }^{18} \mathrm{O}$ (40 $\mu \mathrm{L}, 11.0$ equiv.) methanesulfonic acid ( $\mathrm{MsOH})\left(1.3\right.$ equiv.) was added drop wise at $0{ }^{\circ} \mathrm{C}$ under nitrogen. The mixture was stirred at same temperature until the TLC showed complete consumption of the starting material. After completion, the reaction was quenched by addition of saturated $\mathrm{NaHCO}_{3}$ solution. The aqueous layer was extracted with $\mathrm{CH}_{2} \mathrm{Cl}_{2}$ (3 times). The combined organic layers were dried over anhydrous $\mathrm{Na}_{2} \mathrm{SO}_{4}$, filtered and concentrated in-vacuo at 0 to 5 ${ }^{\circ} \mathrm{C}$. Quick purification of crude product via silica gel column chromatography (Hexane:EtOAc 9:1) provided an inseparable mixture of 5-(cyclooctylidenemethylene)-4-phenylfuran-2(5H)one $\mathbf{1 2 g}$ (17 mg, $0.06 \mathrm{mmol}, 31 \%)$ and its ${ }^{18} \mathrm{O}$ analogue $\mathbf{1 2 g -}{ }^{\mathbf{1 8}} \mathbf{O}$ (13 $\left.\mathrm{mg}, 0.05 \mathrm{mmol}, 23 \%\right)$ in 4:3 ratio. ${ }^{1}$ High resolution positive ion electrospray mass spectra (HRMS-ESI) for the final products were shown.

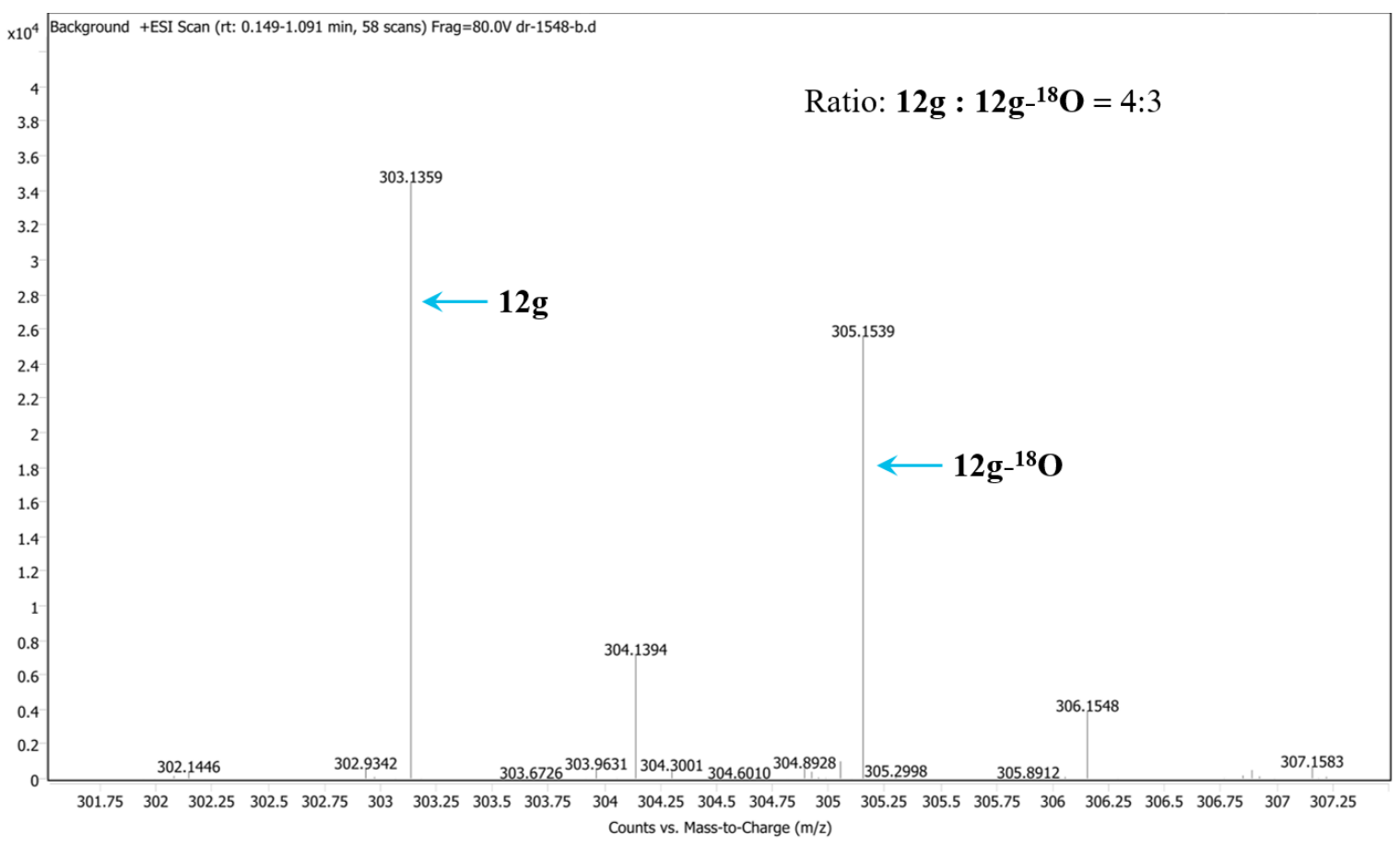


5-(cyclooctylidenemethylene)-4-phenylfuran-2(5H)-one-- ${ }^{18} \mathrm{O}\left(12 \mathrm{~g}-{ }^{18} \mathrm{O}\right)$

${ }^{1} \mathbf{H}$ NMR (400 MHz, $\left.\mathrm{CDCl}_{3}\right): \delta_{\mathrm{H}} 7.59-7.50(\mathrm{~m}, 2 \mathrm{H}), 7.49-7.37$ (m, 3H), $6.25(\mathrm{~s}, 1 \mathrm{H}), 2.52-2.43(\mathrm{~m}, 2 \mathrm{H}), 2.42-2.34(\mathrm{~m}, 2 \mathrm{H}), 1.77-1.68(\mathrm{~m}, 5 \mathrm{H})$ and $1.65-1.48(\mathrm{~m}, 5 \mathrm{H}) \mathrm{ppm}$.

${ }^{13}$ C NMR $\left(100 \mathrm{MHz}, \mathrm{CDCl}_{3}\right): \delta_{\mathrm{C}} 190.3,169.2,156.7,130.8,130.6,128.9$, 128.0, 126.4, 125.1, 113.1, 32.6, 26.8 and $25.6 \mathrm{ppm}$.

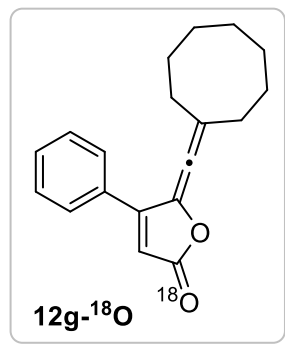

HRMS (ESI) m/z: [M + Na] ${ }^{+}$Calcd for $\mathrm{C}_{19} \mathrm{H}_{21} \mathrm{Na}^{18} \mathrm{O}^{16} \mathrm{O} 305.1398$; found 305.1539.

TLC: $R_{\mathrm{f}}=0.6(9: 1, \mathrm{Hex} / \mathrm{EtOAc})$.

\section{5-(2-methylprop-1-en-1-ylidene)-4-phenylfuran-2(5H)-one (12h)}

According to the General Procedure 1, propargylic alcohol 9h (52 mg, $0.2 \mathrm{mmol}, 1.0$ equiv.), methanesulfonic acid (MsOH) $(0.017 \mathrm{~mL}, 0.26 \mathrm{mmol}, 1.3$ equiv.) were used in $5 \mathrm{~mL}$ anhydrous $\mathrm{CH}_{2} \mathrm{Cl}_{2}$, affording the desired allene $\mathbf{1 2 h}$ as a colourless oil (34 $\mathrm{mg}, 0.16 \mathrm{mmol}, 78 \%$ ) by using Hexane/EtOAc (9:1) as eluent.

${ }^{1} \mathbf{H}$ NMR $\left(400 \mathrm{MHz}, \mathrm{CDCl}_{3}\right): \delta_{\mathrm{H}} 7.59-7.50(2 \mathrm{H}, \mathrm{m}), 7.50-7.38(3 \mathrm{H}, \mathrm{m})$, $6.26(1 \mathrm{H}, \mathrm{s})$ and $1.98(6 \mathrm{H}, \mathrm{s}) \mathrm{ppm}$.

${ }^{13}$ C NMR (100 MHz, $\left.\mathrm{CDCl}_{3}\right): \delta_{\mathrm{C}} 190.3,168.9,156.5,130.9,130.5,128.9$, $127.9,125.4,115.2,113.3$ and $21.6 \mathrm{ppm}$.

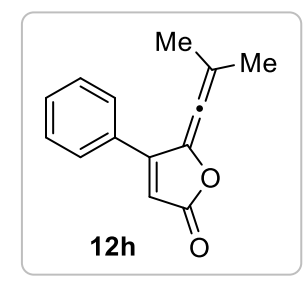

HRMS (ESI) m/z: [M + Na $]^{+}$Calcd for $\mathrm{C}_{14} \mathrm{H}_{12} \mathrm{NaO}_{2}$ 235.0730; found 235.0732.

TLC: $\mathrm{R}_{\mathrm{f}}=0.4(9: 1, \mathrm{Hex} / \mathrm{EtOAc})$.

\section{5-(2,3-dimethylbut-1-en-1-ylidene)-4-phenylfuran-2(5H)-one (12i)}

According to the General Procedure 1, propargylic alcohol 9i (57 mg, $0.2 \mathrm{mmol}, 1.0$ equiv.), methanesulfonic acid (MsOH) $(0.017 \mathrm{~mL}, 0.26 \mathrm{mmol}, 1.3$ equiv.) were used in $5 \mathrm{~mL}$ anhydrous $\mathrm{CH}_{2} \mathrm{Cl}_{2}$, affording the desired allene 12i as a colourless oil (44 $\mathrm{mg}, 0.18 \mathrm{mmol}, 91 \%$ ) by using Hexane/EtOAc (9:1) as eluent.

${ }^{1} \mathbf{H}$ NMR $\left(400 \mathrm{MHz}, \mathrm{CDCl}_{3}\right): \delta_{\mathrm{H}} 7.60-7.51(2 \mathrm{H}, \mathrm{m}), 7.49-7.39(3 \mathrm{H}, \mathrm{m})$, $6.27(1 \mathrm{H}, \mathrm{s}), 2.47-2.34(1 \mathrm{H}, \mathrm{m}), 1.99(3 \mathrm{H}, \mathrm{s}) 1.10(3 \mathrm{H}, \mathrm{d}, J=8.0 \mathrm{~Hz})$ and $1.05(3 \mathrm{H}, \mathrm{d}, J=8.0 \mathrm{~Hz}) \mathrm{ppm}$.

${ }^{13}$ C NMR (100 MHz, $\left.\mathrm{CDCl}_{3}\right): \delta_{\mathrm{C}} 189.2,169.0,156.4,130.8,130.4,128.8$,

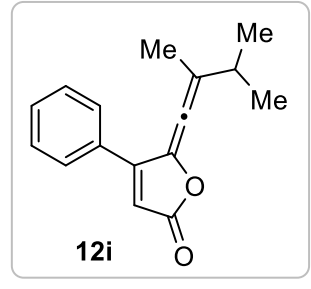
$127.9,127.6,125.2,113.0,34.2,21.1,20.8$ and $18.2 \mathrm{ppm}$. 
HRMS (ESI) m/z: [M + Na] $]^{+}$Calcd for $\mathrm{C}_{16} \mathrm{H}_{16} \mathrm{NaO}_{2}$ 263.1043; found 263.1047.

TLC: $\mathrm{R}_{\mathrm{f}}=0.45(9: 1, \mathrm{Hex} / \mathrm{EtOAc})$.

\section{5-(2,4-dimethylpent-1-en-1-ylidene)-4-phenylfuran-2(5H)-one (12j)}

According to the General Procedure 1, propargylic alcohol 9j (60 mg, $0.2 \mathrm{mmol}, 1.0$ equiv.), methanesulfonic acid (MsOH) (0.017 mL, $0.26 \mathrm{mmol}, 1.3$ equiv.) were used in $5 \mathrm{~mL}$ anhydrous $\mathrm{CH}_{2} \mathrm{Cl}_{2}$, affording the desired allene $\mathbf{1 2} \mathbf{j}$ as a colourless oil (46 $\mathrm{mg}, 0.18 \mathrm{mmol}, 89 \%$ ) by using Hexane/EtOAc (9:1) as eluent.

${ }^{1} \mathbf{H}$ NMR $\left(400 \mathrm{MHz}, \mathrm{CDCl}_{3}\right): \delta_{\mathrm{H}} 7.56-7.50(2 \mathrm{H}, \mathrm{m}), 7.48-7.38(3 \mathrm{H}$, m), $6.25(1 \mathrm{H}, \mathrm{s}), 2.10(2 \mathrm{H}, \mathrm{d}, J=7.1 \mathrm{~Hz}), 1.97(3 \mathrm{H}, \mathrm{s}), 1.84-1.75(1 \mathrm{H}$, $\mathrm{m})$ and $0.86(6 \mathrm{H}, \mathrm{d}, J=5.9 \mathrm{~Hz}) \mathrm{ppm}$.

${ }^{13}$ C NMR (100 MHz, $\left.\mathrm{CDCl}_{3}\right): \delta_{\mathrm{C}} 190.6,169.0,156.5,130.9,130.5$,

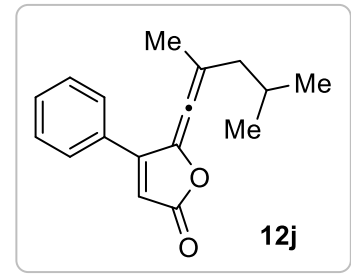
$128.9,128.0,126.4,118.4,113.1,44.7,26.6,22.5,22.5$ and $20.3 \mathrm{ppm}$.

HRMS (ESI) m/z: [M + H] ${ }^{+}$Calcd for $\mathrm{C}_{17} \mathrm{H}_{19} \mathrm{O}_{2}$ 255.1385; found 255.1404.

TLC: $\mathrm{R}_{\mathrm{f}}=0.5(9: 1, \mathrm{Hex} / \mathrm{EtOAc})$.

\section{5-(2-ethylhex-1-en-1-ylidene)-4-phenylfuran-2(5H)-one (12k)}

According to the General Procedure 1, propargylic alcohol 9k (63 mg, $0.2 \mathrm{mmol}, 1.0$ equiv.), methanesulfonic acid (MsOH) $(0.017 \mathrm{~mL}, 0.26 \mathrm{mmol}, 1.3$ equiv.) were used in $5 \mathrm{~mL}$ anhydrous $\mathrm{CH}_{2} \mathrm{Cl}_{2}$, affording the desired allene $12 \mathbf{k}$ as a colourless oil (47 $\mathrm{mg}, 0.18 \mathrm{mmol}, 88 \%$ ) by using Hexane/EtOAc (6:1) as eluent.

${ }^{1} \mathbf{H}$ NMR $\left(400 \mathrm{MHz}, \mathrm{CDCl}_{3}\right): \delta_{\mathrm{H}} 7.58-7.53(2 \mathrm{H}, \mathrm{m}), 7.50-7.37(3 \mathrm{H}$, m), $6.27(1 \mathrm{H}, \mathrm{s}), 2.32-2.17(\mathrm{~m}, 4 \mathrm{H}), 1.44(\mathrm{dq}, J=14.7,7.5,2 \mathrm{H}), 1.29$ $(\mathrm{dt}, J=20.9,7.1,3 \mathrm{H}), 1.07(\mathrm{t}, J=7.5,3 \mathrm{H})$ and $0.84(\mathrm{t}, J=7.3,3 \mathrm{H}) \mathrm{ppm}$. ${ }^{13}$ C NMR $\left(100 \mathrm{MHz}, \mathrm{CDCl}_{3}\right): \delta_{\mathrm{C}} 189.5,169.1,156.3,130.8,130.5,129.9$,

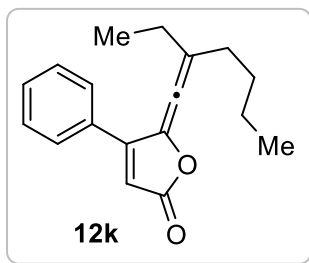
$128.8,127.9,126.2,113.0,33.8,29.5,27.4,22.3,13.9$ and $12.0 \mathrm{ppm}$

IR (ATR): 2961, 2936, 1808, 1366, 1288, 1219, 1179, 963, 953, 818, 774, 767, 758 and 736 $\mathrm{cm}^{-1}$.

HRMS (ESI) m/z: [M + Na] ${ }^{+}$Calcd for $\mathrm{C}_{18} \mathrm{H}_{20} \mathrm{NaO}_{2}$ 291.1356; found 291.1368.

TLC: $\mathrm{R}_{\mathrm{f}}=0.25(9: 1, \mathrm{Hex} / \mathrm{EtOAc})$. 


\section{5-(cyclohexylidenemethylene)-4-(3-nitrophenyl)furan-2(5H)-one (12l)}

According to the General Procedure 1, propargylic alcohol 91 (69 mg, $0.2 \mathrm{mmol}, 1.0$ equiv.), methanesulfonic acid (MsOH) (0.017 mL, $0.26 \mathrm{mmol}, 1.3$ equiv.) were used in $5 \mathrm{~mL}$ anhydrous $\mathrm{CH}_{2} \mathrm{Cl}_{2}$, affording the desired allene $\mathbf{1 2 l}$ as an orange crystalline solid (27 $\mathrm{mg}, 0.09 \mathrm{mmol}$, $46 \%)$ by using Hexane/EtOAc (4:1) as eluent.

${ }^{1}$ H NMR (400 MHz, $\left.\mathrm{CDCl}_{3}\right): \delta_{\mathrm{H}} 8.48(\mathrm{~s}, 1 \mathrm{H}), 8.34(\mathrm{~d}, J=8.2 \mathrm{~Hz}, 1 \mathrm{H}), 7.91$ $(\mathrm{d}, J=7.7 \mathrm{~Hz}, 1 \mathrm{H}), 7.66(\mathrm{t}, J=7.9 \mathrm{~Hz}, 1 \mathrm{H}), 6.40(\mathrm{~s}, 1 \mathrm{H}), 2.45-2.38(\mathrm{~m}$, $4 \mathrm{H})$ and $1.86-1.65(\mathrm{~m}, 6 \mathrm{H}) \mathrm{ppm}$.

${ }^{13} \mathrm{C}$ NMR $\left(100 \mathrm{MHz}, \mathrm{CDCl}_{3}\right): \delta_{\mathrm{C}} 186.6,168.2,153.4,133.9,132.0,130.1$,

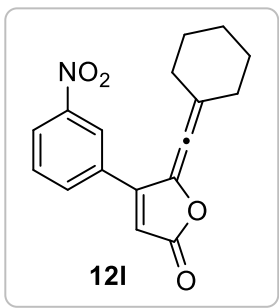
$125.31123 .0,122.8,114.7,32.3,26.8$ and $25.5 \mathrm{ppm}$.

IR (ATR): 3056, 2939, 1757, 1533, 1448, 1352, 1270, 1202, 1075, 987, 923, 755, 740 and 700 $\mathrm{cm}^{-1}$.

HRMS (ESI) m/z: [M + H] $]^{+}$Calcd for $\mathrm{C}_{17} \mathrm{H}_{16} \mathrm{NO}_{4}$ 298.1074; found 298.1070.

TLC: $\mathrm{R}_{\mathrm{f}}=0.2(6: 1, \mathrm{Hex} / \mathrm{EtOAc})$.

MP: $90-92{ }^{\circ} \mathrm{C}$ (recrystallized from $\mathrm{CH}_{2} \mathrm{Cl}_{2}$ / Hexane: $1: 1$ ).

\section{Procedure 3: Acid promoted allene formation in presence of oxygen.}

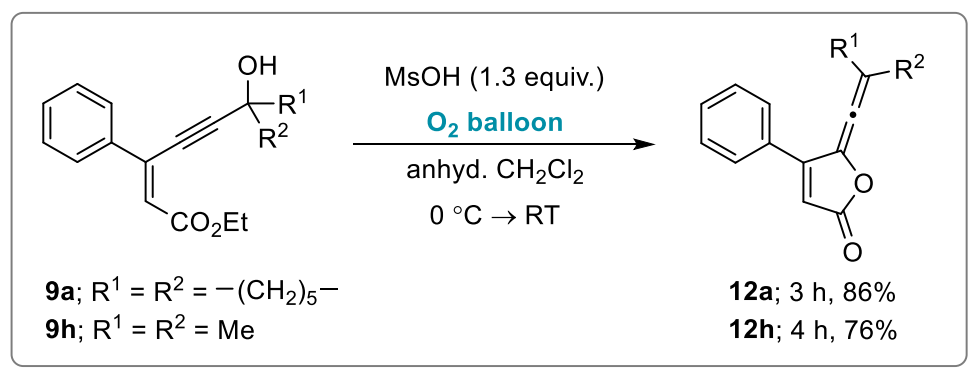

To a well stirred solution of the (Z)-enynoate-propargylic alcohol 9a and $9 \mathbf{h}$ (1 equiv.) in anhydrous dichloromethane $(0.04 \mathrm{M})$, methanesulfonic acid $(\mathrm{MsOH})$ (1.3 equiv.) was added drop wise in a $15 \mathrm{~mL}$ tube with an $\mathrm{O}_{2}$ balloon at $0{ }^{\circ} \mathrm{C}$. The mixture was stirred at same temperature under oxygen atmosphere until the TLC showed complete consumption of the starting material. After completion, the reaction was quenched by addition of saturated $\mathrm{NaHCO}_{3}$ solution. The aqueous layer was extracted with $\mathrm{CH}_{2} \mathrm{Cl}_{2}$ (3 times). The combined organic layers were dried over anhydrous $\mathrm{Na}_{2} \mathrm{SO}_{4}$, filtered and concentrated in-vacuo at 0 to 5 ${ }^{\circ} \mathrm{C}$. Quick purification of crude product via a silica gel column chromatography 
(Hexane:EtOAc) provided the desired allene derivatives 12a $(86 \%)$ and 12h $(76 \%)$ respectively.

\section{General procedure 4: Procedure for acid catalysed isomerization of allenes.}

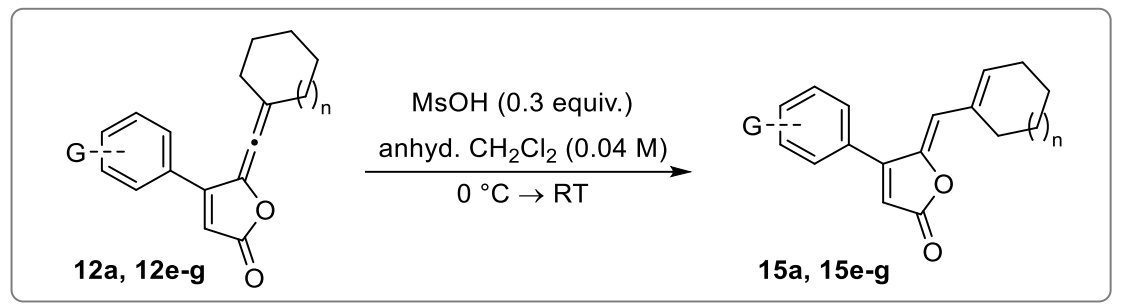

To a solution of the freshly prepared allene (1equiv.) in anhydrous $\mathrm{CH}_{2} \mathrm{Cl}_{2}$, methanesulfonic acid $(\mathrm{MsOH})\left(0.3\right.$ equiv.) was added drop wise at $0{ }^{\circ} \mathrm{C}$ under nitrogen. The reaction mixture was warmed to room temperature and stirred until the TLC showed complete consumption of the allene. The reaction was quenched by addition of saturated $\mathrm{NaHCO}_{3}$ solution. The aqueous layer was extracted with $\mathrm{CH}_{2} \mathrm{Cl}_{2}$ (3 times). The combined organic layers were dried over anhydrous $\mathrm{Na}_{2} \mathrm{SO}_{4}$, filtered and concentrated in-vacuo. Purification of crude product via a silica gel column chromatography (Hexanes/EtOAc) provided desired triene derivatives.

\section{(Z)-5-(cyclohex-1-en-1-ylmethylene)-4-phenylfuran-2(5H)-one (15a)}

According to the General Procedure 4, freshly prepared allene 12a (25 mg, $0.1 \mathrm{mmol}, 1.0$ equiv.), methanesulfonic acid (MsOH) $(0.002 \mathrm{~mL}, 0.03 \mathrm{mmol}, 0.3$ equiv.) were used in $3 \mathrm{~mL}$ anhydrous $\mathrm{CH}_{2} \mathrm{Cl}_{2}$, affording the desired triene 15a as a colourless oil (23 $\mathrm{mg}, 0.093 \mathrm{mmol}$, 93\%) by using Hexane/EtOAc (9:1) as eluent.

${ }^{1} \mathbf{H}$ NMR $\left(400 \mathrm{MHz}, \mathrm{CDCl}_{3}\right): \delta_{\mathrm{H}} 7.54-7.40(5 \mathrm{H}, \mathrm{m}), 6.22-6.12(1 \mathrm{H}$, m), $6.10(1 \mathrm{H}, \mathrm{s}), 5.79(1 \mathrm{H}, \mathrm{s}), 2.66-2.57(2 \mathrm{H}, \mathrm{m}), 2.26-2.16(2 \mathrm{H}, \mathrm{m})$, $1.74-1.66(2 \mathrm{H}, \mathrm{m})$ and $0.92-0.82(2 \mathrm{H}, \mathrm{m}) \mathrm{ppm}$.

${ }^{13}$ C NMR $\left(100 \mathrm{MHz}, \mathrm{CDCl}_{3}\right): \delta_{\mathrm{C}} 169.4,158.8,145.7,138.5,134.8$,

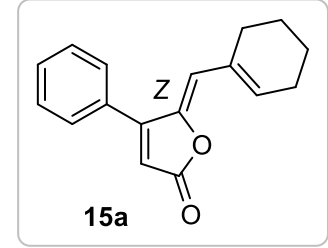
131.0, 130.3, 129.1, 128.6, 118.4, 113.8, 27.4, 27.0, 22.6 and 21.7 ppm.

IR (ATR): 2949, 2361, 1761, 1686, 1519, 1405, 1201, 1141, 1053, 959, 845 and $759 \mathrm{~cm}^{-1}$.

HRMS (ESI) m/z: [M + H] ${ }^{+}$Calcd for $\mathrm{C}_{17} \mathrm{H}_{17} \mathrm{O}_{2}$ 253.1223; found 253.1230.

TLC: $\mathrm{R}_{\mathrm{f}}=0.5(9: 1, \mathrm{Hex} / \mathrm{EtOAc})$. 


\section{(Z)-5-(cyclohept-1-en-1-ylmethylene)-4-phenylfuran-2(5H)-one (15e)}

According to the General Procedure 4, freshly prepared allene 12e (27 mg, $0.1 \mathrm{mmol}, 1.0$ equiv.), methanesulfonic acid ( $\mathrm{MsOH})(0.002 \mathrm{~mL}, 0.03 \mathrm{mmol}, 0.3$ equiv.) were used in $3 \mathrm{~mL}$ anhydrous $\mathrm{CH}_{2} \mathrm{Cl}_{2}$, affording the desired diene $15 \mathbf{e}$ as a colourless oil (24 mg, 0.09 mmol, 90\%) by using Hexane/EtOAc (9:1) as eluent.

${ }^{1} \mathbf{H}$ NMR $\left(400 \mathrm{MHz}, \mathrm{CDCl}_{3}\right): \delta_{\mathrm{H}} 7.52-7.38(\mathrm{~m}, 5 \mathrm{H}), 6.39(\mathrm{t}, J=6.9 \mathrm{~Hz}$, $1 \mathrm{H}), 6.09(\mathrm{~s}, 1 \mathrm{H}), 5.78(\mathrm{~s}, 1 \mathrm{H}), 2.78-2.71(\mathrm{~m}, 2 \mathrm{H}), 2.33-2.35(\mathrm{~m}, 2 \mathrm{H})$, $1.84-1.74(\mathrm{~m}, 2 \mathrm{H})$ and $1.62-1.48(\mathrm{~m}, 4 \mathrm{H}) \mathrm{ppm}$.

${ }^{13} \mathrm{C}$ NMR $\left(100 \mathrm{MHz}, \mathrm{CDCl}_{3}\right): \delta_{\mathrm{C}} 169.3,159.0,145.3,144.0,141.2$,

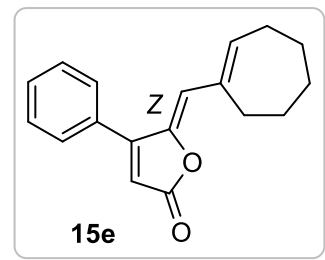
$130.9,130.2,129.0,128.6,119.2,113.5,32.3,30.4,29.5,26.4$ and 26.3 ppm.

IR (ATR): $3155,3005,2361,1785,1683,1523,1402,1201,1148,1046,850$ and $721 \mathrm{~cm}^{-1}$. HRMS (ESI) m/z: [M + H] ${ }^{+}$Calcd for $\mathrm{C}_{18} \mathrm{H}_{19} \mathrm{O}_{2}$ 267.1380; found 267.1382.

TLC: $\mathrm{R}_{\mathrm{f}}=0.5(9: 1, \mathrm{Hex} / \mathrm{EtOAc})$.

\section{(Z)-5-(((E)-cyclooct-1-en-1-yl)methylene)-4-(4-methoxyphenyl)furan-2(5H)-one (15f)}

According to the General Procedure 4, freshly prepared allene $\mathbf{1 2 f}$ (31 mg, $0.1 \mathrm{mmol}, 1.0$ equiv.), methanesulfonic acid (MsOH) (0.002 mL, $0.027 \mathrm{mmol}, 0.3$ equiv.) were used in $3 \mathrm{~mL}$ anhydrous $\mathrm{CH}_{2} \mathrm{Cl}_{2}$, affording the desired triene $\mathbf{1 5 f}$ as a yellow oil (30 $\mathrm{mg}, 0.096 \mathrm{mmol}, 96 \%$ ) by using Hexane/EtOAc (9:1) as eluent.

${ }^{1} \mathbf{H}$ NMR $\left(400 \mathrm{MHz}, \mathrm{CDCl}_{3}\right): \delta_{\mathrm{H}} 7.39(\mathrm{~d}, J=7.7 \mathrm{~Hz}, 2 \mathrm{H}), 7.00(\mathrm{~d}$, $J=7.8 \mathrm{~Hz}, 2 \mathrm{H}), 6.18(\mathrm{t}, J=8.2 \mathrm{~Hz}, 1 \mathrm{H}), 6.03(\mathrm{~s}, 1 \mathrm{H}), 5.81(\mathrm{~s}, 1 \mathrm{H})$, $3.87(\mathrm{~s}, 3 \mathrm{H}), 2.75-2.65(\mathrm{~m}, 2 \mathrm{H}), 2.33-2.23(\mathrm{~m}, 2 \mathrm{H}), 1.76-1.68$ $(\mathrm{m}, 2 \mathrm{H})$, and $1.56-1.41(\mathrm{~m}, 6 \mathrm{H}) \mathrm{ppm}$.

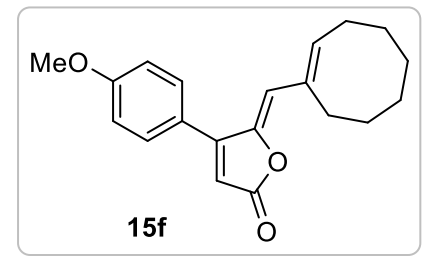

${ }^{13} \mathrm{C}$ NMR $\left(100 \mathrm{MHz}, \mathrm{CDCl}_{3}\right): \delta_{\mathrm{C}} 169.6,161.4,158.6,145.4,142.0,137.6,130.1,123.3,118.1$, $114.5,112.6,55.6,30.0,29.0,28.0,27.1,26.3$ and $25.9 \mathrm{ppm}$.

IR (ATR): 3492, 2923, 2850, 1757, 1605, 1506, 1454, 1341, 1298, 1254, 1179, 1086, 1028, 957,914 and $828 \mathrm{~cm}^{-1}$.

HRMS (ESI) m/z: [M+ H] ${ }^{+}$Calcd for $\mathrm{C}_{20} \mathrm{H}_{23} \mathrm{O}_{3}$ 311.1642; found 311.1641.

TLC: $\mathrm{R}_{\mathrm{f}}=0.7$ (4:1, Hex/EtOAc). 


\section{(Z)-5-(((E)-cyclooct-1-en-1-yl)methylene)-4-phenylfuran-2(5H)-one (15g)}

According to the General Procedure 4, freshly prepared allene $\mathbf{1 2 g}$ ( $28 \mathrm{mg}, 0.1 \mathrm{mmol}, 1.0$ equiv.), methanesulfonic acid ( $\mathrm{MsOH})(0.002 \mathrm{~mL}, 0.03 \mathrm{mmol}, 0.3$ equiv.) were used in $3 \mathrm{~mL}$ anhydrous $\mathrm{CH}_{2} \mathrm{Cl}_{2}$, affording the desired diene $\mathbf{1 5 g}$ as a yellow oil (26 mg, $0.094 \mathrm{mmol}, 94 \%$ ) by using Hexane/EtOAc (9:1) as eluent.

${ }^{1} \mathbf{H}$ NMR $\left(400 \mathrm{MHz}, \mathrm{CDCl}_{3}\right): \delta_{\mathrm{H}} 7.54-7.46(3 \mathrm{H}, \mathrm{m}), 7.46-7.41(2 \mathrm{H}$, m), $6.18(1 \mathrm{H}, \mathrm{t}, J=8.4 \mathrm{~Hz}), 6.10(1 \mathrm{H}, \mathrm{s}), 5.80(1 \mathrm{H}, \mathrm{s}), 2.76-2.65(2 \mathrm{H}$, $\mathrm{m}), 2.31-2.24(2 \mathrm{H}, \mathrm{m}), 1.76-1.70(2 \mathrm{H}, \mathrm{m})$ and $1.56-1.43(6 \mathrm{H}, \mathrm{m})$ ppm.

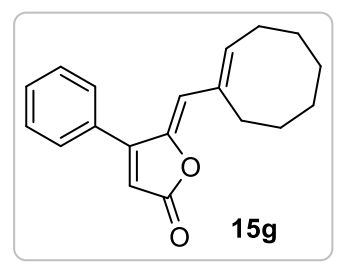

${ }^{13} \mathrm{C}$ NMR $\left(100 \mathrm{MHz}, \mathrm{CDCl}_{3}\right): \delta_{\mathrm{C}} 169.4,158.9,145.3,142.4,137.6,131.0,130.2,129.1,128.7$, 118.0, 113.7, 30.0, 29.0, 28.0, 27.1, 26.2 and $25.9 \mathrm{ppm}$.

IR (ATR): 3680, 3152, 3010, 2361, 1792, 1680, 1520, 1400, 1201, 1150, 1042, 836 and 717 $\mathrm{cm}^{-1}$.

HRMS (ESI) m/z: [M + H] $]^{+}$Calcd for $\mathrm{C}_{19} \mathrm{H}_{21} \mathrm{O}_{2} 281.1536$; found 281.1530.

TLC: $\mathrm{R}_{\mathrm{f}}=0.6(9: 1, \mathrm{Hex} / \mathrm{EtOAc})$.

6. Procedure 5: Procedure for oxidative aromatization of triene 15a.

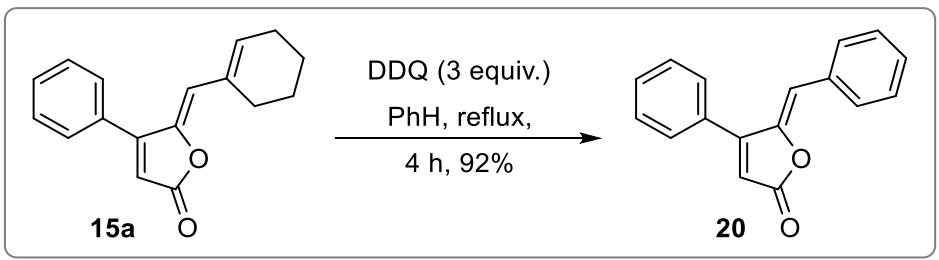

To a well stirred solution of triene $\mathbf{1 5 a}$ (18 $\mathrm{mg}, 0.07 \mathrm{mmol}, 1$ equiv.) in benzene was added DDQ (49 mg, $0.21 \mathrm{mmol}, 3$ equiv.) at room temperature under nitrogen. Reaction mixture was then refluxed in a preheated oil bath for $4 \mathrm{~h}$ after which TLC showed complete consumption of the starting material. Solvent was evaporated in-vacuo. Purification of crude product via a silica gel column chromatography (9:1 Hexanes/EtOAc) provided the desired aromatized product 20 (16 mg, $0.06 \mathrm{mmol}, 92 \%$ yield) as an orange solid. 
${ }^{1} \mathbf{H}$ NMR $\left(400 \mathrm{MHz}, \mathrm{CDCl}_{3}\right): \delta_{\mathrm{H}} 7.85-7.76(\mathrm{~m}, 2 \mathrm{H}), 7.58-7.47(\mathrm{~m}, 5 \mathrm{H})$, $7.43-7.36(\mathrm{~m}, 2 \mathrm{H}), 7.36-7.30(\mathrm{~m}, 1 \mathrm{H}), 6.21(\mathrm{~s}, 1 \mathrm{H})$ and $6.18(\mathrm{~s}, 1 \mathrm{H})$ ppm.

${ }^{13} \mathrm{C}$ NMR (100 MHz, $\left.\mathrm{CDCl}_{3}\right): \delta_{\mathrm{C}} 169.0,158.9,148.1,133.1,130.9,130.6$,

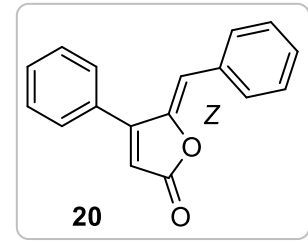
$129.4,129.2,128.9,128.6,114.7$ and $114.0 \mathrm{ppm}$.

IR (ATR): 1757, 1642, 1495, 1449, 1358, 1221, 1181 and $1132 \mathrm{~cm}^{-1}$.

HRMS (ESI) m/z: [M + H] ${ }^{+}$Calcd for $\mathrm{C}_{17} \mathrm{H}_{13} \mathrm{O}_{2}$ 249.0916; found 249.0915.

TLC: $R_{\mathrm{f}}=0.55$ (4:1, Hex/EtOAc).

M.P.: $120-122{ }^{\circ} \mathrm{C}$ (recrystallized from $\mathrm{CH}_{2} \mathrm{Cl}_{2}$ / Hexane: 1:2).

$7 \quad$ General procedure 5: Procedure for synthesis of $\beta$-aryl- $\gamma$-alkylidene- $\gamma$-butenolides derivatives from corresponding propargylic alcohols without isolation of intermediates.

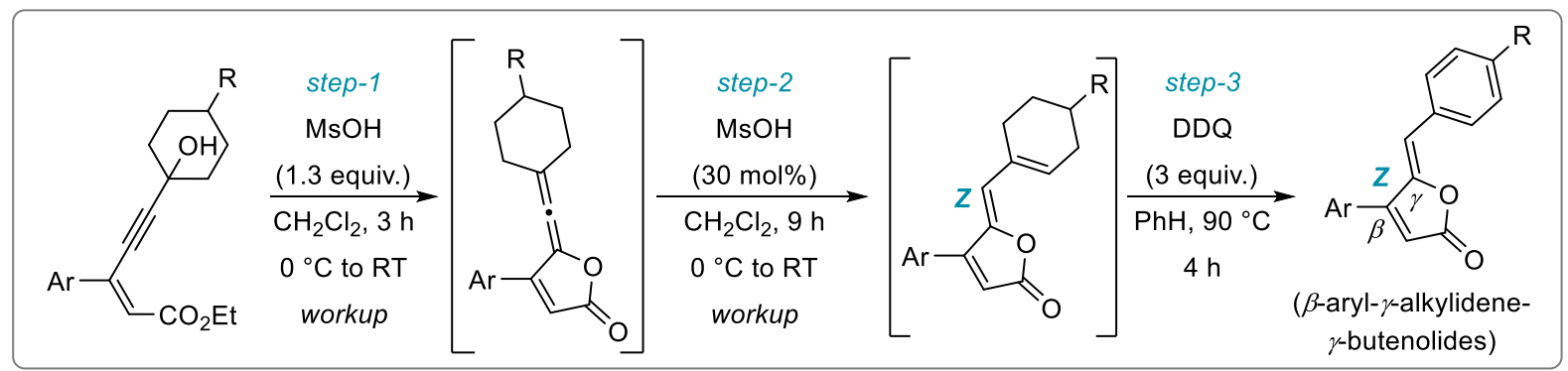

\section{Step 1}

To a well stirred solution of the (Z)-enynoate-propargylic alcohol (1 equiv.) in anhydrous dichloromethane, methanesulfonic acid $(\mathrm{MsOH})$ (1.3 equiv.) was added drop wise at $0{ }^{\circ} \mathrm{C}$ under nitrogen. The mixture was stirred at same temperature for $2.5-3 \mathrm{~h}$. After completion, the reaction was quenched by addition of saturated $\mathrm{NaHCO}_{3}$ solution. The aqueous layer was extracted with $\mathrm{CH}_{2} \mathrm{Cl}_{2}(3 \times 10 \mathrm{~mL})$. The combined organic layers were dried over anhydrous $\mathrm{Na}_{2} \mathrm{SO}_{4}$, filtered and concentrated under reduced pressure at 0 to $5{ }^{\circ} \mathrm{C}$. The crude material was directly used for the next step.

\section{Step 2}

To a solution of the freshly prepared allene (1equiv.) in anhydrous $\mathrm{CH}_{2} \mathrm{Cl}_{2}$, methanesulfonic acid $(\mathrm{MsOH})\left(0.3\right.$ equiv.) was added drop wise at $0{ }^{\circ} \mathrm{C}$ under nitrogen. The reaction mixture was warmed to room temperature and stirred for $9 \mathrm{~h}$. The reaction was quenched by addition of saturated $\mathrm{NaHCO}_{3}$ solution. The aqueous layer was extracted with $\mathrm{CH}_{2} \mathrm{Cl}_{2}(3 \times 10 \mathrm{~mL})$. The 
combined organic layers were dried over anhydrous $\mathrm{Na}_{2} \mathrm{SO}_{4}$, filtered and concentrated in vacuo to give the crude triene which was directly used for the next step.

\section{Step 3}

To a well stirred solution of freshly prepared triene (1 equiv.) in benzene was added DDQ (3 equiv.) at room temperature under nitrogen. Reaction mixture was then refluxed in a preheated oil bath for $4 \mathrm{~h}$. Upon completion solvent was evaporated in-vacuo. Purification of crude product via a silica gel column chromatography (Hexanes/EtOAc) provided the desired triene.

\section{(Z)-5-(4-methylbenzylidene)-4-phenylfuran-2(5H)-one (21)}

According to the General Procedure 5, propargylic alcohol 9b (30 mg, $0.096 \mathrm{mmol}, 1$ equiv.) and methanesulfonic acid (0.008 mL, $0.125 \mathrm{mmol}, 1.3$ equiv.) were used in Step 1 to afford the corresponding allene. The crude allene was used directly with methanesulfonic acid $(0.002$ $\mathrm{mL}, 0.029$ mmol, 0.3 equiv.) in Step 2 to yield the triene 12b. In Step 3, crude triene 12b and DDQ (65 mg, $0.29 \mathrm{mmol}, 3$ equiv.) were used in $4 \mathrm{~mL}$ benzene, affording the desired aromatized product 21 as an orange gummy liquid (17 $\mathrm{mg}, 0.065 \mathrm{mmol}, 68 \%)$ by using Hexane/EtOAc (14:1) as eluent.

${ }^{1} \mathbf{H}$ NMR $\left(400 \mathrm{MHz}, \mathrm{CDCl}_{3}\right): \delta_{\mathrm{H}} 7.71(\mathrm{~d}, J=8.0 \mathrm{~Hz}, 2 \mathrm{H}), 7.58-7.45$ $(\mathrm{m}, 5 \mathrm{H}), 7.20(\mathrm{~d}, J=8.0 \mathrm{~Hz}, 2 \mathrm{H}), 6.19(\mathrm{~s}, 1 \mathrm{H}), 6.17(\mathrm{~s}, 1 \mathrm{H})$ and 2.38 $(\mathrm{s}, 3 \mathrm{H}) \mathrm{ppm}$.

${ }^{13} \mathrm{C}$ NMR $\left(100 \mathrm{MHz}, \mathrm{CDCl}_{3}\right): \delta_{\mathrm{C}} 169.2,158.9,147.5,139.9,131.0$,

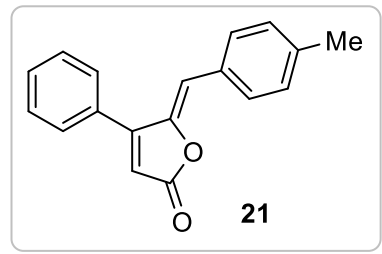
$130.7,130.5,130.4,129.7,129.2,128.7,114.3,114.2$ and $21.6 \mathrm{ppm}$.

IR (ATR): 2924, 1759, 1350, 1223, 1184, 1084, 957, 918, 868, 825, 771 and $698 \mathrm{~cm}^{-1}$.

HRMS (ESI) m/z: [M+ H] ${ }^{+}$Calcd for $\mathrm{C}_{18} \mathrm{H}_{15} \mathrm{O}_{2}$ 263.1067; found 263.1067.

TLC: $\mathrm{R}_{\mathrm{f}}=0.75(4: 1, \mathrm{Hex} / \mathrm{EtOAc})$.

(Z)-5-benzylidene-4-(3-methoxyphenyl)furan-2(5H)-one (22)

According to the General Procedure 5, propargylic alcohol 91 (30 mg, 0.09 mmol, 1 equiv.) and methanesulfonic acid ( $0.008 \mathrm{~mL}, 0.12 \mathrm{mmol}, 1.3$ equiv.) were used in Step 1 to afford the corresponding allene. The crude allene was used directly with methanesulfonic acid $(0.002$ $\mathrm{mL}, 0.03$ mmol, 0.3 equiv.) in Step 2 to yield the corresponding triene. In Step 3, crude triene and DDQ (61 mg, $0.27 \mathrm{mmol}, 3$ equiv.) were used in $4 \mathrm{~mL}$ benzene, affording the desired 
aromatized product 22 as a yellow gummy liquid (18 $\mathrm{mg}, 0.063 \mathrm{mmol}, 70 \%)$ by using Hexane/EtOAc (14:1) as eluent.

${ }^{1} \mathbf{H}$ NMR $\left(400 \mathrm{MHz}, \mathrm{CDCl}_{3}\right): \delta_{\mathrm{H}} 7.80(\mathrm{~d}, J=8.0 \mathrm{~Hz}, 2 \mathrm{H}), 7.47-7.31$ (m, 4H), $7.11-7.04(\mathrm{~m}, 2 \mathrm{H}), 7.02(\mathrm{~s}, 1 \mathrm{H}), 6.21(\mathrm{~s}, 1 \mathrm{H}), 6.20(\mathrm{~s}, 1 \mathrm{H})$ and 3.87 (s, 3H) ppm.

${ }^{13} \mathbf{C}$ NMR $\left(100 \mathrm{MHz}, \mathrm{CDCl}_{3}\right): \delta_{\mathrm{C}} 168.9,160.0,158.8,148.0,133.1$,

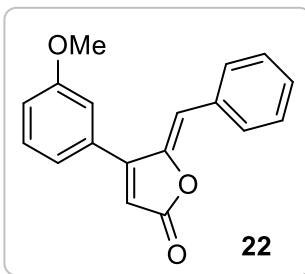
$131.8,131.0,130.3,129.4,128.9,120.9,115.9,114.7,114.4,114.0$ and $55.6 \mathrm{ppm}$.

IR (ATR): 2930, 1763, 1577, 1485, 1454, 1346, 1265, 1211, 1180, 1041, 930, 841, 787 and $694 \mathrm{~cm}^{-1}$.

HRMS (ESI) m/z: [M + H] ${ }^{+}$Calcd for $\mathrm{C}_{18} \mathrm{H}_{15} \mathrm{O}_{3}$ 279.1016; found 279.1019.

TLC: $R_{\mathrm{f}}=0.7$ (4:1, Hex/EtOAc)

(Z)-5-(4-methoxybenzylidene)-4-(4-methoxyphenyl)furan-2(5H)-one (27)

According to the General Procedure 5, propargylic alcohol 9d (30 mg, $0.084 \mathrm{mmol}, 1$ equiv.) and methanesulfonic acid $(0.007 \mathrm{~mL}, 0.11 \mathrm{mmol}, 1.3$ equiv.) were used in Step 1 to afford the allene 12d. The crude allene was used directly with methanesulfonic acid $(0.002 \mathrm{~mL}, 0.025$ mmol, 0.3 equiv.) in Step 2 to yield the corresponding triene. In Step 3, crude triene and DDQ (57 mg, $0.25 \mathrm{mmol}, 3$ equiv.) were used in $4 \mathrm{~mL}$ benzene, affording the desired aromatized product 27 as a yellow solid (18 $\mathrm{mg}, 0.058 \mathrm{mmol}, 69 \%)$ by using Hexane/EtOAc (14:1) as eluent.

${ }^{1} \mathbf{H}$ NMR $\left(400 \mathrm{MHz}, \mathrm{CDCl}_{3}\right): \delta_{\mathrm{H}} 7.77(\mathrm{~d}, J=8.2 \mathrm{~Hz}, 2 \mathrm{H}), 7.46$ $(\mathrm{d}, J=8.0 \mathrm{~Hz}, 2 \mathrm{H}), 7.03(\mathrm{~d}, J=8.0 \mathrm{~Hz}, 2 \mathrm{H}), 6.92(\mathrm{~d}, J=8.2$ $\mathrm{Hz}, 2 \mathrm{H}), 6.16(\mathrm{~s}, 1 \mathrm{H}), 6.09(\mathrm{~s}, 1 \mathrm{H}), 3.89(\mathrm{~s}, 3 \mathrm{H})$ and $3.84(\mathrm{~s}$, 3H) ppm.

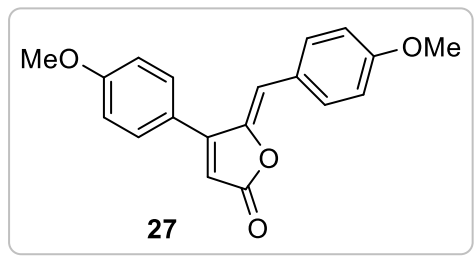

${ }^{13} \mathrm{C}$ NMR $\left(100 \mathrm{MHz}, \mathrm{CDCl}_{3}\right): \delta_{\mathrm{C}} 169.5,161.5,160.6,158.5,146.8,132.7,130.1,126.1,123.1$, $114.7,114.5,113.8,112.4,55.6$ and $55.5 \mathrm{ppm}$.

IR (ATR): 2930, 2852, 1751, 1613, 1515, 1428, 1179, 1090, 1036, 891 and $836 \mathrm{~cm}^{-1}$.

HRMS (ESI) m/z: [M + H] ${ }^{+}$Calcd for $\mathrm{C}_{19} \mathrm{H}_{17} \mathrm{O}_{4}$ 309.1121; found 309.1119.

TLC: $\mathrm{R}_{\mathrm{f}}=0.7(2: 1, \mathrm{Hex} / \mathrm{EtOAc})$.

MP: $134-137{ }^{\circ} \mathrm{C}$. 


\section{Procedure 7: Procedure for deprotection of methyl ether with boron tribromide}

To a stirred solution of $\mathbf{2 6}$ (18 mg, $0.058 \mathrm{mmol}, 1$ equiv.) in anhyd $\mathrm{CH}_{2} \mathrm{Cl}_{2}$ (4 mL) at $-78{ }^{\circ} \mathrm{C}$, was added a $1 \mathrm{M}$ solution of $\mathrm{BBr}_{3}$ in $\mathrm{CH}_{2} \mathrm{Cl}_{2}(0.18 \mathrm{~mL}, 0.18 \mathrm{mmol}, 3$ equiv. $)$ drop wise. The mixture was then allowed to warm up to room temperature and stirred for further $24 \mathrm{~h}$. The reaction was quenched with $\mathrm{H}_{2} \mathrm{O}(5 \mathrm{~mL})$. The organic layer was separated and the aqueous layer was extracted with EtOAc $(3 \times 5 \mathrm{~mL})$. The combined organic layers were washed with $\mathrm{H}_{2} \mathrm{O}$, brine and dried $\left(\mathrm{Na}_{2} \mathrm{SO}_{4}\right)$ and concentrated in vacuo. Purification of crude product via a silica gel column chromatography (3:2 Hexanes/EtOAc) provided the desired Rubrolide E (16 $\mathrm{mg}, 0.056 \mathrm{mmol}, 96 \%$ yield) as a yellow solid.

\section{(Z)-5-(4-hydroxybenzylidene)-4-(4-hydroxyphenyl)furan-2(5H)-one - Rubrolide $\mathrm{E}^{2}$}

${ }^{1}$ H NMR (400 MHz, DMSO-d6): $\delta_{\mathrm{H}} 10.08$ (br s, 1H), 10.01 (br

s, 1H), $7.69(\mathrm{~d}, J=8.0 \mathrm{~Hz}, 2 \mathrm{H}), 7.49$ (d, $J=7.8 \mathrm{~Hz}, 2 \mathrm{H}), 6.93$

$(\mathrm{d}, J=7.8 \mathrm{~Hz}, 2 \mathrm{H}), 6.84(\mathrm{~d}, J=8.0 \mathrm{~Hz}, 2 \mathrm{H}), 6.37(\mathrm{~s}, 1 \mathrm{H})$ and

6.32 (s, 1H) ppm.

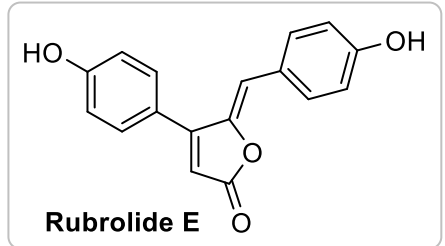

${ }^{13}$ C NMR (100 MHz, DMSO-d6): $\delta_{\mathrm{C}} 168.7,159.7,158.7,158.1,145.3,132.6,130.4,124.2$, $120.7,115.93,115.91,113.4$ and $110.9 \mathrm{ppm}$.

IR (ATR): 3274, 1765, 1727, 1677, 1606, 1520, 1206, 1049 and $746 \mathrm{~cm}^{-1}$.

HRMS (ESI) m/z: [M + H] $]^{+}$Calcd for $\mathrm{C}_{17} \mathrm{H}_{13} \mathrm{O}_{4}$ 281.0808; found 281.0804.

TLC: $\mathrm{R}_{\mathrm{f}}=0.5(1: 1, \mathrm{Hex} / \mathrm{EtOAc})$.

MP: $282-283{ }^{\circ} \mathrm{C}$.

\section{General procedure 8: Procedure for Sonogashira reaction}

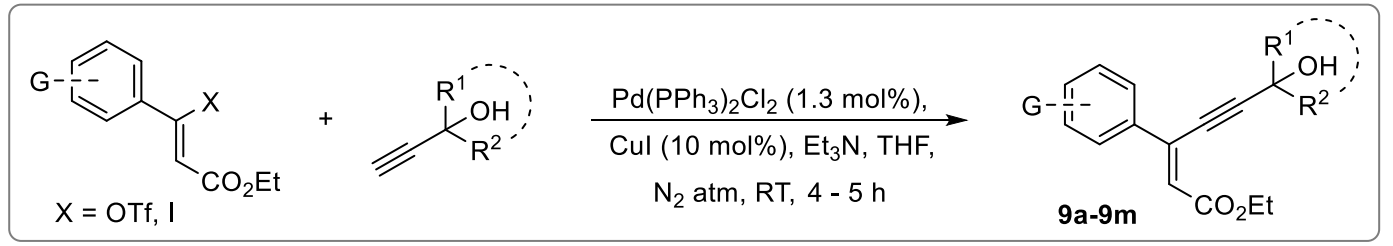

To a solution of the corresponding ( $\mathrm{Z}$ )-enol triflate/iodide (1 equiv.) and the terminal alkyne (1 equiv.) in THF were added $\mathrm{PdCl}_{2}\left(\mathrm{PPh}_{3}\right)_{2}(1.3 \mathrm{~mol} \%)$ and $\mathrm{CuI}(10 \mathrm{~mol} \%)$ followed by addition of triethylamine $(0.5 \mathrm{~mL} / \mathrm{mmol})$. The resulting mixture was stirred at room temperature until the TLC showed complete consumption of the (Z)-enol triflate/iodide. After completion, the reaction was quenched by addition of saturated $\mathrm{NH}_{4} \mathrm{Cl}$ solution. The aqueous layer was 
extracted with EtOAc (3 times). The combined organic layers were dried over anhydrous $\mathrm{Na}_{2} \mathrm{SO}_{4}$, filtered and concentrated in-vacuo. Purification of crude product via a silica gel column chromatography (Hexanes/EA) provided the desired propargyl alcohols 9a-9m.

List of commercially available propargylic alcohols used in Sonogashira reaction

$\underbrace{\substack{\text { 2-methylbut- } \\ \text { 3-yn-2-ol }}}_{\substack{\text { 1-ethynylcyclo } \\ \text { hexan-1-ol }}} \underset{\substack{\text { 3,4-dimethylpent- } \\ \text { 1-yn-3-ol }}}{\mathrm{Me}}$

\section{ethyl (Z)-5-(1-hydroxycyclohexyl)-3-phenylpent-2-en-4-ynoate (9a)}

According to the General Procedure 8, (Z)-enol triflate ${ }^{3}$ (324 mg, 1 mmol, 1 equiv.), 1 ethynylcyclohexan-1-ol (124 mg, $1 \mathrm{mmol}, 1$ equiv.), CuI (19 mg, $0.1 \mathrm{mmol}, 10 \mathrm{~mol} \%)$, $\mathrm{Pd}\left(\mathrm{PPh}_{3}\right)_{2} \mathrm{Cl}_{2}(9 \mathrm{mg}, 0.013 \mathrm{mmol}, 1.3 \mathrm{~mol} \%)$ and $0.5 \mathrm{~mL}$ of triethylamine were used in $15 \mathrm{~mL}$ THF, affording the desired propargylic alcohol 9a as pale yellow oil (280 mg, $0.94 \mathrm{mmol}, 94 \%)$ by using Hexane/EtOAc (4:1) as eluent.

${ }^{1} \mathbf{H}$ NMR $\left(400 \mathrm{MHz}, \mathrm{CDCl}_{3}\right): \delta_{\mathrm{H}} 7.73-7.66(\mathrm{~m}, 2 \mathrm{H}), 7.41-7.35(3 \mathrm{H}$, m), $6.53(\mathrm{~s}, 1 \mathrm{H}), 4.24$ (q, $J=7.1 \mathrm{~Hz}, 2 \mathrm{H}), 3.37$ (br s, 1H), $2.12-2.04$ (m, $2 \mathrm{H}), 1.77-1.52(\mathrm{~m}, 8 \mathrm{H})$ and $1.30(\mathrm{t}, J=7.1 \mathrm{~Hz}, 3 \mathrm{H}) \mathrm{ppm}$.

${ }^{13}$ C NMR (100 MHz, $\left.\mathrm{CDCl}_{3}\right): \delta_{\mathrm{C}} 165.5,137.2,136.4,130.0,128.7,127.2$,

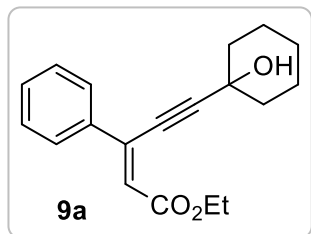
$122.9,107.0,81.5,69.3,60.6,39.8,25.4,23.4$ and $14.4 \mathrm{ppm}$.

IR (ATR): 3431, 2930, 2856, 2370, 2211, 1698, 1450, 1377, 1266, 1168, 1074, 1022, 961, 861 and $770 \mathrm{~cm}^{-1}$.

HRMS (ESI) m/z: [M + H] ${ }^{+}$Calcd for $\mathrm{C}_{19} \mathrm{H}_{22} \mathrm{NaO}_{3} 321.1461$; found 321.1474.

TLC: $\mathrm{R}_{\mathrm{f}}=0.15(9: 1, \mathrm{Hex} / \mathrm{EtOAc})$.

ethyl (Z)-5-(1-hydroxy-4-methylcyclohexyl)-3-phenylpent-2-en-4-ynoate (9b)

According to the General Procedure 8, (Z)-enol triflate ${ }^{3}$ (324 mg, 1 mmol, 1 equiv.), 1 ethynyl-4-methylcyclohexan-1-ol ${ }^{4}$ (138 mg, 1 mmol, 1 equiv.), CuI (19 mg, 0.1 mmol, 10 mol\%), $\mathrm{Pd}\left(\mathrm{PPh}_{3}\right)_{2} \mathrm{Cl}_{2}(9 \mathrm{mg}, 0.013 \mathrm{mmol}, 1.3 \mathrm{~mol} \%)$ and $0.5 \mathrm{~mL}$ of triethylamine were used in $15 \mathrm{~mL}$ THF, affording the desired propargylic alcohol $\mathbf{9 b}$ as a pale yellow oil (275 $\mathrm{mg}, 0.88$ mmol, $88 \%$ ) by using Hexane/EtOAc (4:1) as eluent. 
${ }^{1} \mathbf{H}$ NMR $\left(400 \mathrm{MHz}, \mathrm{CDCl}_{3}\right): \delta_{\mathrm{H}} 7.75-7.65(\mathrm{~m}, 2 \mathrm{H}), 7.44-7.33(\mathrm{~m}$, $3 \mathrm{H}), 6.53$ (s, 1H), 4.23 (q, $J=7.1 \mathrm{~Hz}, 2 \mathrm{H}), 3.36$ (br s, 1H), 2.20-2.09 $(\mathrm{m}, 2 \mathrm{H}), 1.75-1.62(\mathrm{~m}, 4 \mathrm{H}), 1.47-1.35(\mathrm{~m}, 3 \mathrm{H}), 1.29(\mathrm{t}, J=7.1 \mathrm{~Hz}$, $3 \mathrm{H})$ and $0.94(\mathrm{~d}, J=4.9 \mathrm{~Hz}, 3 \mathrm{H}) \mathrm{ppm}$.

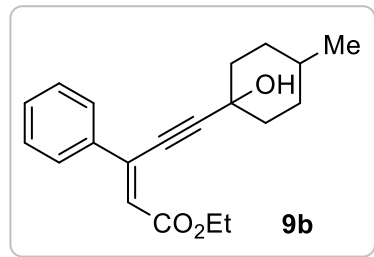

${ }^{13} \mathrm{C}$ NMR (100 MHz, $\left.\mathrm{CDCl}_{3}\right): \delta_{\mathrm{C}} 165.4,137.2,136.4,130.0,128.7,127.3,122.9,106.5,82.1$, $70.1,60.5,39.9,32.5,31.9,21.9$ and $14.4 \mathrm{ppm}$.

IR (ATR): 3437, 2935, 1716, 1600, 1454, 1369, 1269, 1173, 1045, 764 and $694 \mathrm{~cm}^{-1}$.

HRMS (ESI) m/z: [M + H] ${ }^{+}$Calcd for $\mathrm{C}_{20} \mathrm{H}_{25} \mathrm{O}_{3} 313.1798$; found 313.1803.

TLC: $\mathrm{R}_{\mathrm{f}}=0.15(9: 1, \mathrm{Hex} / \mathrm{EtOAc})$.

ethyl (Z)-5-(1-hydroxy-4-methoxycyclohexyl)-3-phenylpent-2-en-4-ynoate (9c)

According to the General Procedure 8, $(Z)$-enol triflate ${ }^{3}$ (324 mg, $1 \mathrm{mmol}, 1$ equiv.), 1 ethynyl-4-methoxycyclohexan-1-ol 26 (154 mg, 1 mmol, 1 equiv.), CuI (19 mg, 0.1 mmol, 10 $\mathrm{mol} \%), \mathrm{Pd}\left(\mathrm{PPh}_{3}\right)_{2} \mathrm{Cl}_{2}(9 \mathrm{mg}, 0.013 \mathrm{mmol}, 1.3 \mathrm{~mol} \%)$ and $0.5 \mathrm{~mL}$ of triethylamine were used in $15 \mathrm{~mL}$ THF, affording the desired propargylic alcohol 9c as a pale yellow oil (289 $\mathrm{mg}, 0.88$ mmol, $88 \%$ ) by using Hexane/EtOAc (4:1) as eluent.

(Inseparable mixture of diastereomers with d.r. 2:1)

${ }^{1} \mathbf{H}$ NMR (400 MHz, $\left.\mathrm{CDCl}_{3}\right)$ : Major isomer: $\delta_{\mathrm{H}} 7.75-7.63(\mathrm{~m}, 2 \mathrm{H})$, 7.44-7.32 (m, 3H), $6.53(1 \mathrm{H}, \mathrm{s}), 4.23$ (q, $J=7.1 \mathrm{~Hz}, 2 \mathrm{H},), 3.40-3.35$ (m, 1H), $3.32(\mathrm{~s}, 3 \mathrm{H}), 2.24-2.15(\mathrm{~m}, 1 \mathrm{H}), 2.11-1.96(\mathrm{~m}, 2 \mathrm{H}), 1.95$ $-1.81(\mathrm{~m}, 4 \mathrm{H}), 1.80-1.68(\mathrm{~m}, 1 \mathrm{H})$ and $1.34-1.27(\mathrm{~m}, 3 \mathrm{H}) \mathrm{ppm}$.

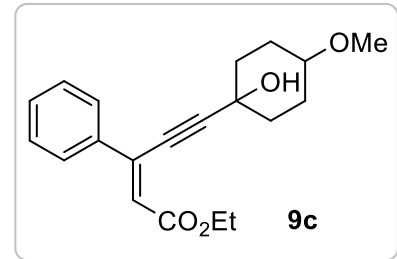

Minor isomer: $\delta_{\mathrm{H}} 7.75-7.63(\mathrm{~m}, 2 \mathrm{H}), 7.44-7.32(\mathrm{~m}, 3 \mathrm{H}), 6.53(\mathrm{~s}, 1 \mathrm{H}), 4.23(\mathrm{q}, J=7.1 \mathrm{~Hz}$, $2 \mathrm{H}), 3.36(\mathrm{~s}, 3 \mathrm{H}), 3.30-3.24(\mathrm{~m}, 1 \mathrm{H}), 2.36-2.24(\mathrm{~m}, 1 \mathrm{H}), 2.11-1.96(\mathrm{~m}, 2 \mathrm{H}), 1.95-1.81$ $(\mathrm{m}, 4 \mathrm{H}), 1.80-1.68(\mathrm{~m}, 1 \mathrm{H})$ and $1.34-1.27(\mathrm{~m}, 3 \mathrm{H}) \mathrm{ppm}$.

${ }^{13} \mathrm{C}$ NMR $\left(100 \mathrm{MHz}, \mathrm{CDCl}_{3}\right)$ : Major isomer: $\delta_{\mathrm{C}} 165.4,137.05,136.36,130.06,128.72,127.2$, $106.6,81.0,75.3,68.3,60.5,56.6,35.3,27.01$ and $14.4 \mathrm{ppm}$.

Minor isomer: $\delta_{\mathrm{C}} 165.48,136.96,136.46,130.09,128.75,122.9,106.3,81.6,77.3,68.6,60.6$, 55.9, 36.5, 27.8 and $14.4 \mathrm{ppm}$.

IR (ATR): 3433, 2940, 1710, 1599, 1449, 1370, 1267, 1174, 1092, 1028, 970 and $770 \mathrm{~cm}^{-1}$.

HRMS (ESI) m/z: [M + H] ${ }^{+}$Calcd for $\mathrm{C}_{20} \mathrm{H}_{25} \mathrm{O}_{4}$ 329.1747; found 329.1691. 
TLC: $\mathrm{R}_{\mathrm{f}}=0.15(9: 1, \mathrm{Hex} / \mathrm{EtOAc})$.

ethyl (Z)-5-(1-hydroxy-4-methoxycyclohexyl)-3-(4-methoxyphenyl)pent-2-en-4-ynoate (9d) (Inseparable mixture of diastereomers with d.r. 1:0.8)

According to the General Procedure 8, (Z)-vinyliodide 23 (302 mg, 1 mmol, 1 equiv.), 1ethynyl-4-methoxycyclohexan-1-ol 26 (154 mg, 1 mmol, 1 equiv.), CuI (19 mg, 0.1 mmol, 10 $\mathrm{mol} \%), \mathrm{Pd}\left(\mathrm{PPh}_{3}\right)_{2} \mathrm{Cl}_{2}(9 \mathrm{mg}, 0.013 \mathrm{mmol}, 1.3 \mathrm{~mol} \%)$ and $0.5 \mathrm{~mL}$ of triethylamine were used in $15 \mathrm{~mL}$ THF, affording the desired propargylic alcohol 9d as a pale yellow oil (337 mg, 0.94 mmol, 94\%) by using Hexane/EtOAc (4:1) as eluent.

${ }^{1} \mathbf{H}$ NMR $\left(400 \mathrm{MHz}, \mathrm{CDCl}_{3}\right)$ : Major isomer: $\delta_{\mathrm{H}} 7.66(\mathrm{~d}, J=8.0$ $\mathrm{Hz}, 2 \mathrm{H}), 6.90$ (d, $J=8.0 \mathrm{~Hz}, 2 \mathrm{H}), 6.47(1 \mathrm{H}, \mathrm{s}), 4.24$ (q, $J=8.0$ $\mathrm{Hz}, 2 \mathrm{H},), 3.84(\mathrm{~s}, 3 \mathrm{H}), 3.31(\mathrm{~s}, 3 \mathrm{H}), 3.32-3.26(\mathrm{~m}, 1 \mathrm{H}), 2.25-$ $2.12(\mathrm{~m}, 1 \mathrm{H}), 2.10-1.97(\mathrm{~m}, 2 \mathrm{H}), 1.95-1.85(\mathrm{~m}, 3 \mathrm{H}), 1.80-$

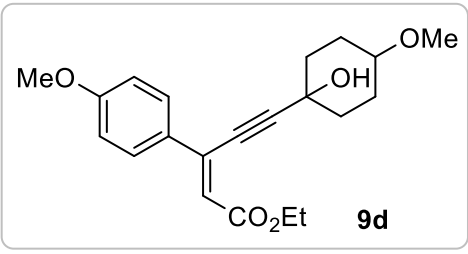
$1.68(\mathrm{~m}, 3 \mathrm{H})$ and $1.31(\mathrm{t}, J=8.0 \mathrm{~Hz}, 3 \mathrm{H}) \mathrm{ppm}$.

Minor isomer: $\delta_{\mathrm{H}} 7.66(\mathrm{~d}, J=8.0 \mathrm{~Hz}, 2 \mathrm{H}), 6.90(\mathrm{~d}, J=8.0 \mathrm{~Hz}, 2 \mathrm{H}), 6.47(1 \mathrm{H}, \mathrm{s}), 4.24(\mathrm{q}, J=$ $8.0 \mathrm{~Hz}, 2 \mathrm{H},), 3.84$ (s, 3H), $3.42-3.36(\mathrm{~m}, 1 \mathrm{H}), 3.33$ (s, 3H), $2.25-2.12(\mathrm{~m}, 1 \mathrm{H}), 2.10-1.97$ (m, 2H), $1.95-1.85(\mathrm{~m}, 3 \mathrm{H}), 1.80-1.68(\mathrm{~m}, 3 \mathrm{H})$ and $1.31(\mathrm{t}, J=8.0 \mathrm{~Hz}, 3 \mathrm{H}) \mathrm{ppm}$.

${ }^{13} \mathrm{C}$ NMR $\left(100 \mathrm{MHz}, \mathrm{CDCl}_{3}\right): \delta_{\mathrm{C}} 165.7,165.6,161.4,161.3,135.8,135.7,129.3,129.2,128.7$, 121.0, 114.2, 114.1, 105.9, 105.8, 82.0, 81.3, 75.3, 68.9, 68.5, 60.4, 60.4, 56.0, 55.7, 55.5, 36.6, $35.4,29.8,27.9,27.1$ and $14.5 \mathrm{ppm}$.

IR (ATR): 3436, 3056, 2936, 2862, 1711, 1601, 1511, 1454, 1262, 1171, 1092 and $739 \mathrm{~cm}^{-1}$.

HRMS (ESI) m/z: [M + H] $]^{+}$Calcd for $\mathrm{C}_{21} \mathrm{H}_{27} \mathrm{O}_{5}$ 359.1853; found 359.1850.

TLC: $\mathrm{R}_{\mathrm{f}}=0.15(9: 1, \mathrm{Hex} / \mathrm{EtOAc})$.

ethyl (Z)-5-(1-hydroxycycloheptyl)-3-phenylpent-2-en-4-ynoate (9e)

According to the General Procedure 8, (Z)-enol triflate ${ }^{3}$ (324 mg, $1 \mathrm{mmol}, 1$ equiv.), 1 ethynylcycloheptan-1-ol ${ }^{5}$ (138 mg, $1 \mathrm{mmol}, 1$ equiv.), CuI (19 mg, $\left.0.1 \mathrm{mmol}, 10 \mathrm{~mol} \%\right)$, $\mathrm{Pd}\left(\mathrm{PPh}_{3}\right)_{2} \mathrm{Cl}_{2}(9 \mathrm{mg}, 0.013 \mathrm{mmol}, 1.3 \mathrm{~mol} \%)$ and $0.5 \mathrm{~mL}$ of triethylamine were used in $15 \mathrm{~mL}$ THF, affording the desired propargylic alcohol 9e as a pale yellow oil (287 mg, $0.92 \mathrm{mmol}$, $92 \%)$ by using Hexane/EtOAc (4:1) as eluent. 
${ }^{1} \mathbf{H}$ NMR $\left(400 \mathrm{MHz}, \mathrm{CDCl}_{3}\right): \delta_{\mathrm{H}} 7.75-7.63(\mathrm{~m}, 2 \mathrm{H}), 7.43-7.31(\mathrm{~m}$, $3 \mathrm{H}), 6.52(\mathrm{~s}, 1 \mathrm{H}), 4.24$ (q, $J=8.0 \mathrm{~Hz}, 2 \mathrm{H}), 3.41$ (br s, 1H), $2.23-2.15$ $(\mathrm{m}, 2 \mathrm{H}), 2.03-1.94(\mathrm{~m}, 2 \mathrm{H}), 1.77-1.57(\mathrm{~m}, 8 \mathrm{H})$ and $1.30(\mathrm{t}, J=8.0$ $\mathrm{Hz}, 3 \mathrm{H}) \mathrm{ppm}$.

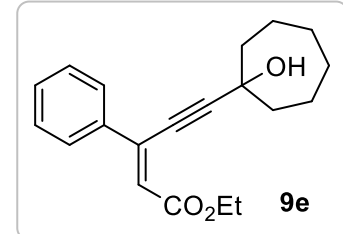

${ }^{13}$ C NMR $\left(100 \mathrm{MHz}, \mathrm{CDCl}_{3}\right): \delta_{\mathrm{C}} 165.5,137.2,136.5,130.0,128.7,127.2,122.7,108.1,80.7$, $72.3,60.5,42.8,28.3,22.2$ and $14.4 \mathrm{ppm}$.

IR (ATR): 3432, 2934, 2856, 2370, 2211, 1699, 1450, 1377, 1266, 1168, 1074, 1022, 961, 861 and $775 \mathrm{~cm}^{-1}$.

HRMS (ESI) m/z: [M + Na $]^{+}$Calcd for $\mathrm{C}_{20} \mathrm{H}_{24} \mathrm{NaO}_{3}$ 335.1618; found 335.1639.

TLC: $\mathrm{R}_{\mathrm{f}}=0.15(9: 1, \mathrm{Hex} / \mathrm{EtOAc})$.

ethyl (Z)-5-(1-hydroxycyclooctyl)-3-(4-methoxyphenyl)pent-2-en-4-ynoate (9f)

According to the General Procedure 8, (Z)-vinyliodide 23 (302 mg, 1 mmol, 1 equiv.), 1ethynylcyclooctan-1-ol ${ }^{6}$ (152 mg, 1 mmol, 1 equiv.), CuI (19 mg, $\left.0.1 \mathrm{mmol}, 10 \mathrm{~mol} \%\right)$, $\mathrm{Pd}\left(\mathrm{PPh}_{3}\right)_{2} \mathrm{Cl}_{2}(9 \mathrm{mg}, 0.013 \mathrm{mmol}, 1.3 \mathrm{~mol} \%)$ and $0.5 \mathrm{~mL}$ of triethylamine were used in $15 \mathrm{~mL}$ THF, affording the desired propargylic alcohol 9 f as a pale yellow oil (314 mg, $0.88 \mathrm{mmol}$, $88 \%)$ by using Hexane/EtOAc (4:1) as eluent.

${ }^{1}$ H NMR $\left(400 \mathrm{MHz}, \mathrm{CDCl}_{3}\right): \delta_{\mathrm{H}} 7.44(\mathrm{~d}, J=8.0 \mathrm{~Hz}, 2 \mathrm{H}), 6.86(\mathrm{~d}$, $J=8.0 \mathrm{~Hz}, 2 \mathrm{H}), 6.46(\mathrm{~s}, 1 \mathrm{H}), 4.10(\mathrm{q}, J=8.0 \mathrm{~Hz}, 2 \mathrm{H}), 3.82(\mathrm{~s}, 3 \mathrm{H})$, $2.05-1.89(\mathrm{~m}, 5 \mathrm{H}), 1.74-1.61(\mathrm{~m}, 6 \mathrm{H}), 1.57-1.43(\mathrm{~m}, 4 \mathrm{H})$ and $1.18(\mathrm{t}, J=8.0 \mathrm{~Hz}, 3 \mathrm{H}) \mathrm{ppm}$.

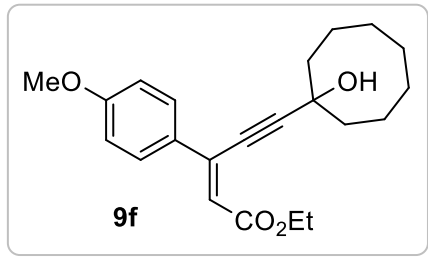

${ }^{13} \mathrm{C}$ NMR $\left(100 \mathrm{MHz}, \mathrm{CDCl}_{3}\right): \delta_{\mathrm{C}} 165.7,160.4,137.5,130.5,128.5,123.3,113.3,99.3,84.6$, $71.9,60.4,55.4,38.2,28.0,24.6,22.2$ and $14.2 \mathrm{ppm}$.

IR (ATR): 3436, 2926, 2857, 1712, 1598, 1511, 1457, 1364, 1259, 1171, 1032, 839 and 740 $\mathrm{cm}^{-1}$.

HRMS (ESI) m/z: [M + H] $]^{+}$Calcd for $\mathrm{C}_{22} \mathrm{H}_{29} \mathrm{O}_{4}$ 357.2060; found 357.2063.

TLC: $\mathrm{R}_{\mathrm{f}}=0.2(9: 1, \mathrm{Hex} / \mathrm{EtOAc})$.

ethyl (Z)-5-(1-hydroxycyclooctyl)-3-phenylpent-2-en-4-ynoate (9g)

According to the General Procedure 8, $(Z)$-enol triflate ${ }^{3}$ (324 mg, $1 \mathrm{mmol}, 1$ equiv.), 1 ethynylcyclooctan-1-ol ${ }^{6}$ (152 mg, 1 mmol, 1 equiv.), CuI (19 mg, $0.1 \mathrm{mmol}, 10 \mathrm{~mol} \%$ ), $\mathrm{Pd}\left(\mathrm{PPh}_{3}\right)_{2} \mathrm{Cl}_{2}(9 \mathrm{mg}, 0.013 \mathrm{mmol}, 1.3 \mathrm{~mol} \%)$ and $0.5 \mathrm{~mL}$ of triethylamine were used in $15 \mathrm{~mL}$ 
THF, affording the desired propargylic alcohol $9 \mathrm{~g}$ as a pale yellow oil (277 $\mathrm{mg}, 0.85 \mathrm{mmol}$, $85 \%)$ by using Hexane/EtOAc (4:1) as eluent.

${ }^{1}$ H NMR $\left(400 \mathrm{MHz}, \mathrm{CDCl}_{3}\right): \delta_{\mathrm{H}} 7.75-7.64(\mathrm{~m}, 2 \mathrm{H}), 7.44-7.31(\mathrm{~m}$, $3 \mathrm{H}), 6.54(\mathrm{~s}, 1 \mathrm{H}), 4.26(\mathrm{q}, J=8.0 \mathrm{~Hz}, 2 \mathrm{H}), 2.57$ (br s, 1H), $2.15-2.03$ $(\mathrm{m}, 4 \mathrm{H}), 1.79-1.59(\mathrm{~m}, 9 \mathrm{H}), 1.58-1.48(\mathrm{~m}, 3 \mathrm{H})$ and $1.33(3 \mathrm{H}, \mathrm{t}, J=$ $8.0 \mathrm{~Hz}) \mathrm{ppm}$.

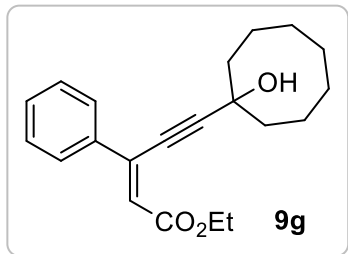

${ }^{13} \mathrm{C}$ NMR $\left(100 \mathrm{MHz}, \mathrm{CDCl}_{3}\right): \delta_{\mathrm{C}} 165.4,137.2,136.5,129.9,128.6,127.2,122.6,108.0,80.4$, $71.8,60.5,37.8,28.0,24.6,22.1$ and $14.4 \mathrm{ppm}$.

IR (ATR): 3436, 2925, 2860, 2361, 2211, 1696, 1450, 1380, 1269, 1180, 1074, 1022, 961, 844 and $775 \mathrm{~cm}^{-1}$.

HRMS (ESI) m/z: [M+ H] ${ }^{+}$Calcd for $\mathrm{C}_{21} \mathrm{H}_{27} \mathrm{O}_{3}$ 327.1955; found 327.1945.

TLC: $\mathrm{R}_{\mathrm{f}}=0.2(9: 1, \mathrm{Hex} / \mathrm{EtOAc})$.

ethyl (Z)-6-hydroxy-6-methyl-3-phenylhept-2-en-4-ynoate (9h)

According to the General Procedure 8, (Z)-enol triflate ${ }^{3}$ (324 mg, $1 \mathrm{mmol}, 1$ equiv.), 2methylbut-3-yn-2-ol (84 mg, $1 \mathrm{mmol}, 1$ equiv.), CuI (19 mg, $0.1 \mathrm{mmol}, 10 \mathrm{~mol} \%$ ), $\mathrm{Pd}\left(\mathrm{PPh}_{3}\right)_{2} \mathrm{Cl}_{2}(9 \mathrm{mg}, 0.013 \mathrm{mmol}, 1.3 \mathrm{~mol} \%)$ and $0.5 \mathrm{~mL}$ of triethylamine were used in $15 \mathrm{~mL}$ THF, affording the desired propargylic alcohol $\mathbf{9 h}$ as a yellow oil (219 $\mathrm{mg}, 0.85 \mathrm{mmol}, 85 \%)$ by using Hexane/EtOAc (4:1) as eluent.

${ }^{1} \mathbf{H}$ NMR $\left(400 \mathrm{MHz}, \mathrm{CDCl}_{3}\right): \delta_{\mathrm{H}} 7.72-7.62(\mathrm{~m}, 2 \mathrm{H}), 7.41-7.29(\mathrm{~m}, 3 \mathrm{H})$, $6.50(\mathrm{~s}, 1 \mathrm{H}), 4.39$ (br s, $1 \mathrm{H}), 4.22(\mathrm{q}, J=8.0 \mathrm{~Hz}, 2 \mathrm{H}), 1.67(\mathrm{~s}, 6 \mathrm{H})$ and $1.28(\mathrm{t}, J=8.0 \mathrm{~Hz}, 3 \mathrm{H}) \mathrm{ppm}$.

${ }^{13} \mathrm{C}$ NMR $\left(100 \mathrm{MHz}, \mathrm{CDCl}_{3}\right): \delta_{\mathrm{C}} 165.5,136.9,136.5,129.9,128.6,127.1$,

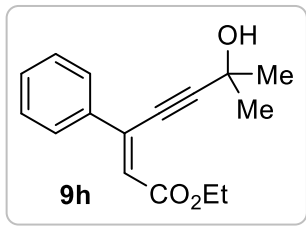
$122.4,107.8,79.2,65.4,60.5,31.0$ and $14.3 \mathrm{ppm}$.

IR (ATR): 3415, 2984, 1699, 1532, 1409, 1199, 1022, 954, 833 and $593 \mathrm{~cm}^{-1}$.

HRMS (ESI) m/z: [M + Na] ${ }^{+}$Calcd for $\mathrm{C}_{16} \mathrm{H}_{18} \mathrm{NaO}_{3} 281.1148$; found 281.1174.

TLC: $\mathrm{R}_{\mathrm{f}}=0.15(9: 1, \mathrm{Hex} / \mathrm{EtOAc})$.

ethyl (Z)-6-hydroxy-6,7-dimethyl-3-phenyloct-2-en-4-ynoate (9i)

According to the General Procedure 8, (Z)-enol triflate ${ }^{3}$ (324 mg, $1 \mathrm{mmol}, 1$ equiv.), 3,4dimethylpent-1-yn-3-ol (112 mg, $1 \mathrm{mmol}, 1$ equiv.), CuI (19 mg, $0.1 \mathrm{mmol}, 10 \mathrm{~mol} \%$ ), 
$\mathrm{Pd}\left(\mathrm{PPh}_{3}\right)_{2} \mathrm{Cl}_{2}(9 \mathrm{mg}, 0.013 \mathrm{mmol}, 1.3 \mathrm{~mol} \%)$ and $0.5 \mathrm{~mL}$ of triethylamine were used in $15 \mathrm{~mL}$ THF, affording the desired propargylic alcohol 9i as a yellow oil (254 $\mathrm{mg}, 0.89 \mathrm{mmol}, 89 \%)$ by using Hexane/EtOAc (5:1) as eluent.

${ }^{1} \mathbf{H}$ NMR $\left(400 \mathrm{MHz}, \mathrm{CDCl}_{3}\right): \delta_{\mathrm{H}} 7.75-7.63(\mathrm{~m}, 2 \mathrm{H}), 7.43-7.31(3 \mathrm{H}$, m), $6.53(\mathrm{~s}, 1 \mathrm{H}), 4.24$ (q, $J=8.0 \mathrm{~Hz}, 2 \mathrm{H}), 3.19$ (br s, 1H), $2.0-1.90$ (m, $1 \mathrm{H}), 1.58(\mathrm{~s}, 3 \mathrm{H}), 1.31(\mathrm{t}, J=7.1 \mathrm{~Hz}, 3 \mathrm{H}), 1.13(\mathrm{~d}, J=8.0 \mathrm{~Hz}, 3 \mathrm{H})$ and $1.09(\mathrm{~d}, J=8.0 \mathrm{~Hz}, 3 \mathrm{H}) \mathrm{ppm}$.

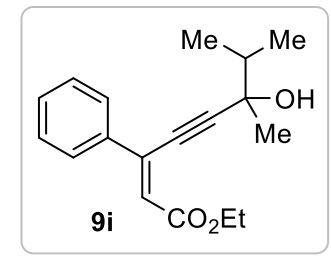

${ }^{13} \mathrm{C}$ NMR $\left(100 \mathrm{MHz}, \mathrm{CDCl}_{3}\right): \delta_{\mathrm{C}} 165.4,137.1,136.3,130.0,128.7,127.2,123.0,106.3,81.5$, $72.4,60.5,39.1,26.9,18.0,17.8$ and $14.4 \mathrm{ppm}$.

IR (ATR): 3412, 2978, 1762, 1701, 1606, 1400, 1218, 1170, 1022, 830 and $596 \mathrm{~cm}^{-1}$.

HRMS (ESI) m/z: [M + Na] $]^{+}$Calcd for $\mathrm{C}_{18} \mathrm{H}_{22} \mathrm{NaO}_{3}$ 309.1461; found 309.1470.

TLC: $\mathrm{R}_{\mathrm{f}}=0.2(9: 1, \mathrm{Hex} / \mathrm{EtOAc})$.

ethyl (Z)-6-hydroxy-6,8-dimethyl-3-phenylnon-2-en-4-ynoate (9j)

According to the General Procedure 8, (Z)-enol triflate ${ }^{3}$ (324 mg, $1 \mathrm{mmol}, 1$ equiv.), 3,5dimethylhex-1-yn-3-ol (126 mg, $1 \mathrm{mmol}, 1$ equiv.), CuI (19 mg, $0.1 \mathrm{mmol}, 10 \mathrm{~mol} \%$ ), $\mathrm{Pd}\left(\mathrm{PPh}_{3}\right)_{2} \mathrm{Cl}_{2}(9 \mathrm{mg}, 0.013 \mathrm{mmol}, 1.3 \mathrm{~mol} \%)$ and $0.5 \mathrm{~mL}$ of triethylamine were used in $15 \mathrm{~mL}$ THF, affording the desired propargylic alcohol $\mathbf{9 j}$ as a pale yellow oil (270 $\mathrm{mg}, 0.9 \mathrm{mmol}, 90 \%)$ by using Hexane/EtOAc $(5: 1)$ as eluent.

${ }^{1} \mathbf{H}$ NMR $\left(400 \mathrm{MHz}, \mathrm{CDCl}_{3}\right): \delta_{\mathrm{H}} 7.71-7.65(\mathrm{~m}, 2 \mathrm{H}), 7.38-7.32(\mathrm{~m}, 3 \mathrm{H})$, $6.50(\mathrm{~s}, 1 \mathrm{H}), 4.22(\mathrm{q}, J=8.0 \mathrm{~Hz}, 2 \mathrm{H}), 4.09(\mathrm{br} \mathrm{s}, 1 \mathrm{H}), 2.10-2.00(\mathrm{~m}, 1 \mathrm{H})$, $1.81-1.69(\mathrm{~m}, 2 \mathrm{H}), 1.65(\mathrm{~s}, 3 \mathrm{H}), 1.28(\mathrm{t}, J=8.0 \mathrm{~Hz}, 3 \mathrm{H})$ and $1.03(\mathrm{t}, J=$ $8.0 \mathrm{~Hz}, 6 \mathrm{H}) \mathrm{ppm}$.

${ }^{13}$ C NMR $\left(100 \mathrm{MHz}, \mathrm{CDCl}_{3}\right): \delta_{\mathrm{C}} 165.4,136.9,136.5,129.9,128.5,127.1$,

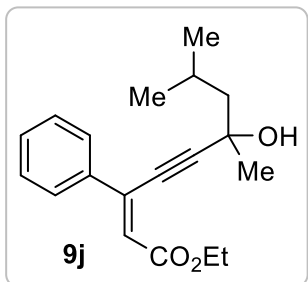
122.4, 107.7, 80.6, 68.4, 60.4, 51.6, 30.4, 25.1, 24.3, 24.2 and $14.3 \mathrm{ppm}$.

IR (ATR): 3425, 2978, 1700, 1529, 1401, 1205, 1015, 951, 833 and $599 \mathrm{~cm}^{-1}$.

HRMS (ESI) m/z: [M + Na] $]^{+}$Calcd for $\mathrm{C}_{19} \mathrm{H}_{24} \mathrm{NaO}_{3}$ 323.1618; found 323.1628.

TLC: $\mathrm{R}_{\mathrm{f}}=0.3(9: 1, \mathrm{Hex} / \mathrm{EtOAc})$. 


\section{ethyl (Z)-6-ethyl-6-hydroxy-3-phenyldec-2-en-4-ynoate (9k)}

According to the General Procedure 8, $(Z)$-enol triflate ${ }^{3}$ (324 mg, 1 mmol, 1 equiv.), 3ethylhept-1-yn-3-ol (140 mg, $1 \mathrm{mmol}, 1$ equiv.), CuI (19 mg, $0.1 \mathrm{mmol}, 10 \mathrm{~mol} \%)$, $\mathrm{Pd}\left(\mathrm{PPh}_{3}\right)_{2} \mathrm{Cl}_{2}(9 \mathrm{mg}, 0.013 \mathrm{mmol}, 1.3 \mathrm{~mol} \%)$ and $0.5 \mathrm{~mL}$ of triethylamine were used in $15 \mathrm{~mL}$ THF, affording the desired propargylic alcohol 9k as a pale yellow oil (267 $\mathrm{mg}, 0.85 \mathrm{mmol}$, $85 \%)$ by using Hexane/EtOAc $(5: 1)$ as eluent.

${ }^{1}$ H NMR $\left(400 \mathrm{MHz}, \mathrm{CDCl}_{3}\right): \delta_{\mathrm{H}} 7.74-7.64(\mathrm{~m}, 2 \mathrm{H}), 7.43-7.34(\mathrm{~m}$, $3 \mathrm{H}), 6.53$ (s, 1H), 4.24 (q, $J=8.0 \mathrm{~Hz}, 2 \mathrm{H}), 3.09$ (br s, 1H), $1.87-1.72$ (m, 4H), $1.63-1.53(\mathrm{~m}, 2 \mathrm{H}), 1.39$ (dt, $J=14.6,7.3 \mathrm{~Hz}, 2 \mathrm{H}), 1.31$ (t, $J$ $=7.1 \mathrm{~Hz}, 3 \mathrm{H}), 1.12(\mathrm{t}, J=7.4 \mathrm{~Hz}, 3 \mathrm{H})$ and $0.93(\mathrm{t}, J=7.3 \mathrm{~Hz}, 3 \mathrm{H}) \mathrm{ppm}$.

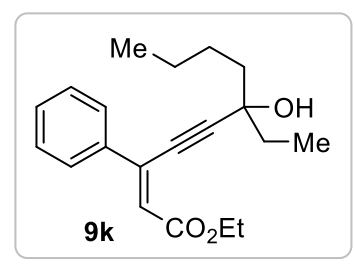

${ }^{13} \mathrm{C}$ NMR (100 MHz, $\left.\mathrm{CDCl}_{3}\right): \delta_{\mathrm{C}} 165.4,137.1,136.3,130.0,128.7,127.2,122.9,106.2,81.8$, $72.4,60.5,41.1,34.7,26.6,23.1,14.4,14.2$ and 8.8 ppm.

IR (ATR): 2960, 2938, 1706, 1599, 1372, 1266, 1177, 766, 751 and $692 \mathrm{~cm}^{-1}$.

HRMS (ESI) m/z: $[\mathrm{M}+\mathrm{H}]^{+}$Calcd for $\mathrm{C}_{20} \mathrm{H}_{27} \mathrm{O}_{3}$ 315.1955; found 315.1951.

TLC: $\mathrm{R}_{\mathrm{f}}=0.3$ (9:1, Hex/EtOAc).

\section{ethyl (Z)-5-(1-hydroxycyclohexyl)-3-(3-nitrophenyl)pent-2-en-4-ynoate (9l)}

According to the General Procedure 8, (Z)-vinyliodide 28 (347 mg, 1 mmol, 1 equiv.), 1ethynylcyclohexan-1-ol (124 mg, $1 \mathrm{mmol}, 1$ equiv.), CuI (19 mg, $0.1 \mathrm{mmol}, 0.1$ equiv.), $\mathrm{Pd}\left(\mathrm{PPh}_{3}\right)_{2} \mathrm{Cl}_{2}(9 \mathrm{mg}, 0.013 \mathrm{mmol}, 1.3 \mathrm{~mol} \%)$ and $0.5 \mathrm{~mL}$ of triethylamine were used in $15 \mathrm{~mL}$ THF, affording the desired propargylic alcohol 91 as a brown crystalline solid (305 $\mathrm{mg}, 0.89$ mmol, $89 \%$ ) by using Hexane/EtOAc (4:1) as eluent.

${ }^{1} \mathbf{H}$ NMR $\left(400 \mathrm{MHz}, \mathrm{CDCl}_{3}\right): \delta_{\mathrm{H}} 8.58(\mathrm{~s}, 1 \mathrm{H}), 8.25(\mathrm{~d}, J=8.1 \mathrm{~Hz}, 1 \mathrm{H})$, $8.03(\mathrm{~d}, J=7.8 \mathrm{~Hz}, 1 \mathrm{H}), 7.59(\mathrm{t}, J=8.0 \mathrm{~Hz}, 1 \mathrm{H}), 6.63(\mathrm{~s}, 1 \mathrm{H}), 4.28(\mathrm{q}, J$ $=7.0 \mathrm{~Hz}, 2 \mathrm{H}), 3.39(\mathrm{br} \mathrm{s}, 1 \mathrm{H}), 2.13-2.06(\mathrm{~m}, 2 \mathrm{H}), 1.78-1.51(\mathrm{~m}, 8 \mathrm{H})$ and $1.34(\mathrm{t}, J=7.0 \mathrm{~Hz}, 3 \mathrm{H}) \mathrm{ppm}$.

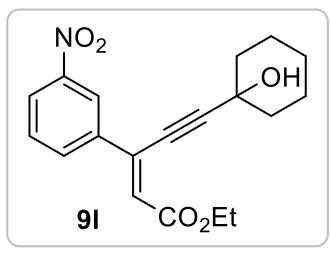

${ }^{13} \mathrm{C}$ NMR $\left(100 \mathrm{MHz}, \mathrm{CDCl}_{3}\right): \delta_{\mathrm{C}} 164.8,148.6,138.8,133.8,132.8,129.8,124.7,124.5,122.2$, $108.2,80.5,69.4,61.0,39.7,25.3,23.4$ and $14.4 \mathrm{ppm}$.

IR (ATR): 3055, 2987, 2939, 2305, 1717, 1534, 1444, 1422, 1350, 1265, 1184, 1167, 896, 739 and $706 \mathrm{~cm}^{-1}$.

HRMS (ESI) m/z: [M + H $]^{+}$Calcd for $\mathrm{C}_{19} \mathrm{H}_{22} \mathrm{NO}_{5} 344.1492$; found 344.1460. 
TLC: $\mathrm{R}_{\mathrm{f}}=0.25$ (4:1, Hex/EtOAc).

MP: $75-77{ }^{\circ} \mathrm{C}$ (recrystallized from $\mathrm{CH}_{2} \mathrm{Cl}_{2} /$ Hexane: $1: 1$ ).

\section{ethyl (Z)-5-(1-hydroxycyclohexyl)-3-(3-methoxyphenyl)pent-2-en-4-ynoate (9m)}

According to the General Procedure 8, (Z)-enol triflate ${ }^{7}$ (354 mg, $1 \mathrm{mmol}, 1$ equiv.), 1ethynylcyclohexan-1-ol (124 mg, $1 \mathrm{mmol}, 1$ equiv.), CuI (19 mg, $0.1 \mathrm{mmol}, 0.1$ equiv.), $\mathrm{Pd}\left(\mathrm{PPh}_{3}\right)_{2} \mathrm{Cl}_{2}$ (14 mg, $0.02 \mathrm{mmol}, 0.02$ equiv.) and $0.5 \mathrm{~mL}$ of triethylamine were used in 15 $\mathrm{mL}$ THF, affording the desired propargylic alcohol $\mathbf{9 m}$ as a pale yellow oil $(285 \mathrm{mg}, 0.87$ mmol, $87 \%$ ) by using Hexane/EtOAc (9:1) as eluent.

${ }^{1} \mathbf{H}$ NMR $\left(400 \mathrm{MHz}, \mathrm{CDCl}_{3}\right): \delta_{\mathrm{H}} 7.31-7.22(\mathrm{~m}, 3 \mathrm{H}), 6.97-6.89(\mathrm{~m}$, $1 \mathrm{H}), 6.51(1 \mathrm{H}, \mathrm{s}), 4.23(\mathrm{q}, J=8.0 \mathrm{~Hz}, 2 \mathrm{H}), 3.81(\mathrm{~s}, 3 \mathrm{H}), 3.87-3.64$ (br s, $1 \mathrm{H}), 2.13-2.04(\mathrm{~m}, 2 \mathrm{H}), 1.79-1.54(\mathrm{~m}, 7 \mathrm{H})$ and $1.30(\mathrm{t}, J=8.0 \mathrm{~Hz}, 3 \mathrm{H})$ ppm.

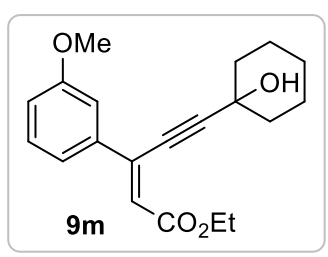

${ }^{13} \mathrm{C}$ NMR $\left(100 \mathrm{MHz}, \mathrm{CDCl}_{3}\right): \delta_{\mathrm{C}} 165.4,159.7,138.6,136.3,129.6,122.8,119.5,115.9,112.7$, $107.0,81.3,69.2,60.5,55.3,39.7,25.3,23.4$ and $14.4 \mathrm{ppm}$.

IR (ATR): 3440, 2939, 1712, 1593, 1454, 1281, 1219, 1169, 1041, 968, 864 and $787 \mathrm{~cm}^{-1}$.

HRMS (ESI) m/z: [M+ H] ${ }^{+}$Calcd for $\mathrm{C}_{20} \mathrm{H}_{25} \mathrm{O}_{4}$ 329.1747; found 329.1738.

TLC: $\mathrm{R}_{\mathrm{f}}=0.3$ (9:1, Hex/EtOAc).

10. Procedure 9: Procedure for preparation of propargyl alcohol (26):

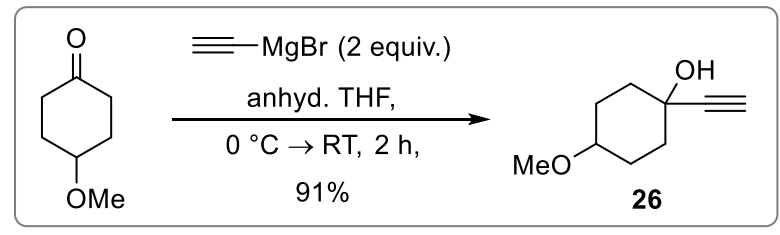

To the stirred solution of 4-methylcyclohexan-1-one ${ }^{8}$ (256 mg, $2 \mathrm{mmol}$, 1.0 equiv.) in anhydrous THF was added ethynyl magnesium bromide ( $2 \mathrm{~mL}, 2.0$ equiv., $0.5 \mathrm{M}$ in THF) drop wise at $0{ }^{\circ} \mathrm{C}$ under nitrogen. The mixture was stirred at same temperature for $2 \mathrm{~h}$. The reaction mixture was quenched with saturated $\mathrm{NH}_{4} \mathrm{Cl}$ solution. The aqueous layer was extracted with EtOAc (3 times). The combined organic layers were washed with brine, dried over anhydrous $\mathrm{Na}_{2} \mathrm{SO}_{4}$, filtered and concentrated in-vacuo. Purification of crude product via silica gel column chromatography (Hexanes/EA) provided a diastereomeric mixture (anti:syn $=3: 1)$ of the desired propargyl alcohol 26 as a colourless oil (280 mg, $1.8 \mathrm{mmol}, 91 \%)$. 
${ }^{1} \mathbf{H}$ NMR $\left(400 \mathrm{MHz}, \mathrm{CDCl}_{3}\right): \delta_{\mathrm{H}} 3.48-3.13(\mathrm{~m}, 4 \mathrm{H}), 2.86-2.36(\mathrm{~m}, 2 \mathrm{H})$, $2.22-1.69(\mathrm{~m}, 7 \mathrm{H})$ and $1.69-1.54(\mathrm{~m}, 1 \mathrm{H}) \mathrm{ppm}$.

${ }^{13} \mathrm{C}$ NMR $\left(100 \mathrm{MHz}, \mathrm{CDCl}_{3}\right): \delta_{\mathrm{C}} 87.6,87.2,75.2,72.7,71.7,68.0,67.5$,

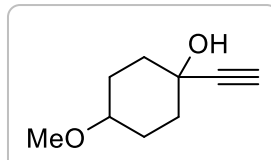

26 $55.9,55.6,36.7,35.4,27.7$ and $26.9 \mathrm{ppm}$.

HRMS (ESI) m/z: [M + Na] ${ }^{+}$Calcd for $\mathrm{C}_{9} \mathrm{H}_{14} \mathrm{NaO}_{2}$ 177.0886; found 177.0879.

TLC: $\mathrm{R}_{\mathrm{f}}=0.35$ (2:1, Hex/EtOAc).

\section{General procedure 10: for preparation of vinyl iodide:}

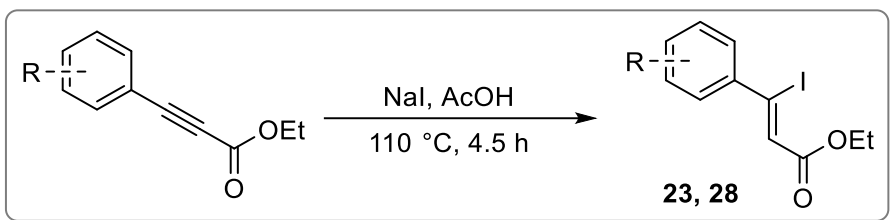

A mixture of propiolate ester derivative and sodium iodide in acetic acid was heated in a preheated oil bath at $110{ }^{\circ} \mathrm{C}$ for $4.5 \mathrm{~h}$. The mixture was cooled and partitioned between ethyl acetate and water. The layers were separated, and the aqueous layer was extracted with ethyl acetate. The combined extracts were washed with saturated aqueous sodium hydrogen carbonate, sodium thiosulfate, and brine, dried over $\mathrm{Na}_{2} \mathrm{SO}_{4}$, and filtered. Purification of crude product via silica gel column chromatography (Hexanes/EA) provided the desired vinyl iodide.

\section{ethyl (Z)-3-iodo-3-(4-methoxyphenyl)acrylate (23)}

According to the General Procedure 10, ethyl 3-(4-methoxyphenyl)propiolate ${ }^{9}$ (510 mg, 2.5 mmol, 1.0 equiv.) and sodium iodide (1.12 g, $7.5 \mathrm{mmol}, 3.0$ equiv.) were used in $1.9 \mathrm{~mL}$ (13 equiv.) acetic acid, affording the desired vinyl iodide $\mathbf{2 3}$ as a colourless oil (797 mg, $2.4 \mathrm{mmol}$, 96\%) by using Hexane/EtOAc (9:1) as eluent.

${ }^{1} \mathbf{H}$ NMR $\left(400 \mathrm{MHz}, \mathrm{CDCl}_{3}\right): \delta_{\mathrm{H}} 7.30(\mathrm{~d}, J=8.3 \mathrm{~Hz}, 2 \mathrm{H}), 6.81(\mathrm{~d}, J=$ $8.4 \mathrm{~Hz}, 2 \mathrm{H}), 6.57(\mathrm{~s}, 1 \mathrm{H}), 4.02(\mathrm{q}, J=7.0 \mathrm{~Hz}, 2 \mathrm{H}), 3.81(\mathrm{~s}, 3 \mathrm{H})$ and 1.12 (t, $J=7.1 \mathrm{~Hz}, 3 \mathrm{H}) \mathrm{ppm}$.

${ }^{13} \mathrm{C}$ NMR $\left(100 \mathrm{MHz}, \mathrm{CDCl}_{3}\right): \delta_{\mathrm{C}} 163.8,160.3,131.6,129.8,125.0$,

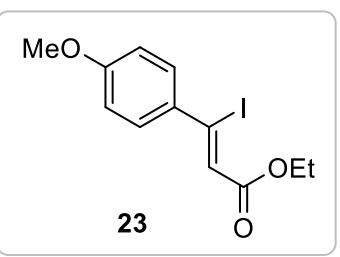
$113.2,111.5,80.3,60.6,55.4$ and $14.0 \mathrm{ppm}$.

HRMS (ESI) m/z: [M + H] $]^{+}$Calcd for $\mathrm{C}_{12} \mathrm{H}_{14} \mathrm{IO}_{3}$ 332.9982; found 332.9982 . 
TLC: $\mathrm{R}_{\mathrm{f}}=0.45$ (19:1, Hex/EtOAc).

ethyl (Z)-3-iodo-3-(3-nitrophenyl)acrylate (28)

According to the General Procedure 10, ethyl 3-(3-nitrophenyl)propiolate ${ }^{10}$ (548 mg, 2.5 mmol, 1.0 equiv.) and sodium iodide ( $1.2 \mathrm{~g}, 8.0 \mathrm{mmol}, 3.2$ equiv.) were used in $1.8 \mathrm{~mL}$ (12.8 equiv.) acetic acid, affording the desired vinyl iodide $\mathbf{2 8}$ as a yellow crystalline solid (824 mg, $2.4 \mathrm{mmol}, 95 \%)$ by using Hexane/EtOAc (9:1) as eluent.

${ }^{1} \mathbf{H}$ NMR $\left(400 \mathrm{MHz}, \mathrm{CDCl}_{3}\right): \delta_{\mathrm{H}} 8.38(\mathrm{~s}, 1 \mathrm{H}), 8.22(\mathrm{~d}, J=8.1 \mathrm{~Hz}, 1 \mathrm{H})$, $7.86(\mathrm{~d}, J=7.8 \mathrm{~Hz}, 1 \mathrm{H}), 7.58(\mathrm{t}, J=8.0 \mathrm{~Hz}, 1 \mathrm{H}), 6.73(\mathrm{~s}, 1 \mathrm{H}), 4.32(\mathrm{q}$, $J=7.1 \mathrm{~Hz}, 2 \mathrm{H})$ and $1.36(\mathrm{t}, J=7.1 \mathrm{~Hz}, 3 \mathrm{H}) \mathrm{ppm}$.

${ }^{13}$ C NMR (100 MHz, $\left.\mathrm{CDCl}_{3}\right): \delta_{\mathrm{C}} 164.2,148.1,145.0,134.6,129.7$,

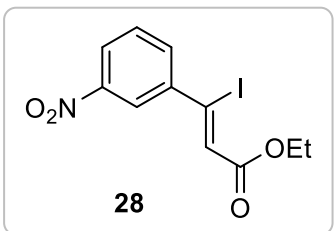
$129.3,124.5,123.5,111.6,61.3$ and $14.3 \mathrm{ppm}$.

IR (ATR): 3055, 2986, 1723, 1607, 1533, 1356, 1310, 1302, 1266, 1180, 1029, 897, 806, 738, $705 \mathrm{~cm}^{-1}$.

HRMS (ESI) m/z: $[\mathrm{M}+\mathrm{H}]^{+}$Calcd for $\mathrm{C}_{11} \mathrm{H}_{11} \mathrm{INO}_{4}$ 347.9727; found 347.9707 .

TLC: $\mathrm{R}_{\mathrm{f}}=0.75(4: 1, \mathrm{Hex} / \mathrm{EtOAc})$.

MP: $69-71{ }^{\circ} \mathrm{C}$ (recrystallized from hot hexanes). 


\section{References}

1. (a) Han, T.; Jiang, Y.; Ji, X.; Deng, G. J.; Huang, H. Aerobic C ( $\left.\mathrm{sp}^{3}\right)-\mathrm{H}$ oxidation and oxygenation of quaternarized quinolines and pyridines by visible-light-induced photocatalysis. Org. Chem. Front. 2020, 7, 1671-1678. (b) Uygur, M.; Kuhlmann, J. H.; Pérez-Aguilar, M. C.; Piekarski, D. G.; Mancheño, O. G. Metal-and additive-free C-H oxygenation of alkylarenes by visible-light photoredox catalysis. Green Chem. 2021, 23, 3392-3399.

2. (a) Miao, S.; Andersen, R. J. Rubrolides A-H, metabolites of the colonial tunicate Ritterella rubra. J. Org. Chem. 1991, 56, 6275-6280. (b) Karak, M.; Barbosa, L. C.; Maltha, C. R.; Silva, T. M.; Boukouvalas, J. Palladium-catalyzed hydrodehalogenation of butenolides: An efficient and sustainable access to $\beta$-arylbutenolides. Tetrahedron Lett. 2017, 58, 28302834.

3. Babinski, D.; Soltani, D.; Frantz, D. E. Stereoselective synthesis of acetoacetate-derived enol triflates. Org. Lett. 2008, 10, 2901-2904.

4. Sum, Y. N.; Yu, D.; Zhang, Y. Synthesis of acetylenic alcohols with calcium carbide as the acetylene source. Green Chem., 2013, 15, 2718-2721

5. Friese, F. W.; Studer, A. Deoxygenative Borylation of Secondary and Tertiary Alcohols. Angew. Chem., Int. Ed. 2019, 58, 9561-9564.

6. Kuang, Z.; Chen, H.; Yan, J.; Yang, K.; Lan, Y.; Song, Q. Base-catalyzed borylation/B-O elimination of propynols and $\mathrm{B}_{2} \mathrm{pin}_{2}$ delivering tetrasubstituted alkenylboronates. Org. Lett. 2018, 20, 5153-5157.

7. Maekawa, H.; Noda, K.; Kuramochi, K.; Zhang, T. Catalyst-free and solvent-controlled reductive coupling of activated vinyl triflates with chlorotrimethylsilane by magnesium metal and its synthetic application. Org. Lett. 2018, 20, 1953-1956.

8. Kayser, M. M.; Clouthier, C. M. new bioorganic reagents: evolved cyclohexanone monooxygenase why is it more selective? J. Org. Chem. 2006, 71, 8424-8430.

9. Cai, S.; Yang, K.; Wang, D. Z. Gold catalysis coupled with visible light stimulation: syntheses of functionalized indoles. Org. Lett. 2014, 16, 2606-2609.

10. Tarigopula, C.; Thota, G. K.; Balamurugan, R. Efficient synthesis of functionalized $\beta$-keto esters and $\beta$-diketones through regioselective hydration of alkynyl esters and alkynyl ketones by use of a cationic NHC-AuI catalyst. Eur. J. Org. Chem., 2016, 35, 5855-5861. 
12. Copy of ${ }^{1} \mathrm{H}$ and ${ }^{13} \mathrm{C}$ NMR spectra of all new compounds 
${ }^{1} \mathrm{H}$ NMR $\left(400 \mathrm{MHz}, \mathrm{CDCl}_{3}\right)$ and ${ }^{13} \mathrm{C} \mathrm{NMR}\left(100 \mathrm{MHz}, \mathrm{CDCl}_{3}\right)$ of 12a.
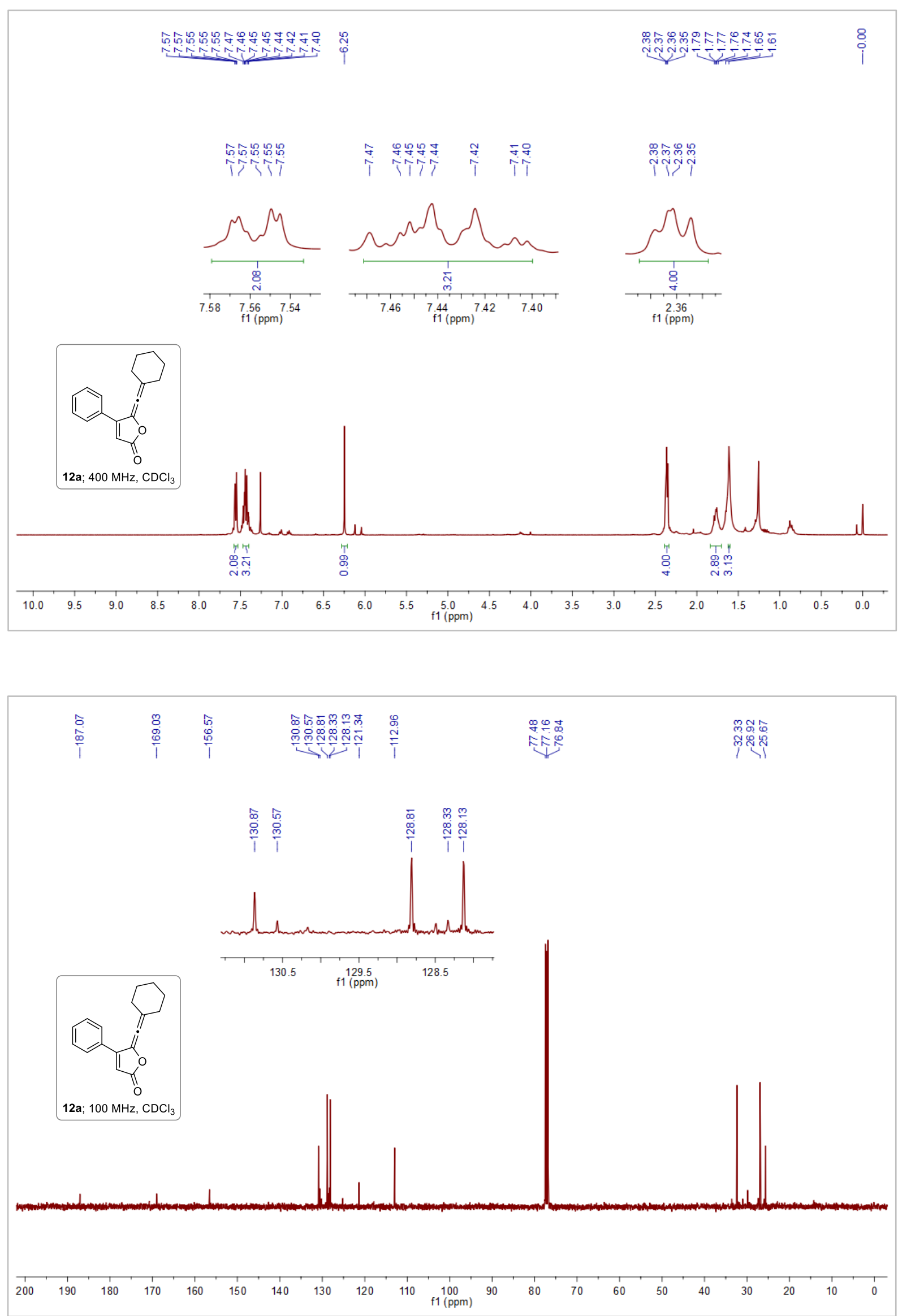
${ }^{1} \mathrm{H}$ NMR (400 MHz, $\left.\mathrm{CDCl}_{3}\right)$ and ${ }^{13} \mathrm{C}$ NMR $\left(100 \mathrm{MHz}, \mathrm{CDCl}_{3}\right)$ of $\mathbf{1 2 b}$.
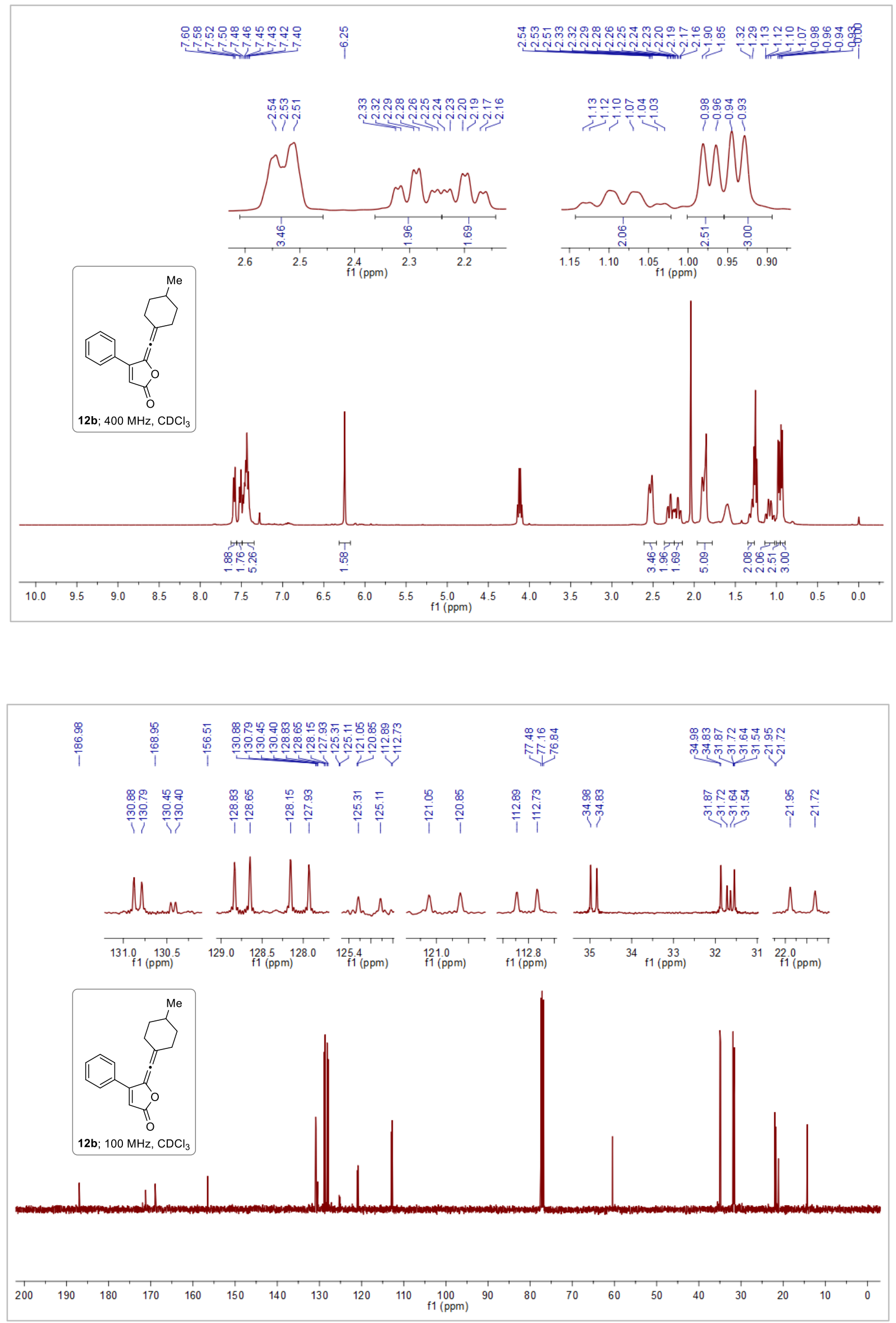
${ }^{1} \mathrm{H}$ NMR $\left(400 \mathrm{MHz}, \mathrm{CDCl}_{3}\right)$ and ${ }^{13} \mathrm{C}$ NMR $\left(100 \mathrm{MHz}, \mathrm{CDCl}_{3}\right)$ of $\mathbf{1 2 c}$.
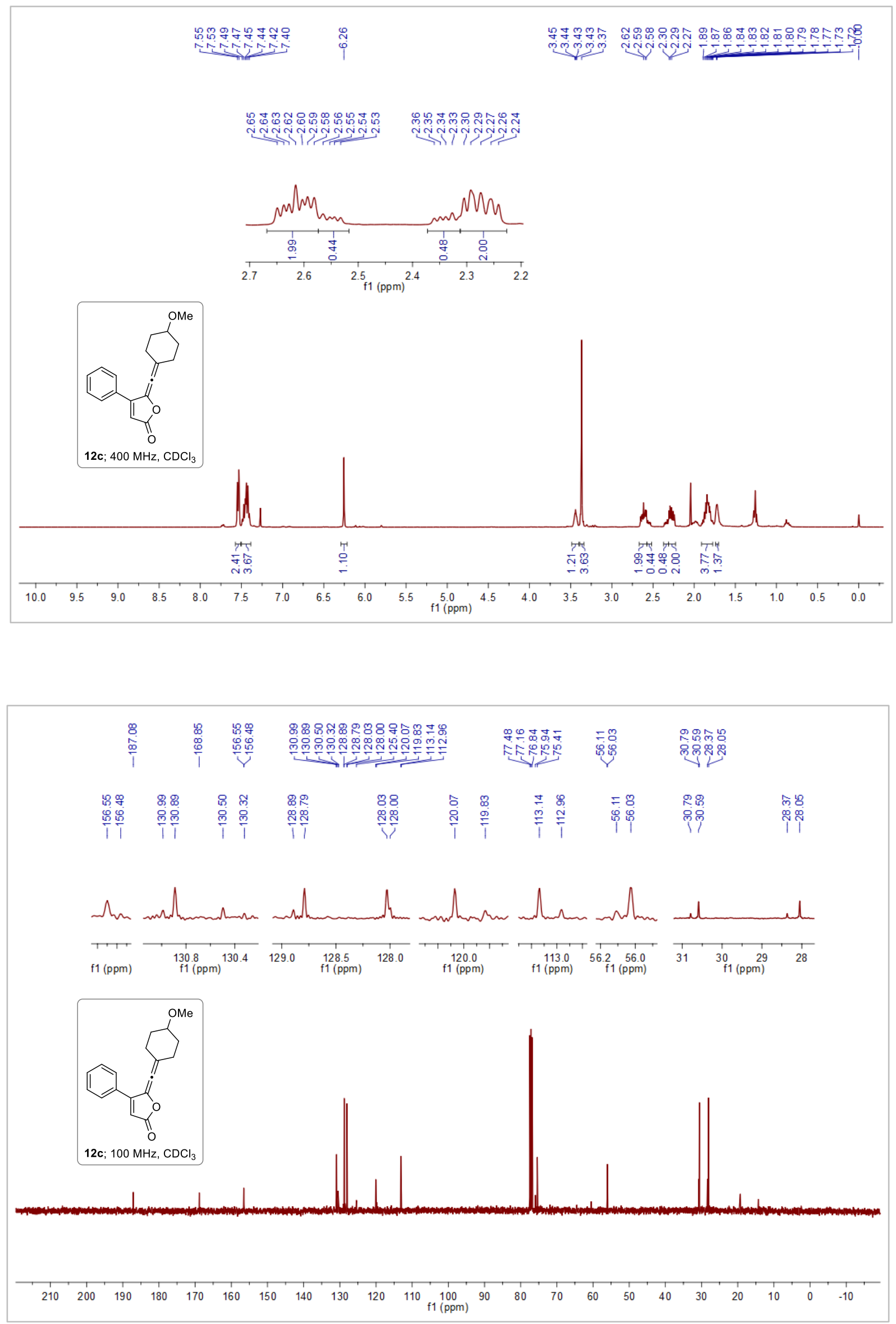
${ }^{1} \mathrm{H}$ NMR $\left(400 \mathrm{MHz}, \mathrm{CDCl}_{3}\right)$ and ${ }^{13} \mathrm{C}$ NMR $\left(100 \mathrm{MHz}, \mathrm{CDCl}_{3}\right)$ of $\mathbf{1 2 d}$.
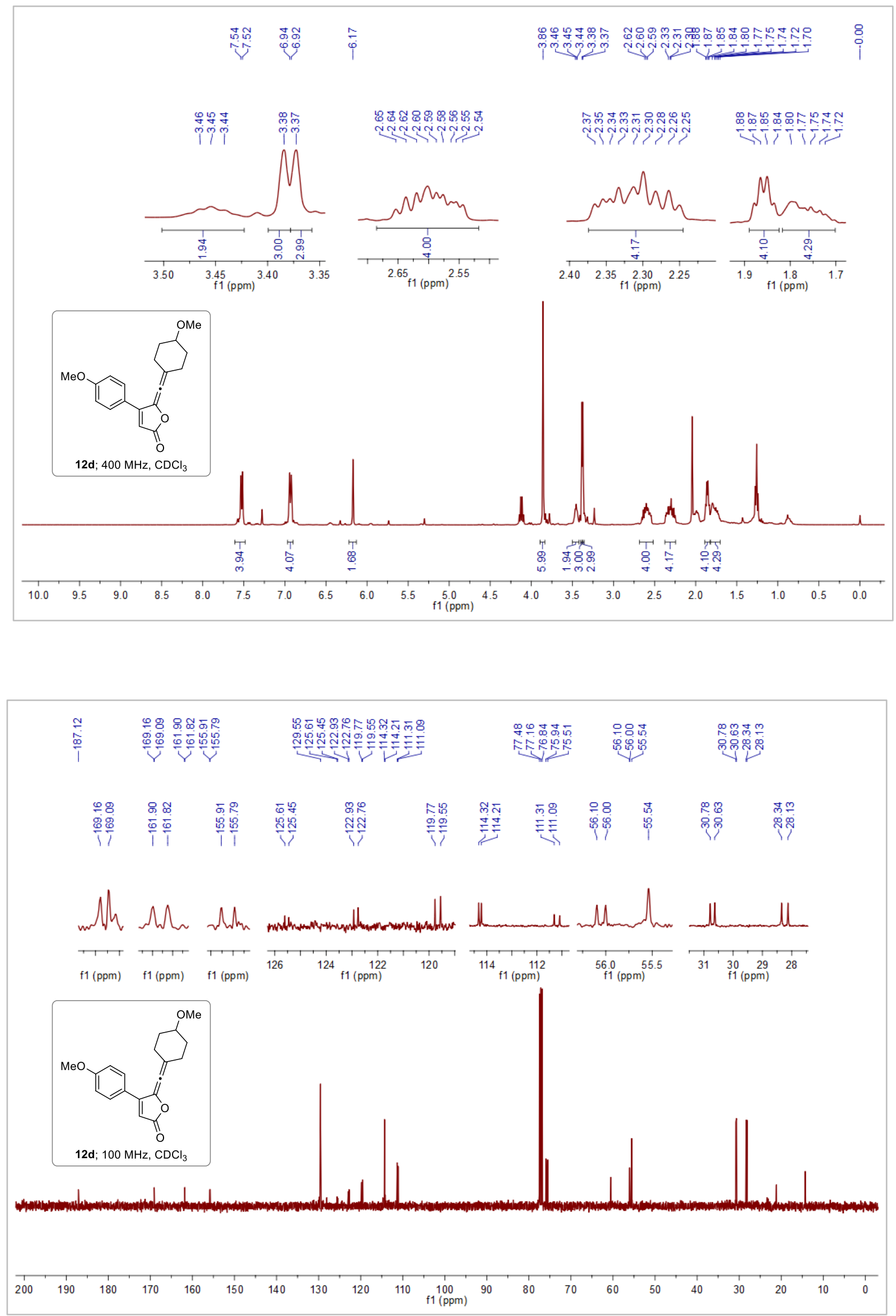
${ }^{1} \mathrm{H}$ NMR $\left(400 \mathrm{MHz}, \mathrm{CDCl}_{3}\right)$ and ${ }^{13} \mathrm{C}$ NMR $\left(100 \mathrm{MHz}, \mathrm{CDCl}_{3}\right)$ of $\mathbf{1 2 e}$.
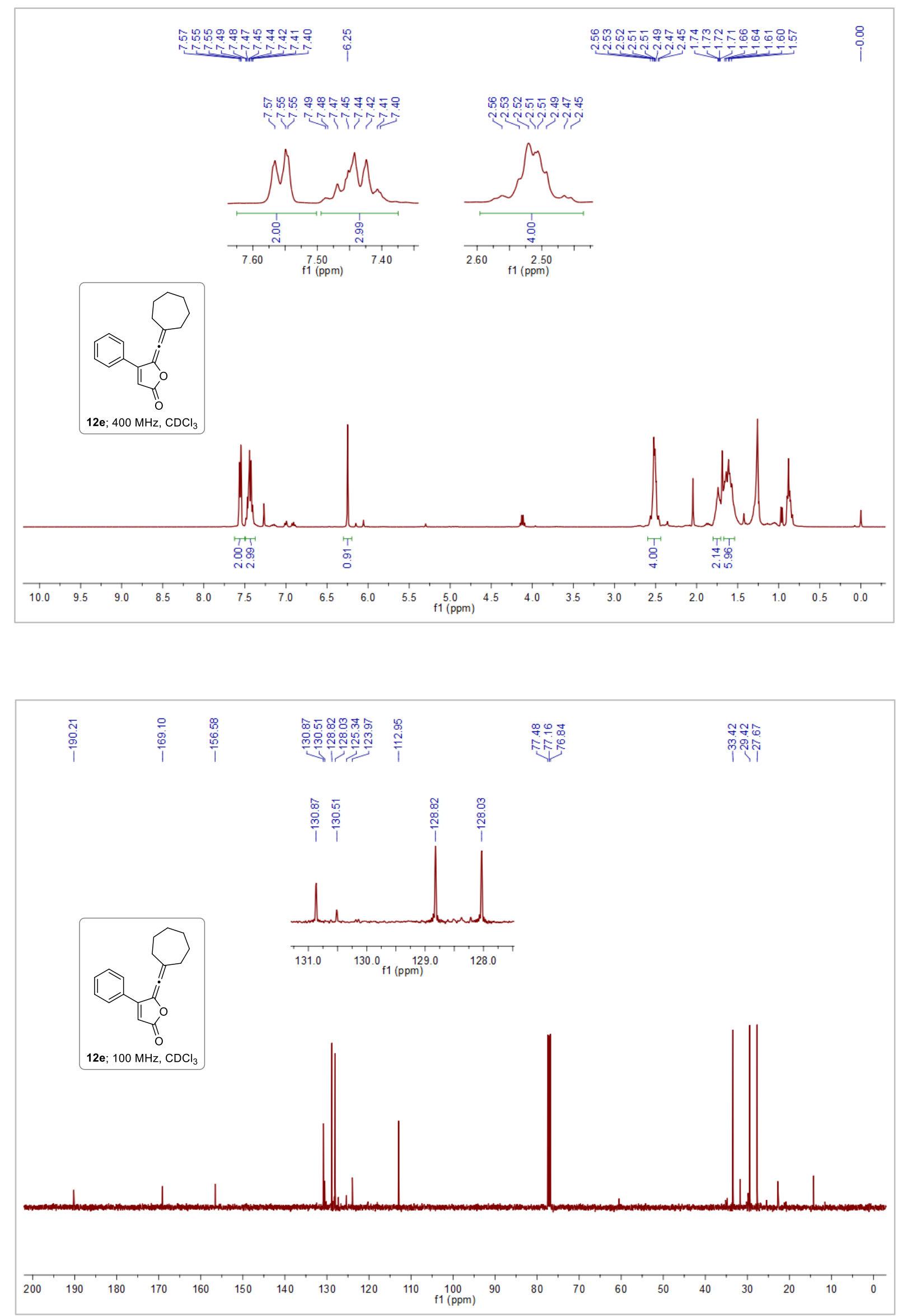
${ }^{1} \mathrm{H}$ NMR (400 MHz, $\left.\mathrm{CDCl}_{3}\right)$ and ${ }^{13} \mathrm{C}$ NMR $\left(100 \mathrm{MHz}, \mathrm{CDCl}_{3}\right)$ of $\mathbf{1 2 f}$.
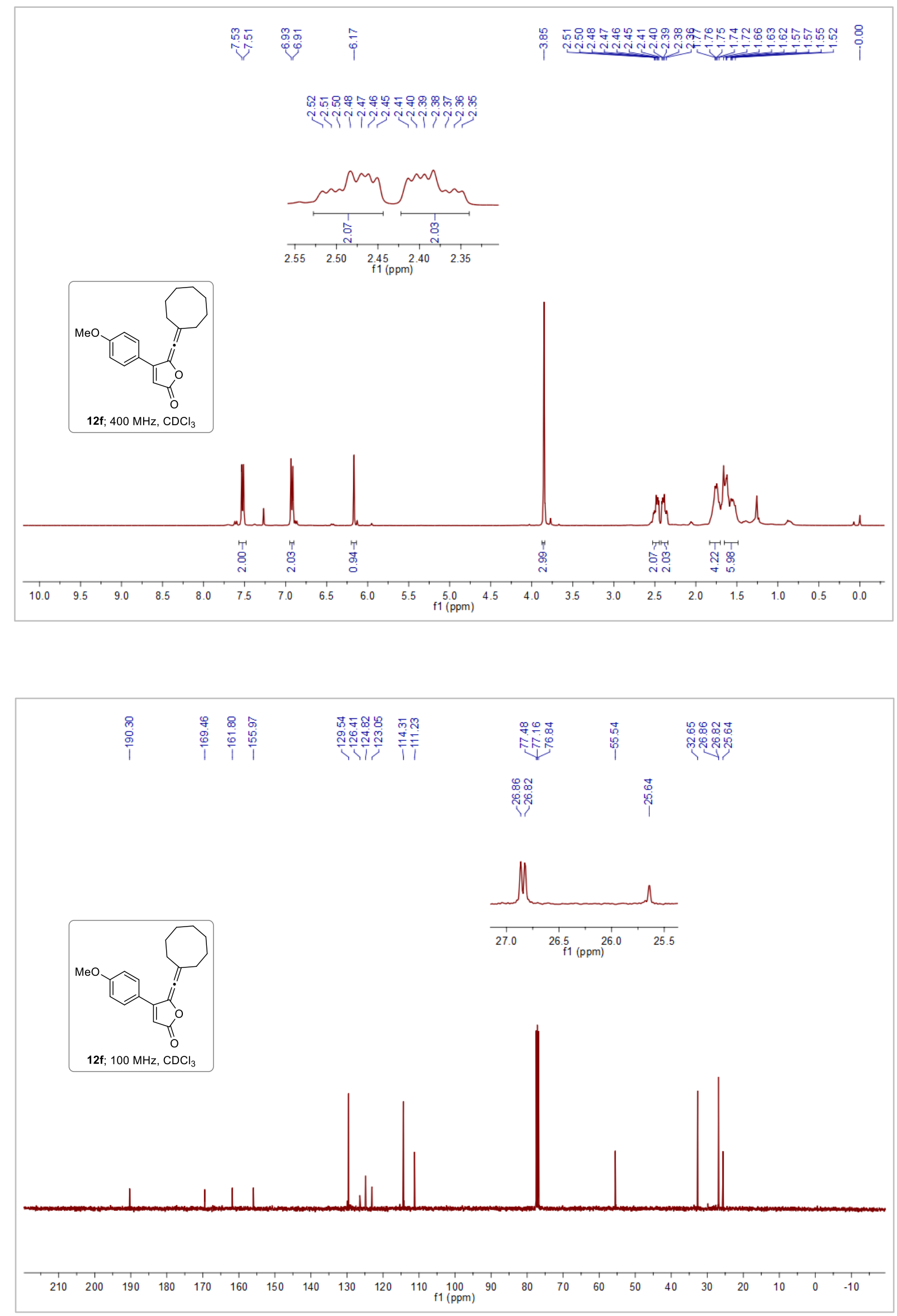
${ }^{1} \mathrm{H}$ NMR (400 MHz, $\left.\mathrm{CDCl}_{3}\right)$ and ${ }^{13} \mathrm{C}$ NMR (100 MHz, $\left.\mathrm{CDCl}_{3}\right)$ of 12g.
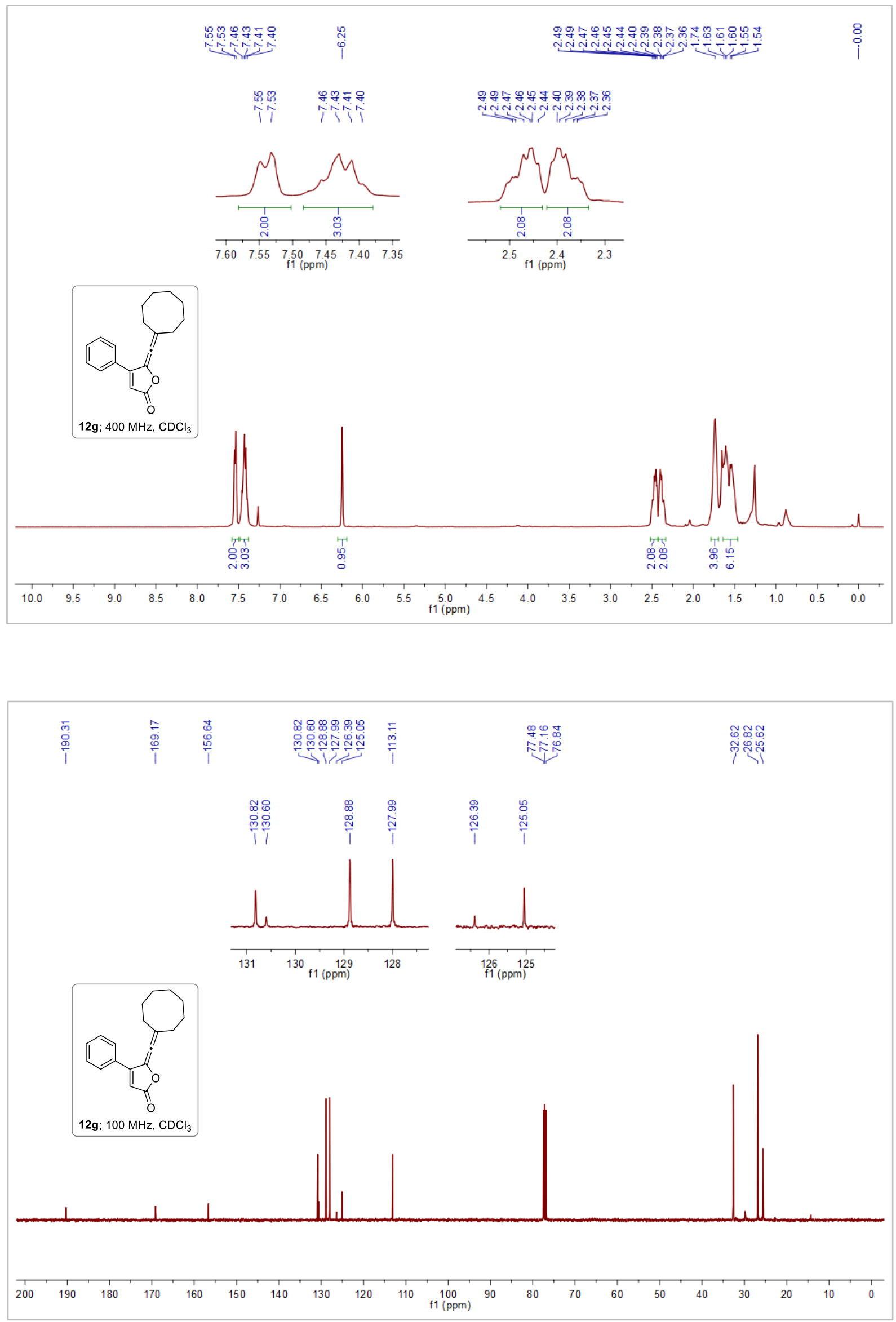


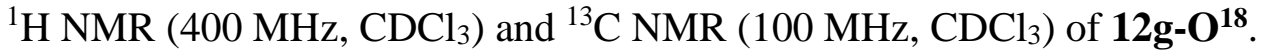
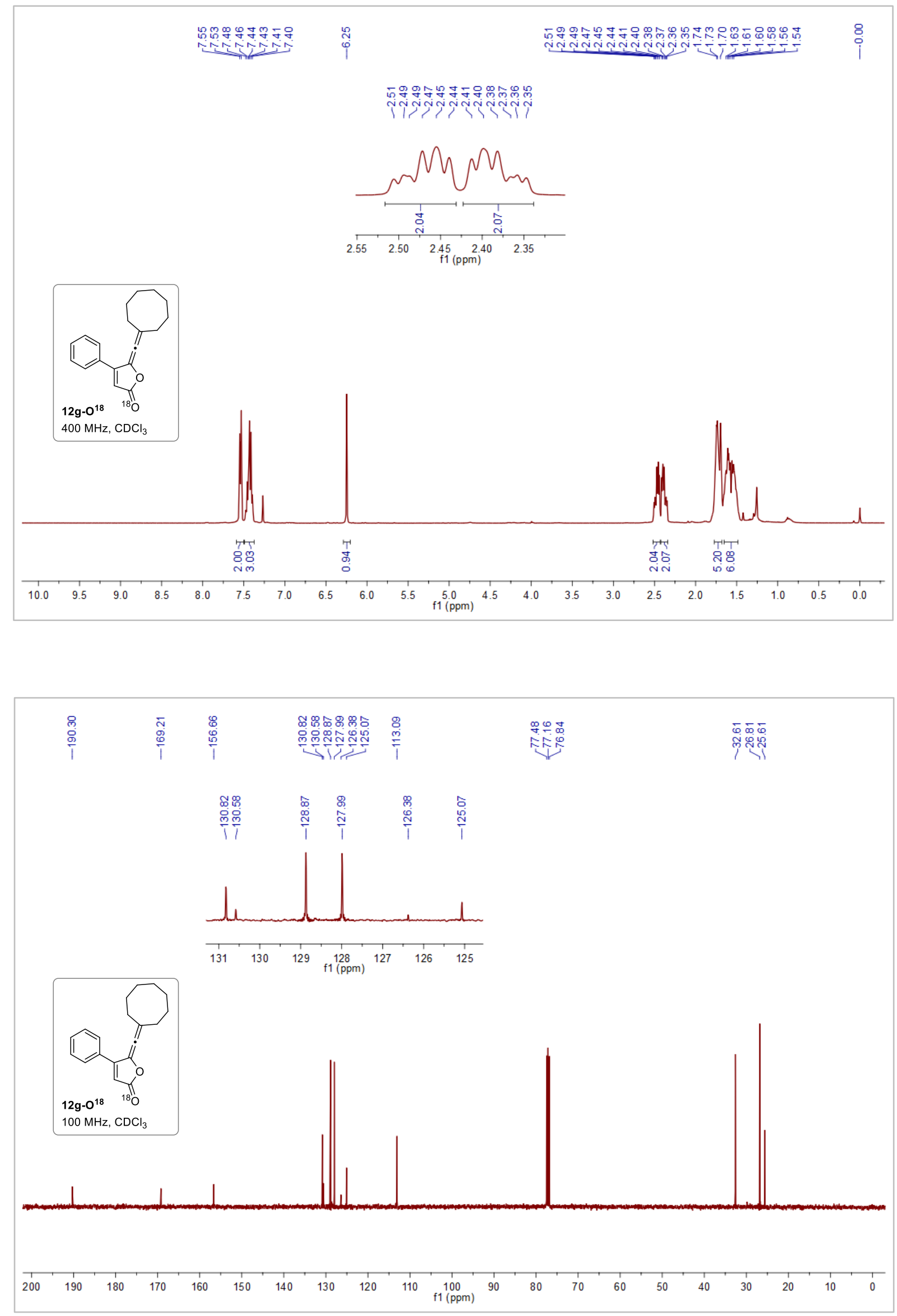
${ }^{1} \mathrm{H}$ NMR $\left(400 \mathrm{MHz}, \mathrm{CDCl}_{3}\right)$ and ${ }^{13} \mathrm{C} \mathrm{NMR}\left(100 \mathrm{MHz}, \mathrm{CDCl}_{3}\right)$ of $\mathbf{1 2 h}$.
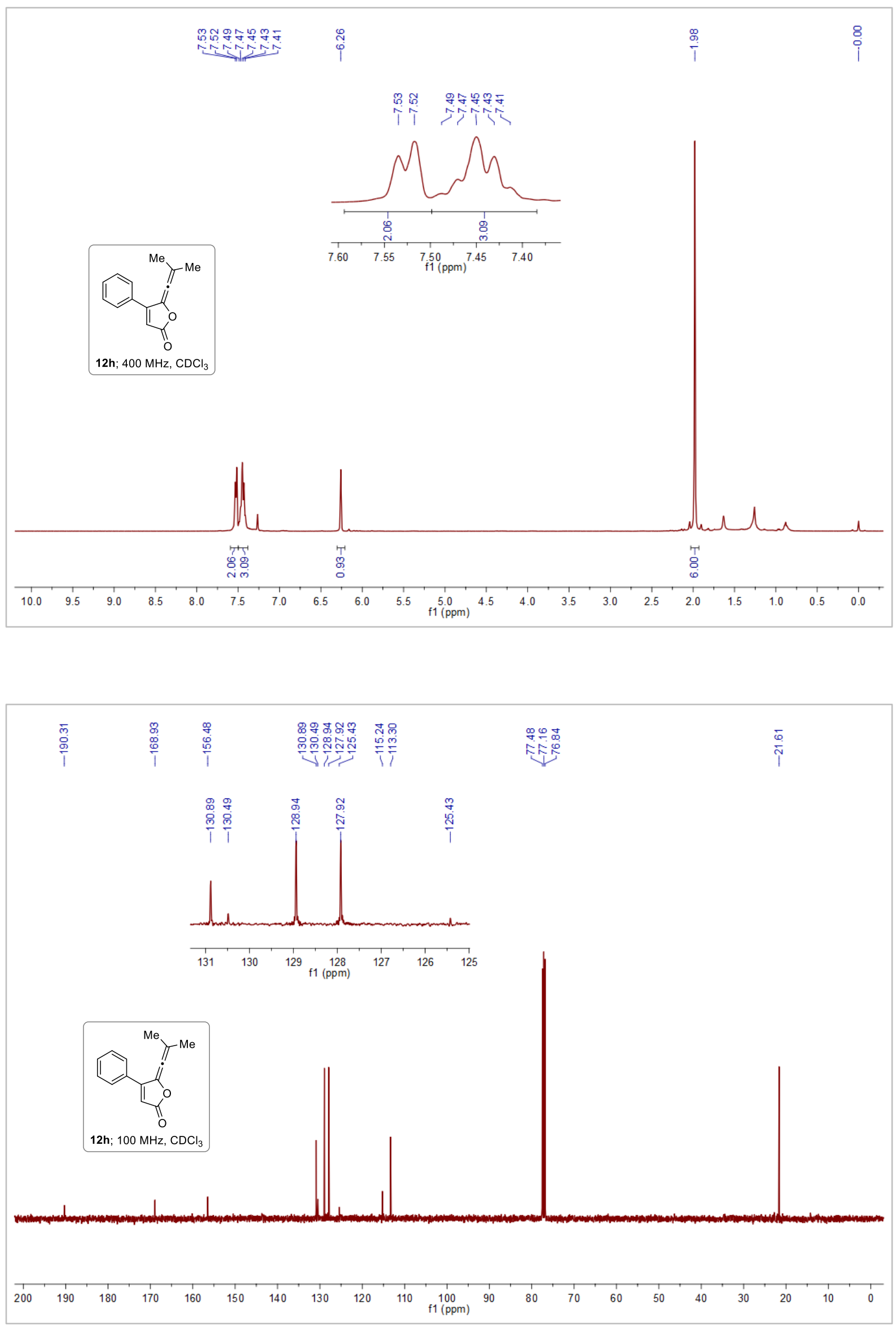
${ }^{1} \mathrm{H}$ NMR $\left(400 \mathrm{MHz}, \mathrm{CDCl}_{3}\right)$ and ${ }^{13} \mathrm{C}$ NMR $\left(100 \mathrm{MHz}, \mathrm{CDCl}_{3}\right)$ of $\mathbf{1 2 i}$.
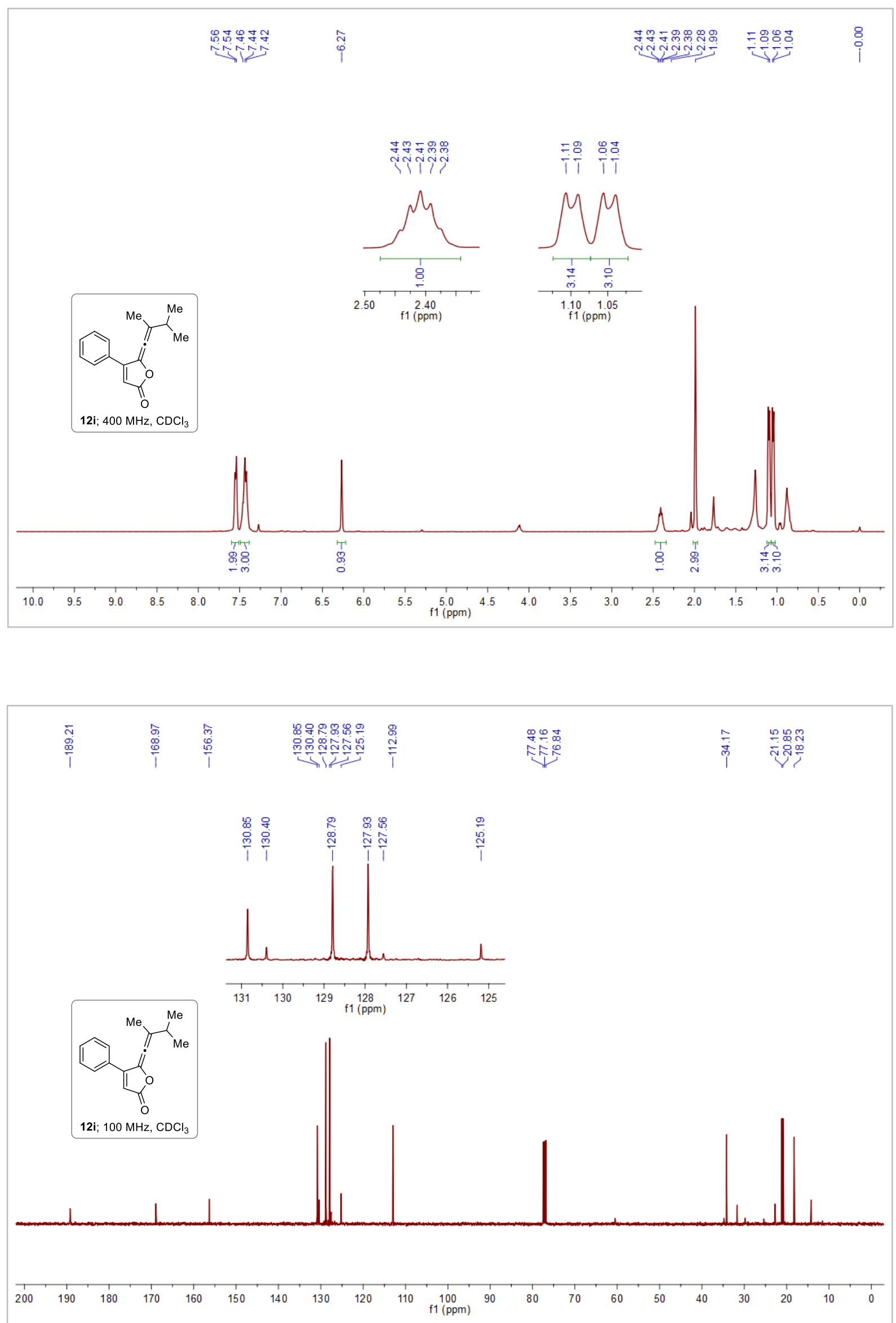
${ }^{1} \mathrm{H}$ NMR $\left(400 \mathrm{MHz}, \mathrm{CDCl}_{3}\right)$ and ${ }^{13} \mathrm{C}$ NMR $\left(100 \mathrm{MHz}, \mathrm{CDCl}_{3}\right)$ of $\mathbf{1 2 j}$.
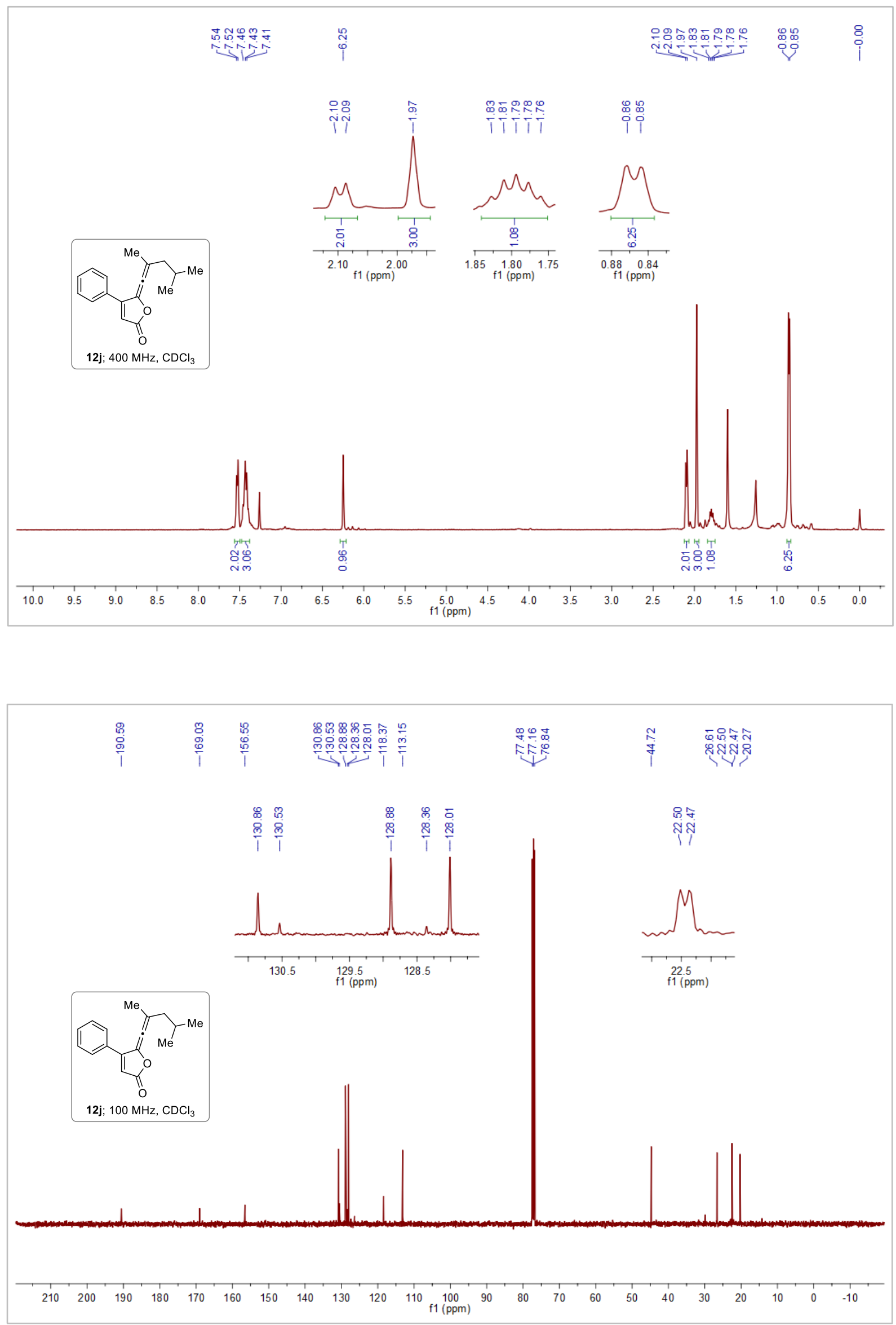
${ }^{1} \mathrm{H}$ NMR (400 MHz, $\left.\mathrm{CDCl}_{3}\right)$ and ${ }^{13} \mathrm{C} \mathrm{NMR}\left(100 \mathrm{MHz}, \mathrm{CDCl}_{3}\right)$ of $\mathbf{1 2 k}$.
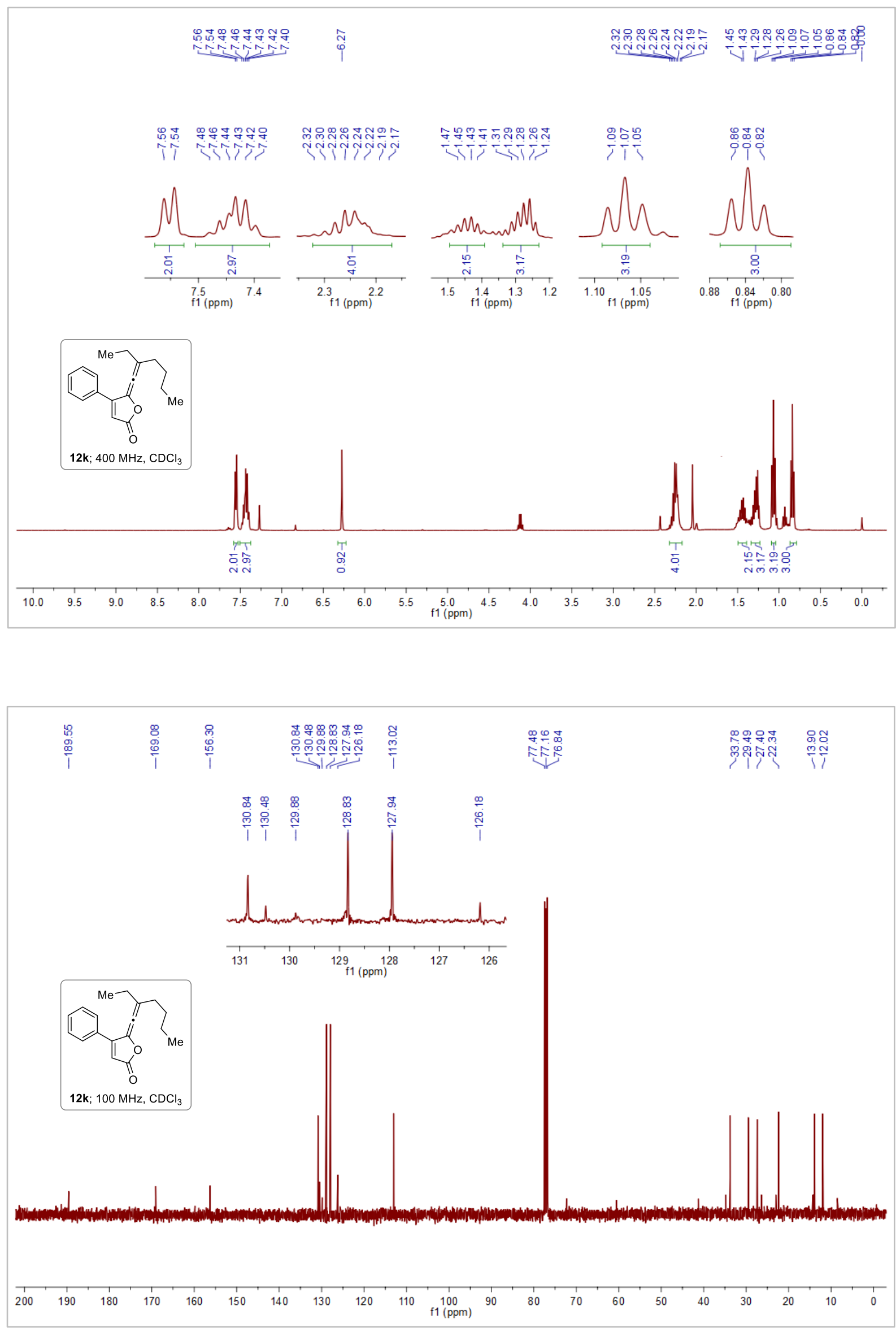
${ }^{1} \mathrm{H}$ NMR $\left(400 \mathrm{MHz}, \mathrm{CDCl}_{3}\right)$ and ${ }^{13} \mathrm{C}$ NMR $\left(100 \mathrm{MHz}, \mathrm{CDCl}_{3}\right)$ of $\mathbf{x x}$.
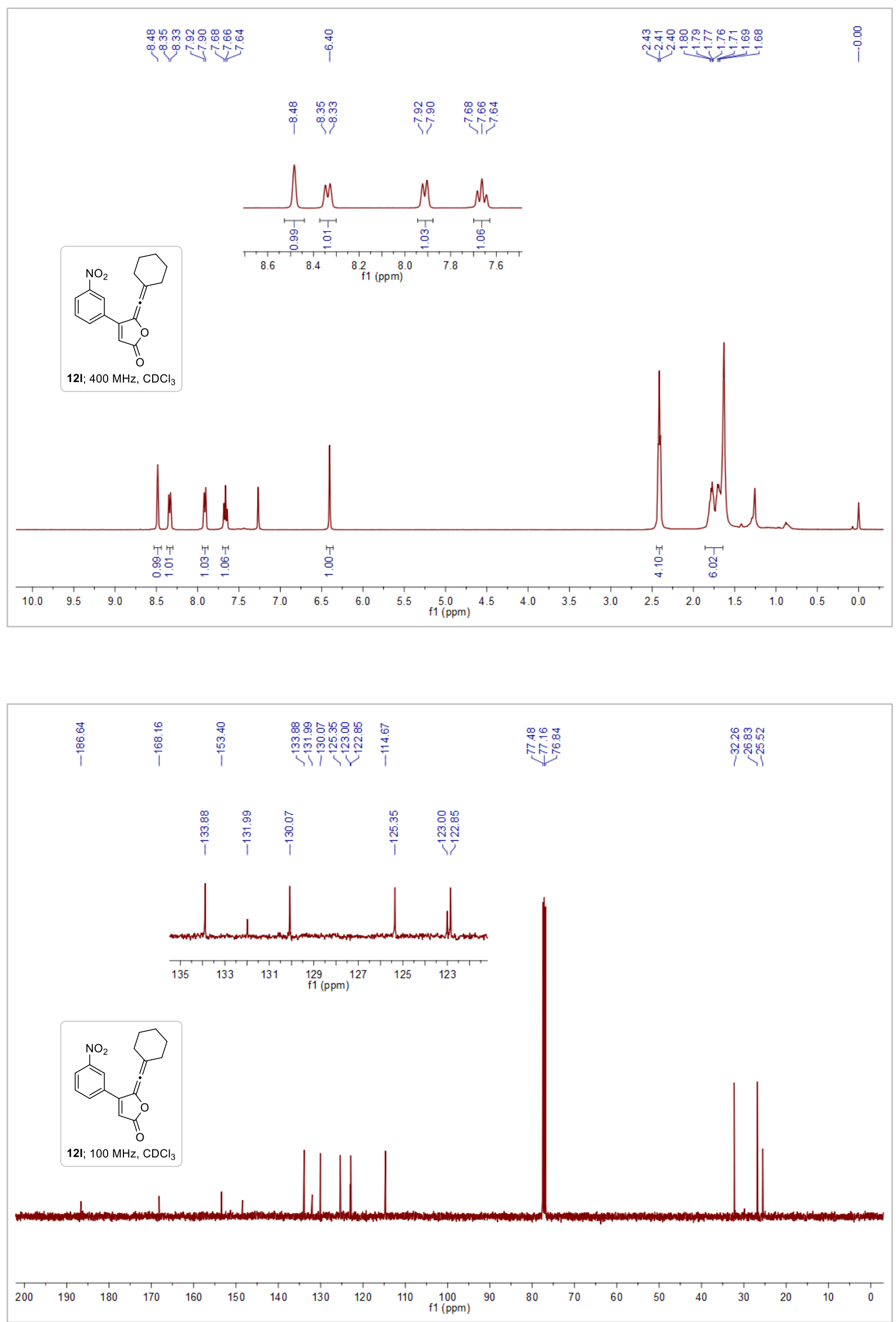
${ }^{1} \mathrm{H}$ NMR $\left(400 \mathrm{MHz}, \mathrm{CDCl}_{3}\right)$ and ${ }^{13} \mathrm{C}$ NMR $\left(100 \mathrm{MHz}, \mathrm{CDCl}_{3}\right)$ of $\mathbf{1 5 a}$.
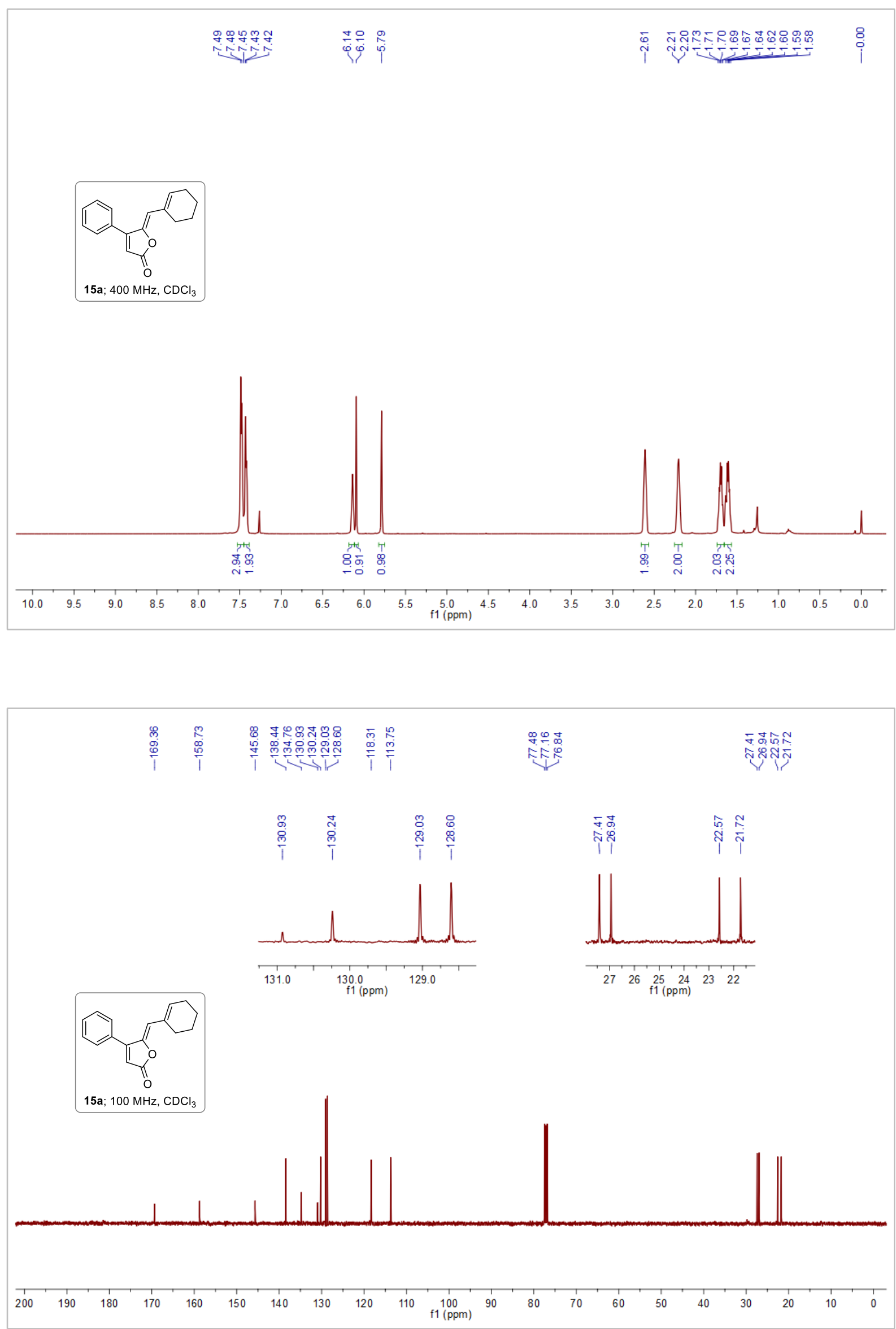
${ }^{1} \mathrm{H}$ NMR $\left(400 \mathrm{MHz}, \mathrm{CDCl}_{3}\right)$ and ${ }^{13} \mathrm{C}$ NMR $\left(100 \mathrm{MHz}, \mathrm{CDCl}_{3}\right)$ of $\mathbf{1 5 e}$.
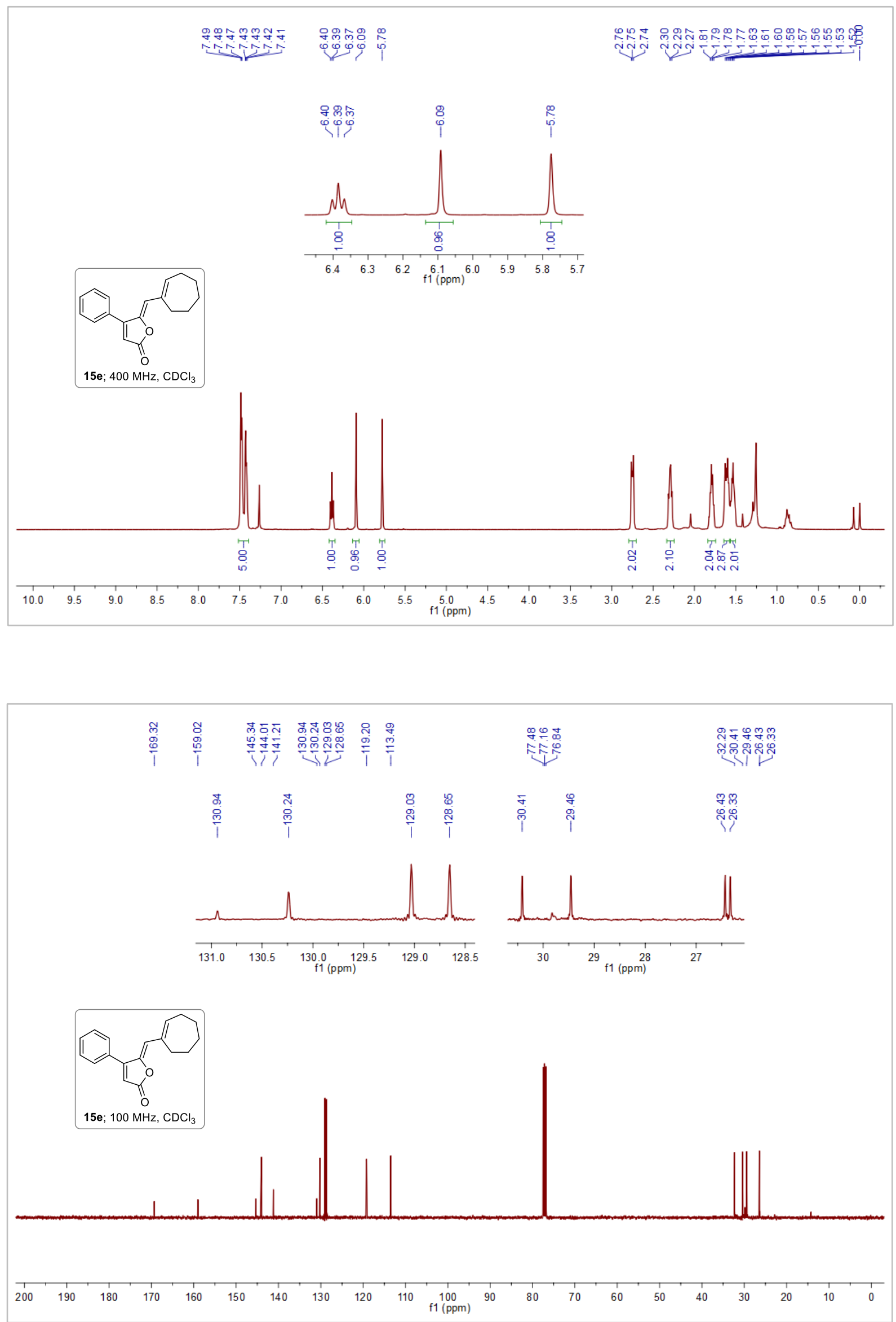
${ }^{1} \mathrm{H}$ NMR (400 MHz, $\left.\mathrm{CDCl}_{3}\right)$ and ${ }^{13} \mathrm{C}$ NMR (100 MHz, $\left.\mathrm{CDCl}_{3}\right)$ of $\mathbf{1 5 f}$.
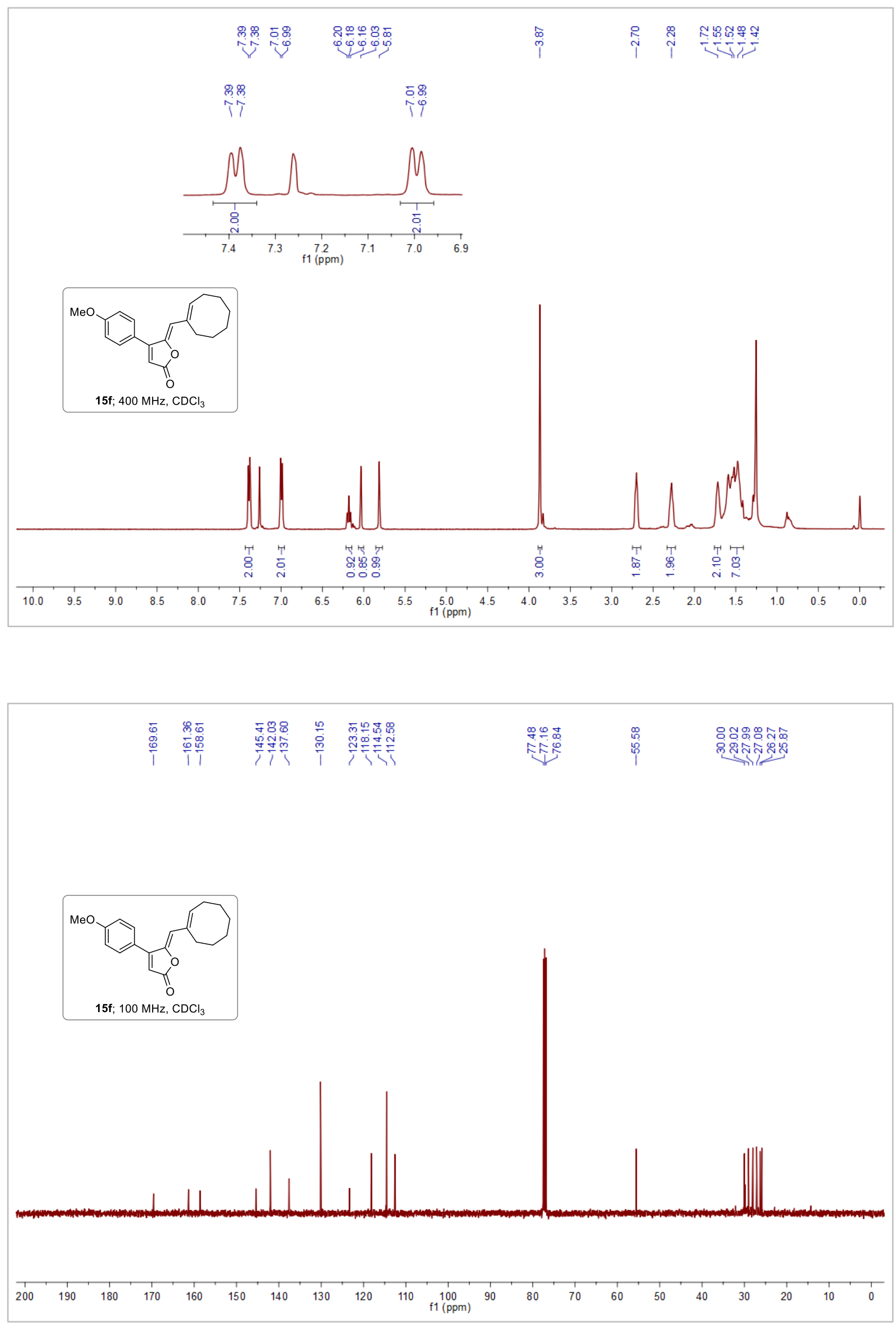
NOESY NMR analysis of $\mathbf{1 5 f}\left(400 \mathrm{MHz}, \mathrm{CDCl}_{3}\right)$

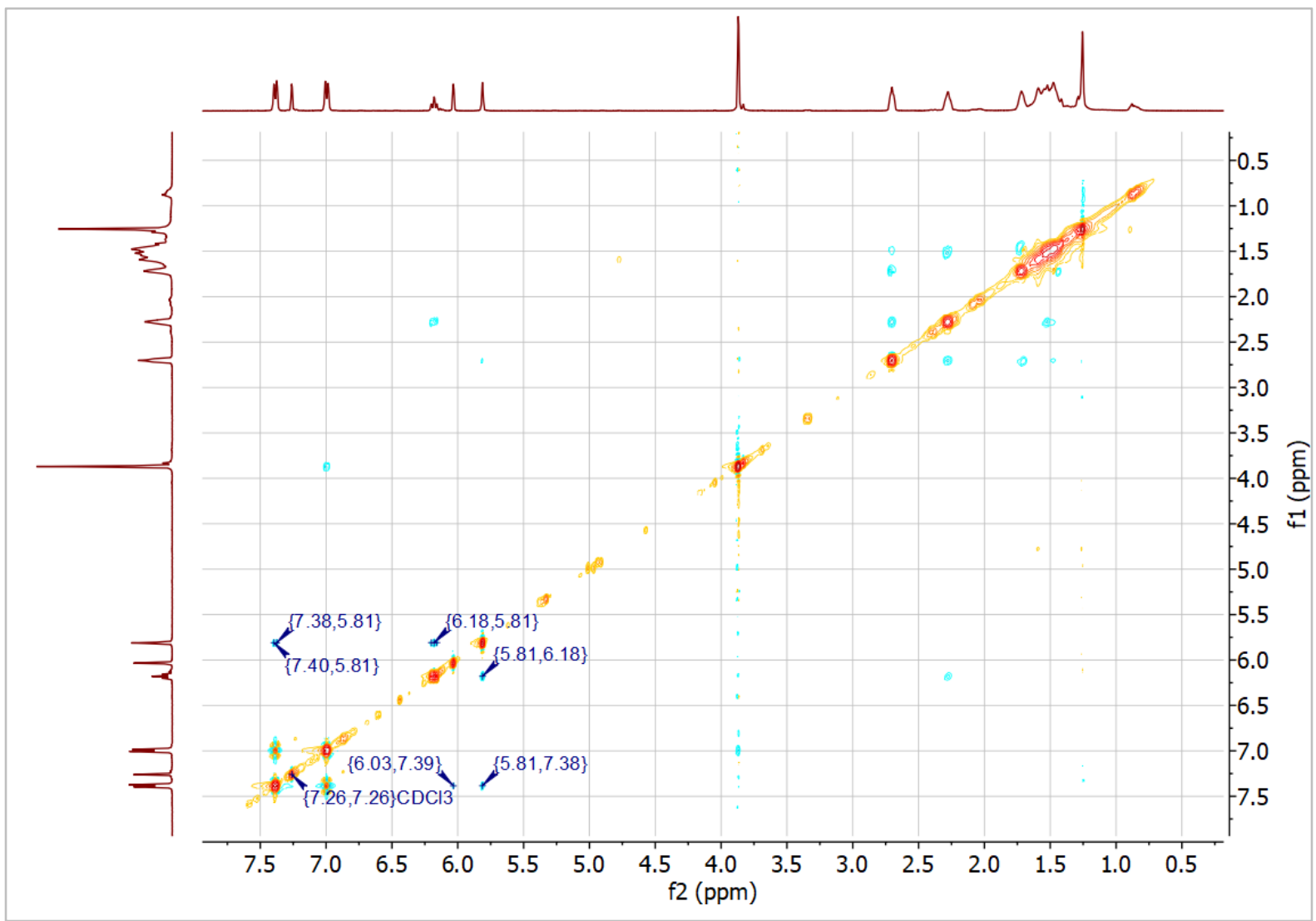

NOESY NMR of $\mathbf{1 5 f}\left(400 \mathrm{MHz}, \mathrm{CDCl}_{3}\right)$ (zoomed)

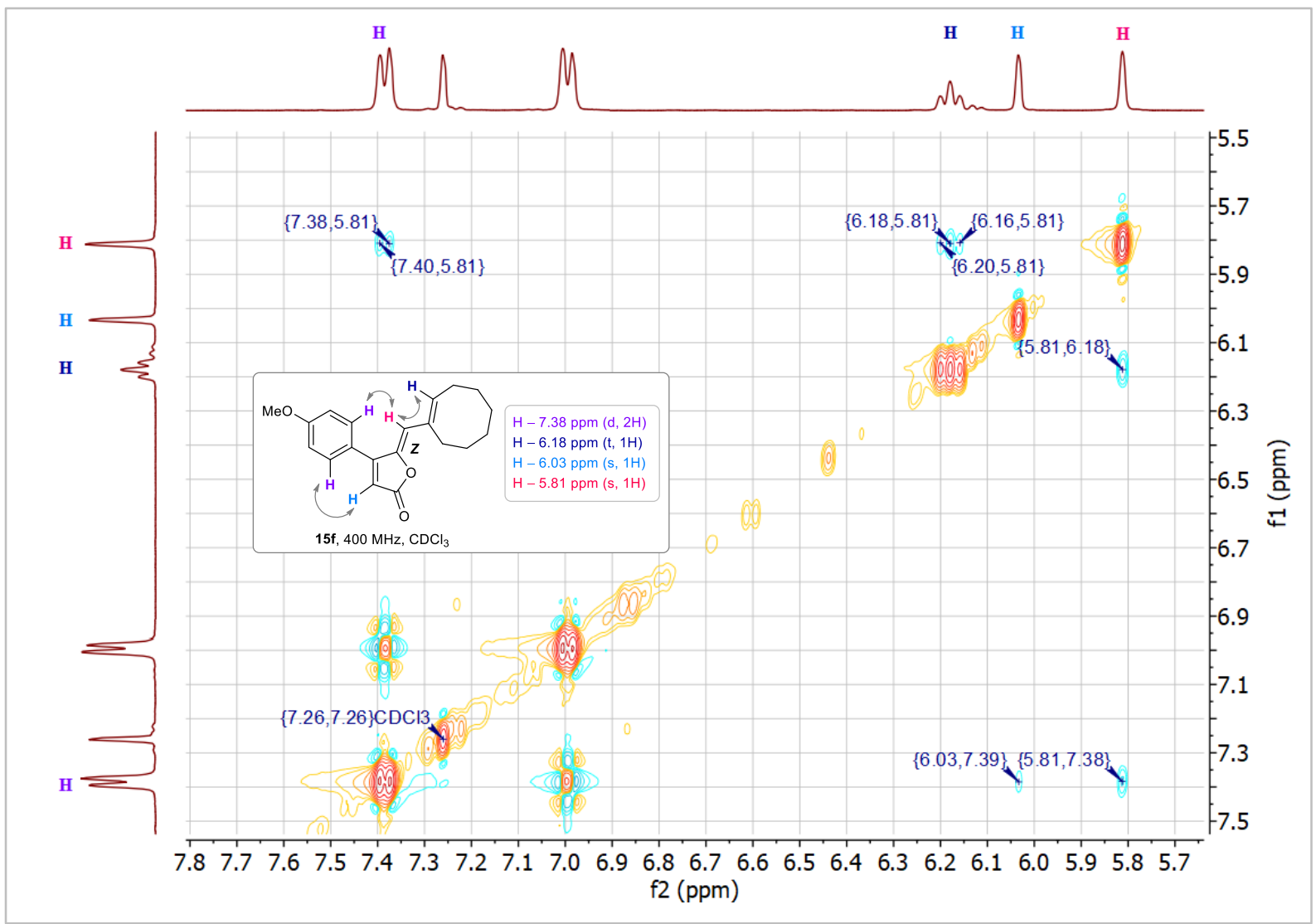


${ }^{1} \mathrm{H}$ NMR (400 MHz, $\left.\mathrm{CDCl}_{3}\right)$ and ${ }^{13} \mathrm{C}$ NMR (100 MHz, $\left.\mathrm{CDCl}_{3}\right)$ of $\mathbf{1 5 g}$.
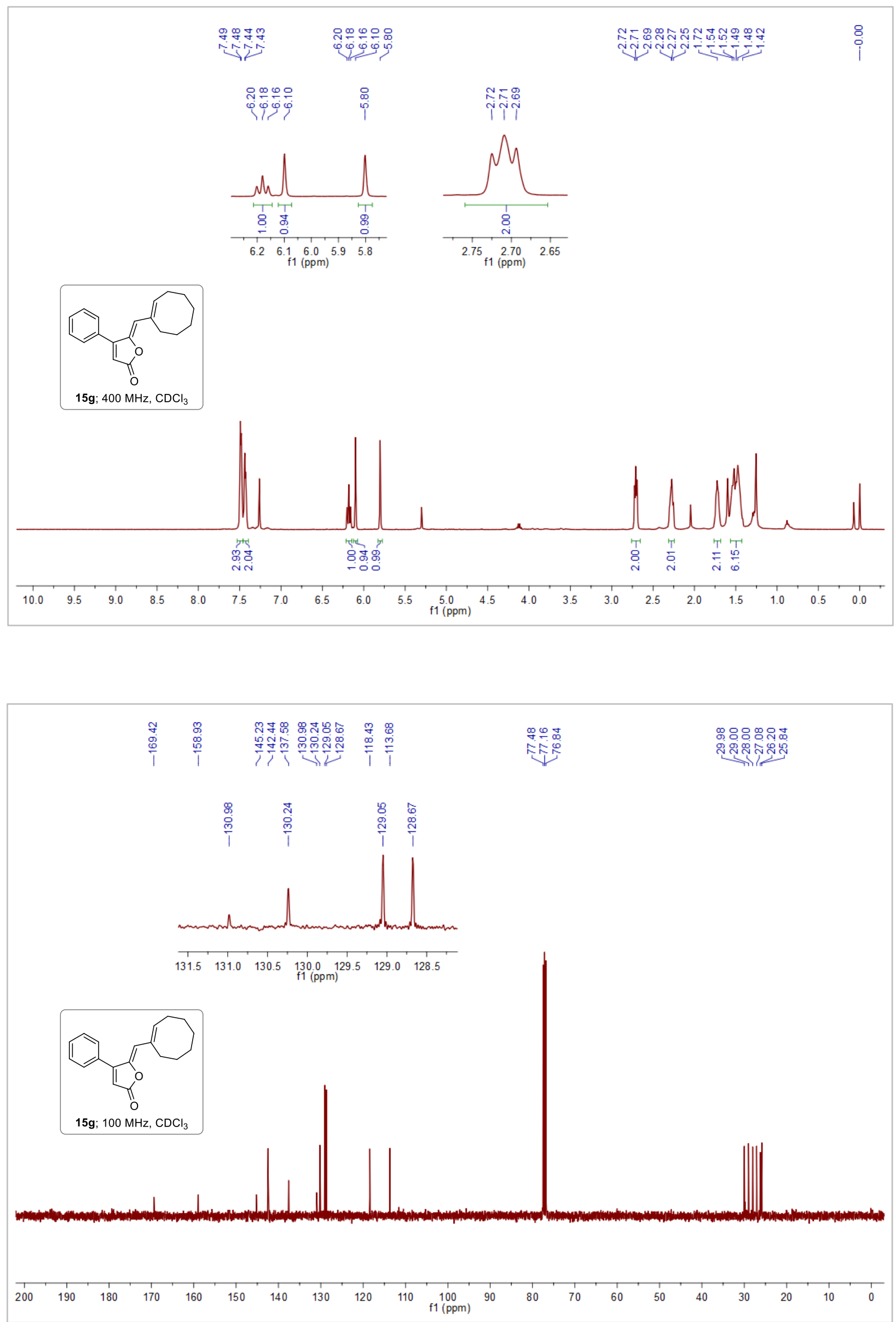
${ }^{1} \mathrm{H}$ NMR $\left(400 \mathrm{MHz}, \mathrm{CDCl}_{3}\right)$ and ${ }^{13} \mathrm{C}$ NMR $\left(100 \mathrm{MHz}, \mathrm{CDCl}_{3}\right)$ of 20.
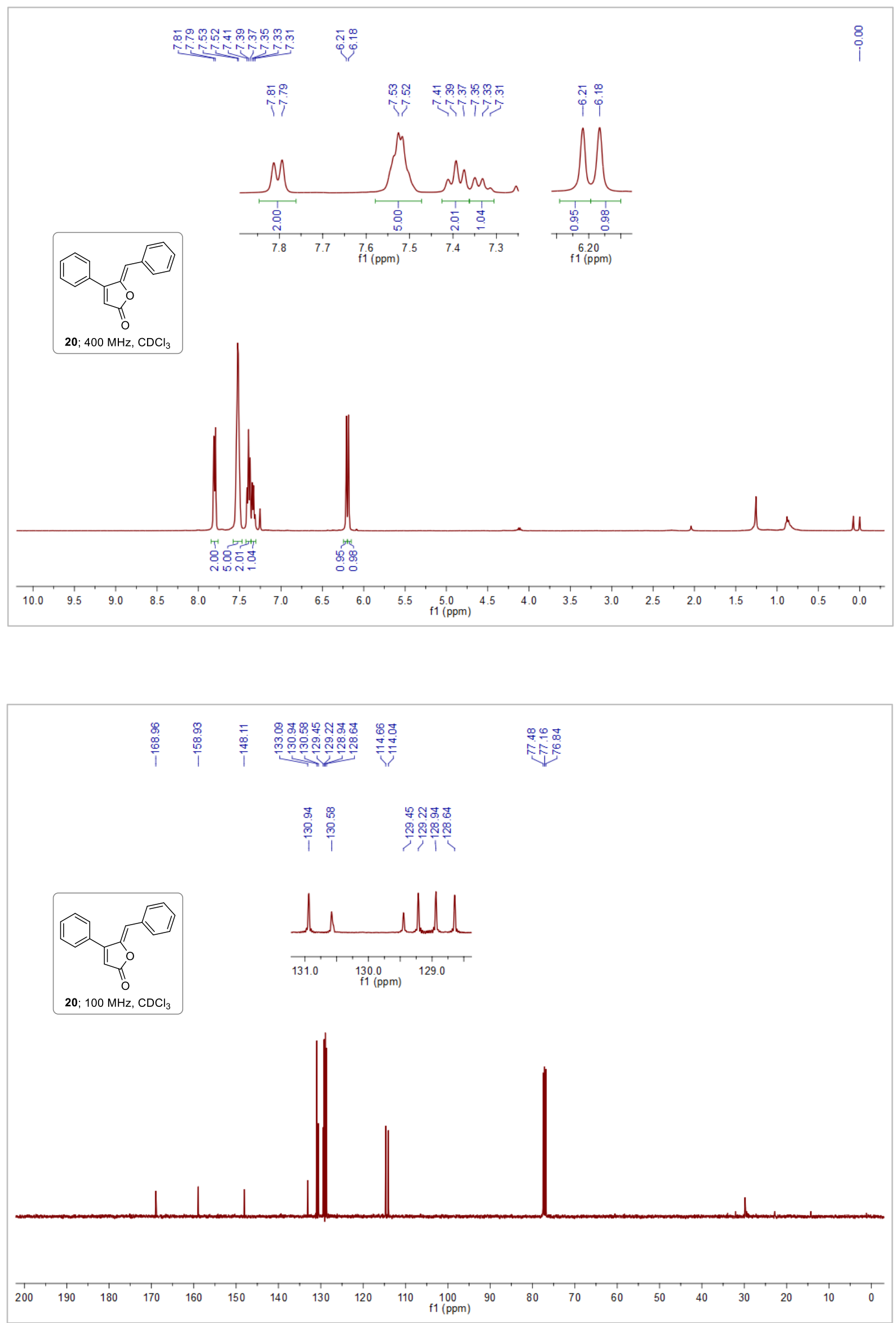
${ }^{1} \mathrm{H}$ NMR $\left(400 \mathrm{MHz}, \mathrm{CDCl}_{3}\right)$ and ${ }^{13} \mathrm{C}$ NMR $\left(100 \mathrm{MHz}, \mathrm{CDCl}_{3}\right)$ of 21.
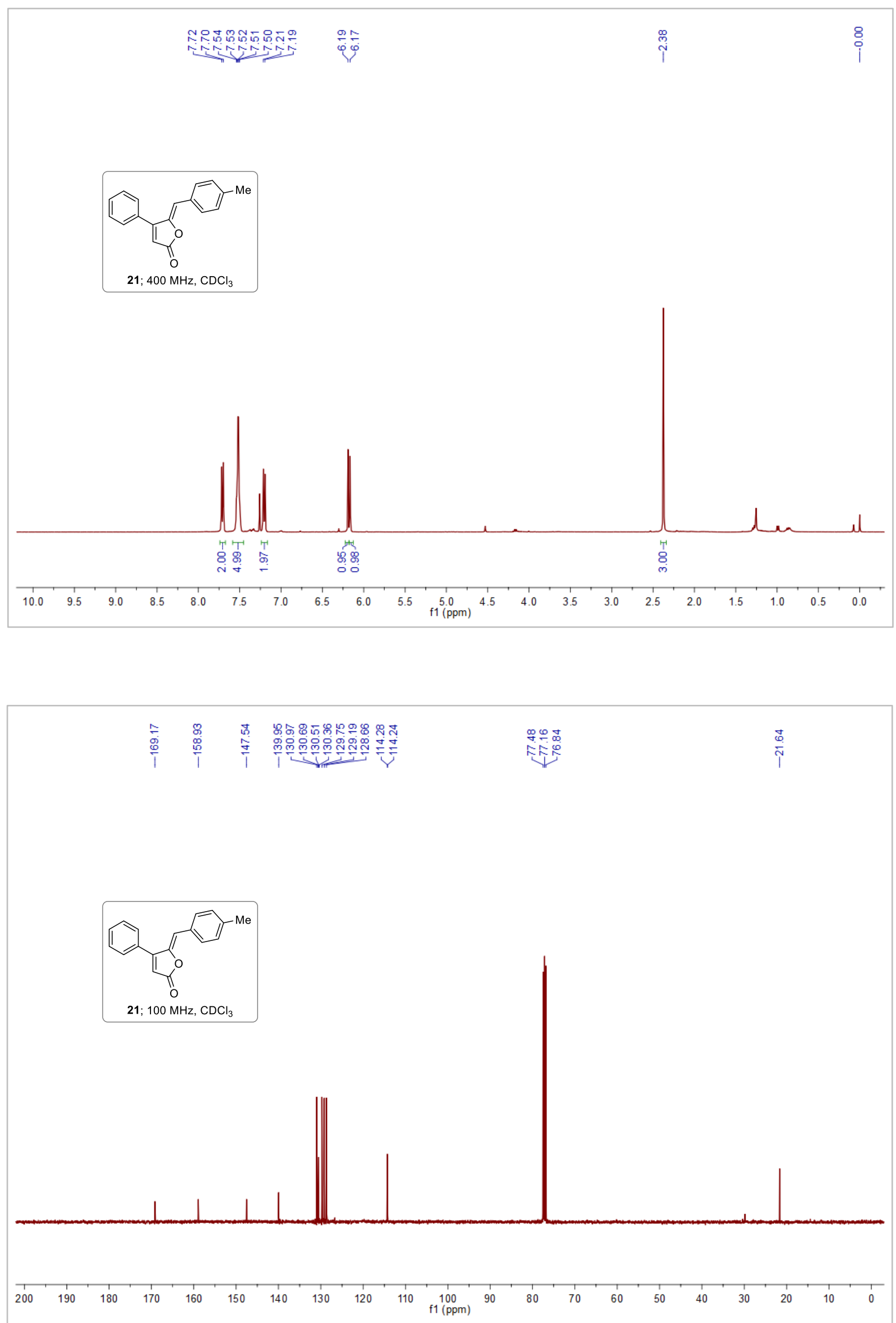
${ }^{1} \mathrm{H}$ NMR $\left(400 \mathrm{MHz}, \mathrm{CDCl}_{3}\right)$ and ${ }^{13} \mathrm{C}$ NMR $\left(100 \mathrm{MHz}, \mathrm{CDCl}_{3}\right)$ of 22.
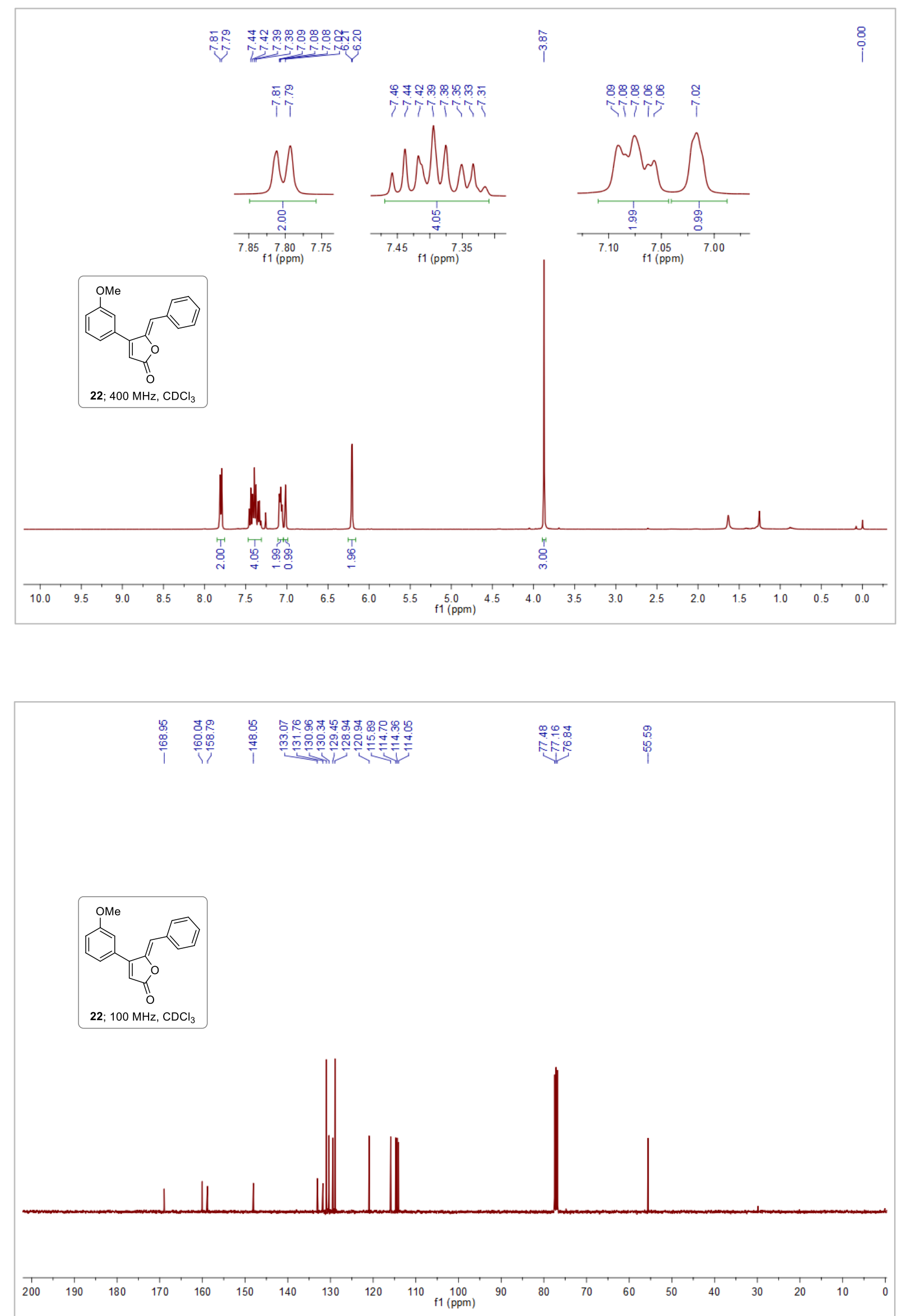
${ }^{1} \mathrm{H}$ NMR $\left(400 \mathrm{MHz}, \mathrm{CDCl}_{3}\right)$ and ${ }^{13} \mathrm{C} \mathrm{NMR}\left(100 \mathrm{MHz}, \mathrm{CDCl}_{3}\right)$ of 27.
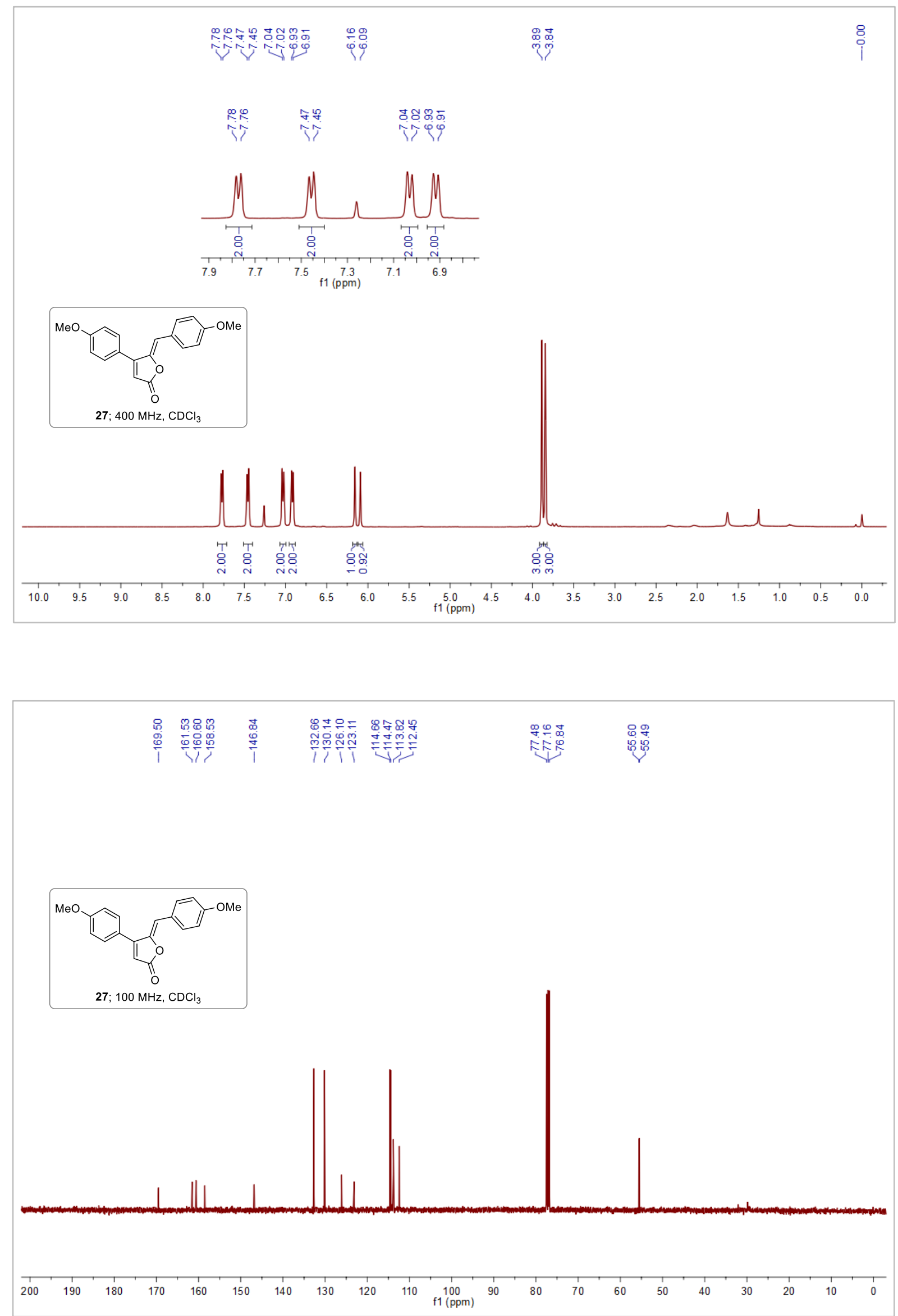

27; $100 \mathrm{MHz}, \mathrm{CDCl}_{3}$ 
${ }^{1} \mathrm{H}$ NMR (400 MHz, DMSO- $\left.d_{6}\right)$ and ${ }^{13} \mathrm{C}$ NMR (100 MHz, DMSO- $\left.d_{6}\right)$ of Rubrolide E.
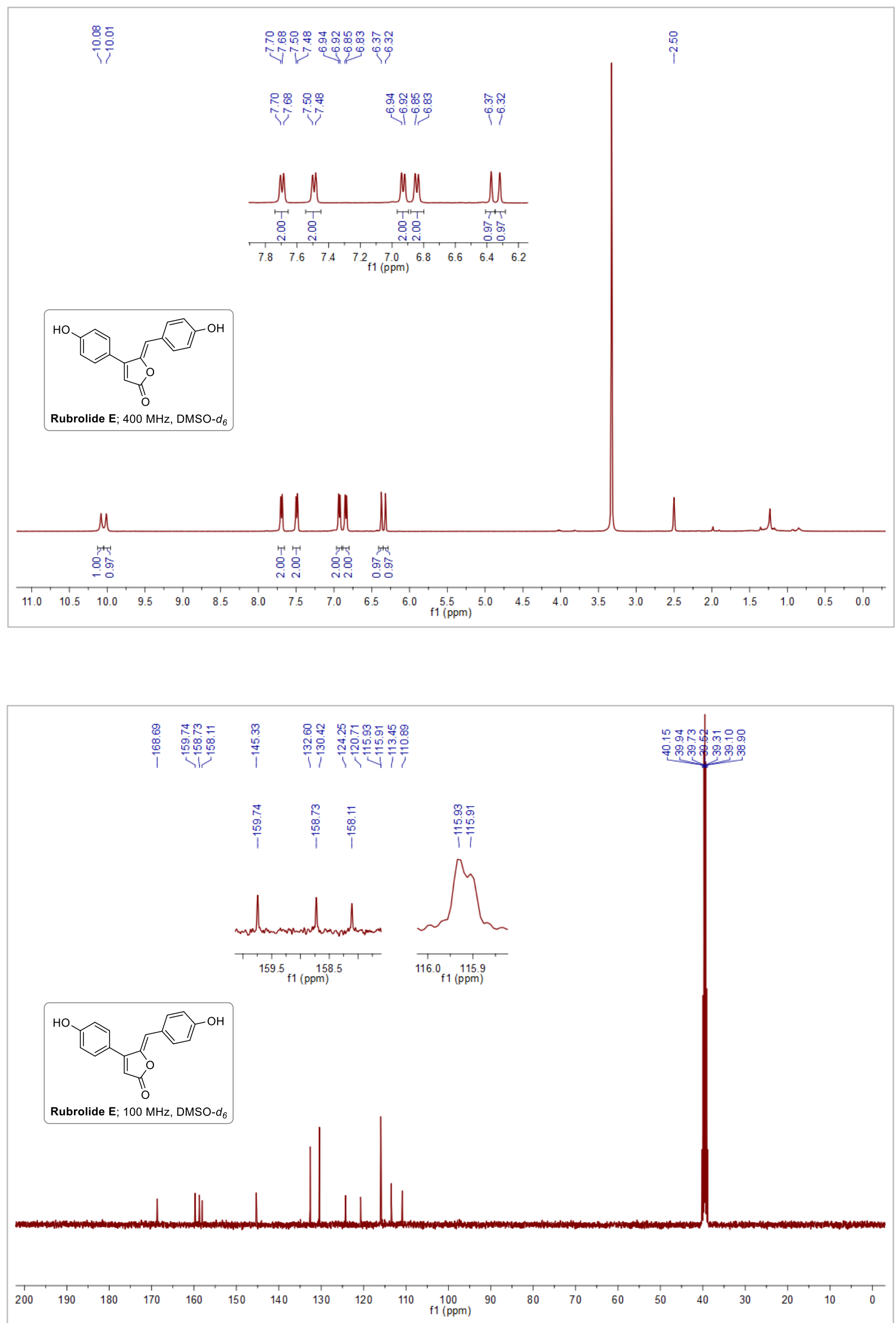
${ }^{1} \mathrm{H}$ NMR $\left(400 \mathrm{MHz}, \mathrm{CDCl}_{3}\right)$ and ${ }^{13} \mathrm{C} \mathrm{NMR}\left(100 \mathrm{MHz}, \mathrm{CDCl}_{3}\right)$ of $9 \mathbf{a}$.
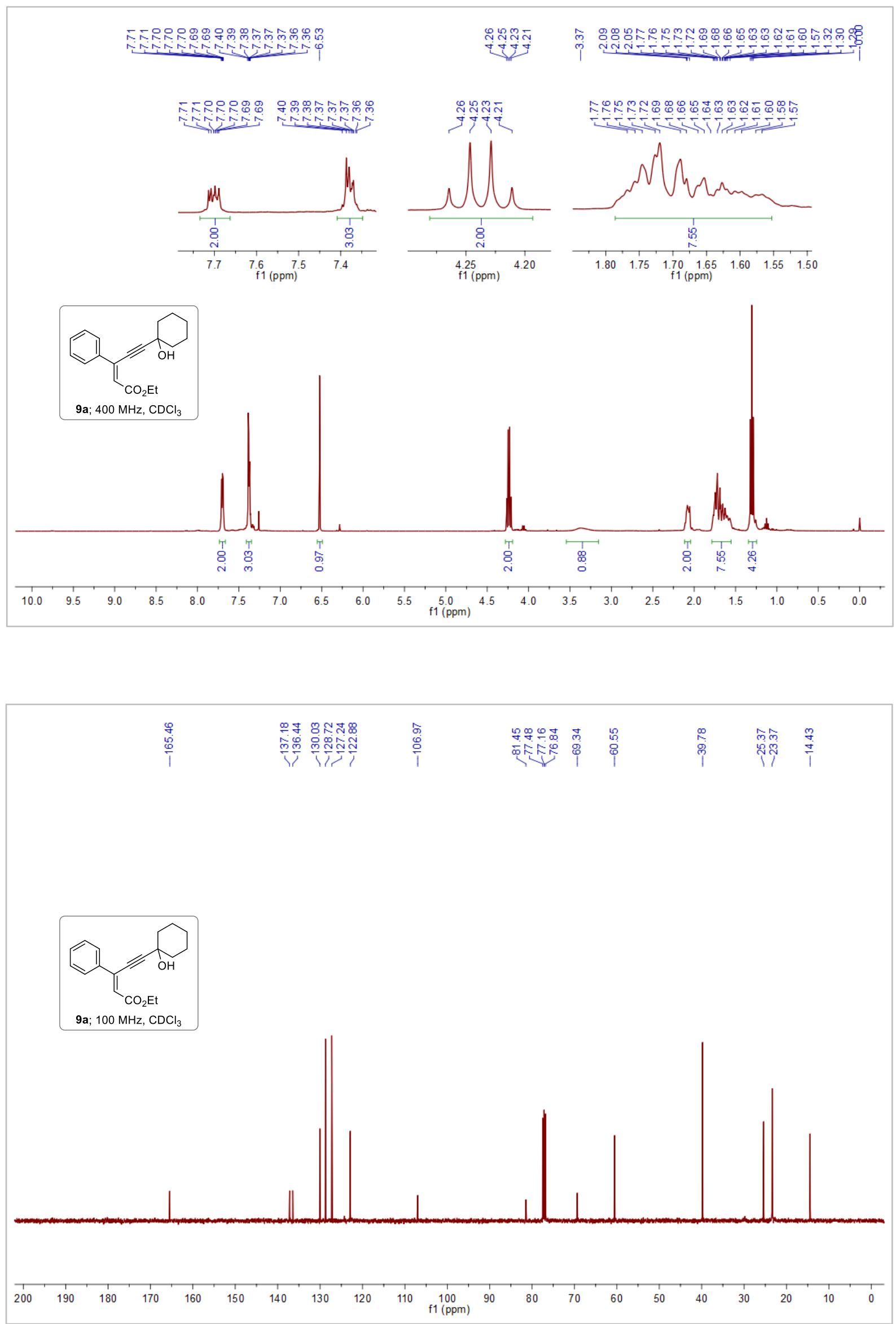
${ }^{1} \mathrm{H}$ NMR $\left(400 \mathrm{MHz}, \mathrm{CDCl}_{3}\right)$ and ${ }^{13} \mathrm{C} \mathrm{NMR}\left(100 \mathrm{MHz}, \mathrm{CDCl}_{3}\right)$ of $\mathbf{9 b}$.
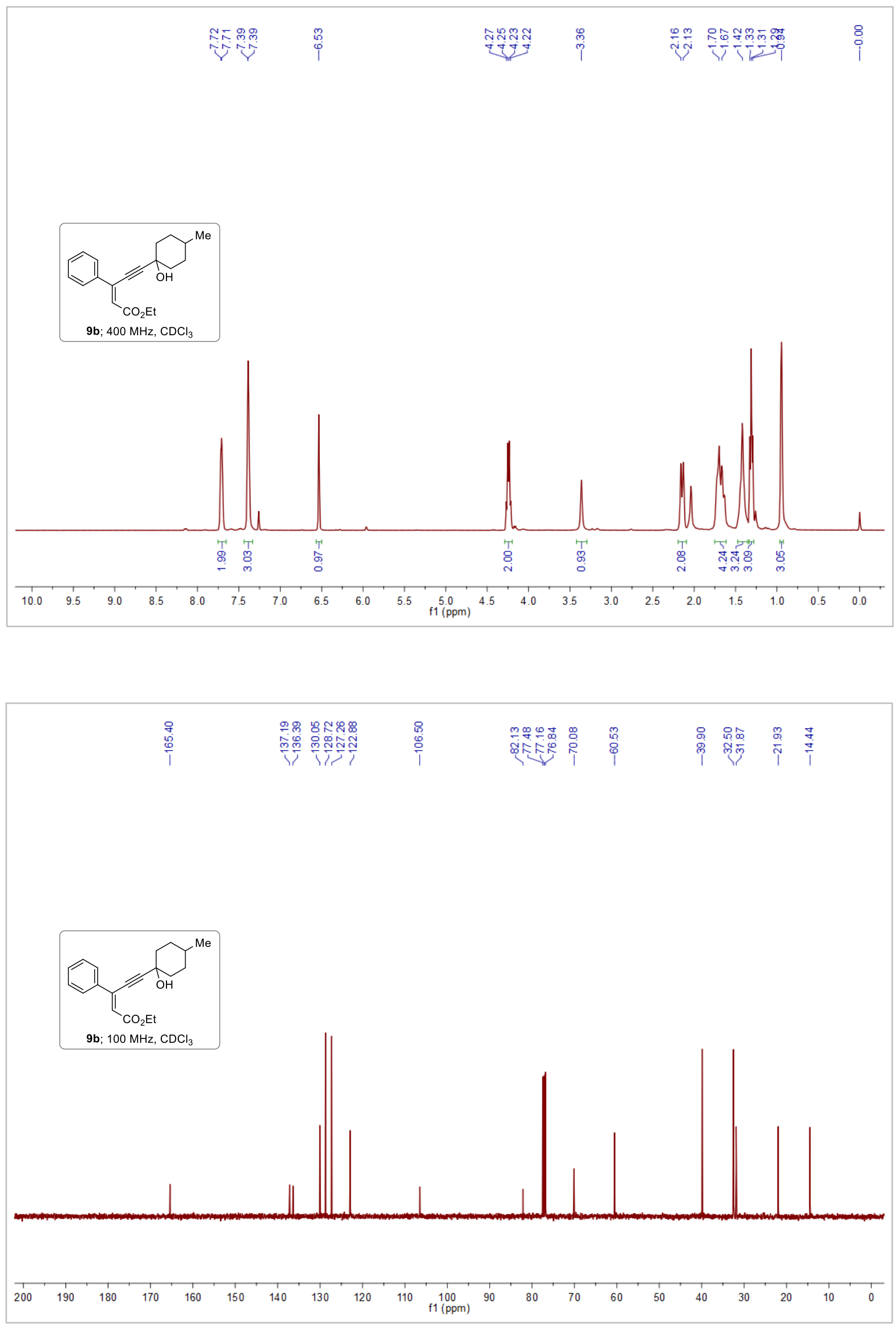
${ }^{1} \mathrm{H} \mathrm{NMR}\left(400 \mathrm{MHz}, \mathrm{CDCl}_{3}\right)$ and ${ }^{13} \mathrm{C} \mathrm{NMR}\left(100 \mathrm{MHz}, \mathrm{CDCl}_{3}\right)$ of $9 \mathbf{c}$.
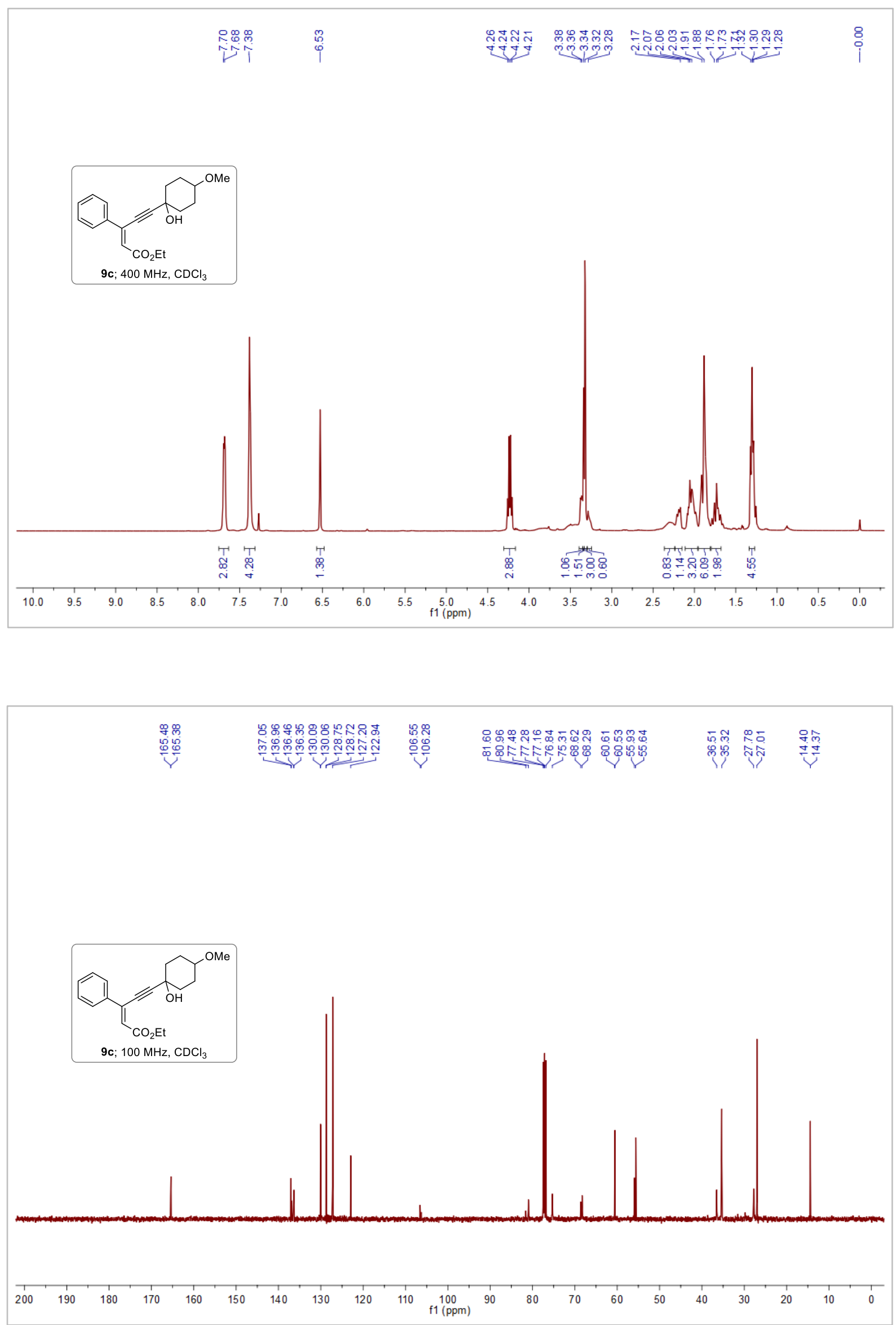
${ }^{1} \mathrm{H}$ NMR $\left(400 \mathrm{MHz}, \mathrm{CDCl}_{3}\right)$ and ${ }^{13} \mathrm{C} \mathrm{NMR}\left(100 \mathrm{MHz}, \mathrm{CDCl}_{3}\right)$ of $9 d$.
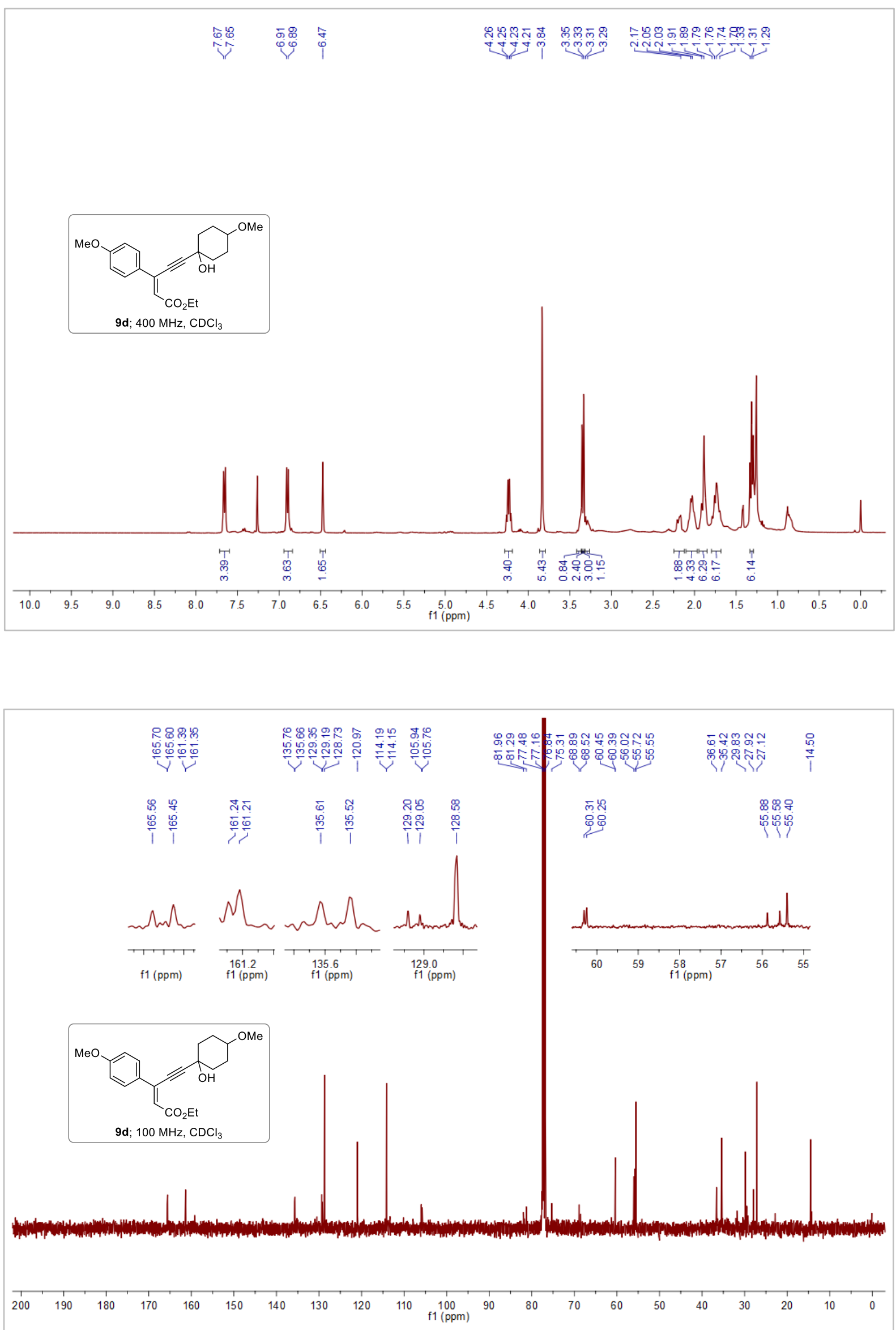
${ }^{1} \mathrm{H}$ NMR $\left(400 \mathrm{MHz}, \mathrm{CDCl}_{3}\right)$ and ${ }^{13} \mathrm{C} \mathrm{NMR}\left(100 \mathrm{MHz}, \mathrm{CDCl}_{3}\right)$ of $9 \mathrm{e}$.
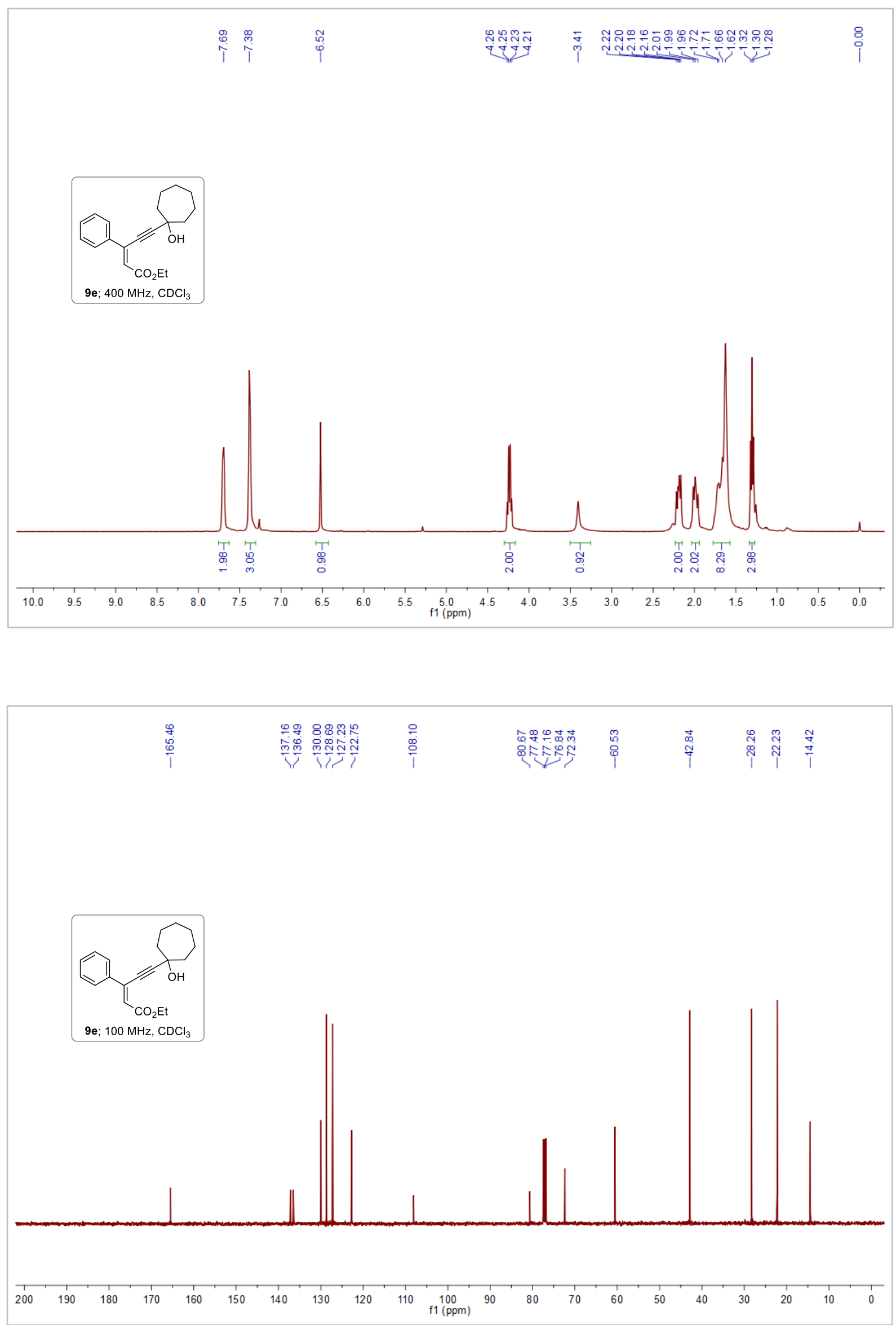
${ }^{1} \mathrm{H}$ NMR $\left(400 \mathrm{MHz}, \mathrm{CDCl}_{3}\right)$ and ${ }^{13} \mathrm{C} \mathrm{NMR}\left(100 \mathrm{MHz}, \mathrm{CDCl}_{3}\right)$ of $9 f$.

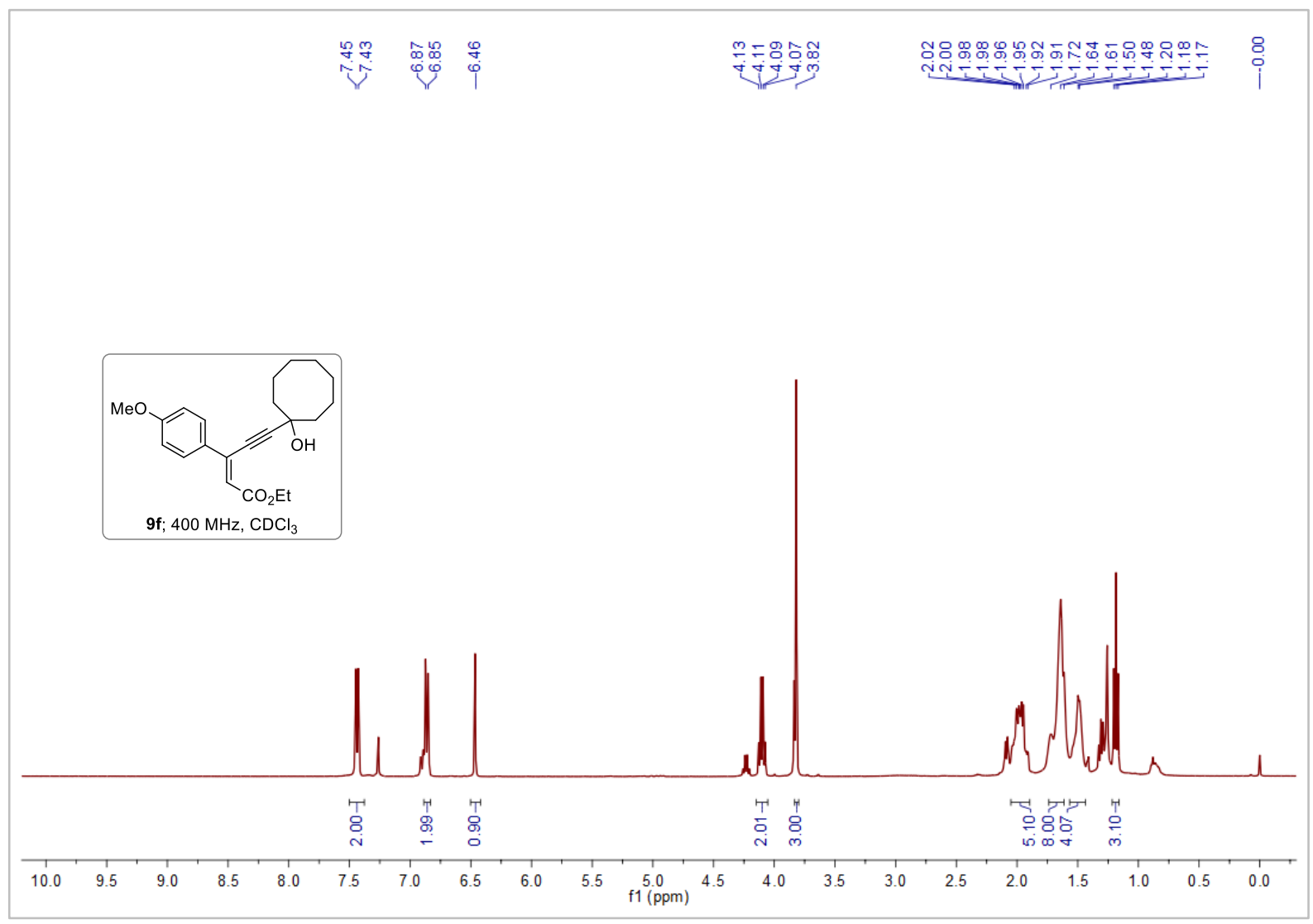

\section{$\begin{array}{ll}1 & 1 \\ 0 & 0 \\ 0 & 0 \\ 0 & 0 \\ 0 & 0\end{array}$

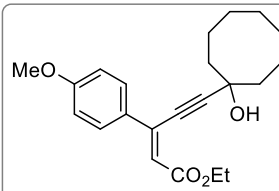

9f; $100 \mathrm{MHz}, \mathrm{CDCl}_{3}$

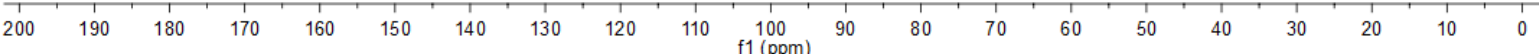


${ }^{1} \mathrm{H}$ NMR (400 MHz, $\left.\mathrm{CDCl}_{3}\right)$ and ${ }^{13} \mathrm{C} \mathrm{NMR}\left(100 \mathrm{MHz}, \mathrm{CDCl}_{3}\right)$ of 9g.
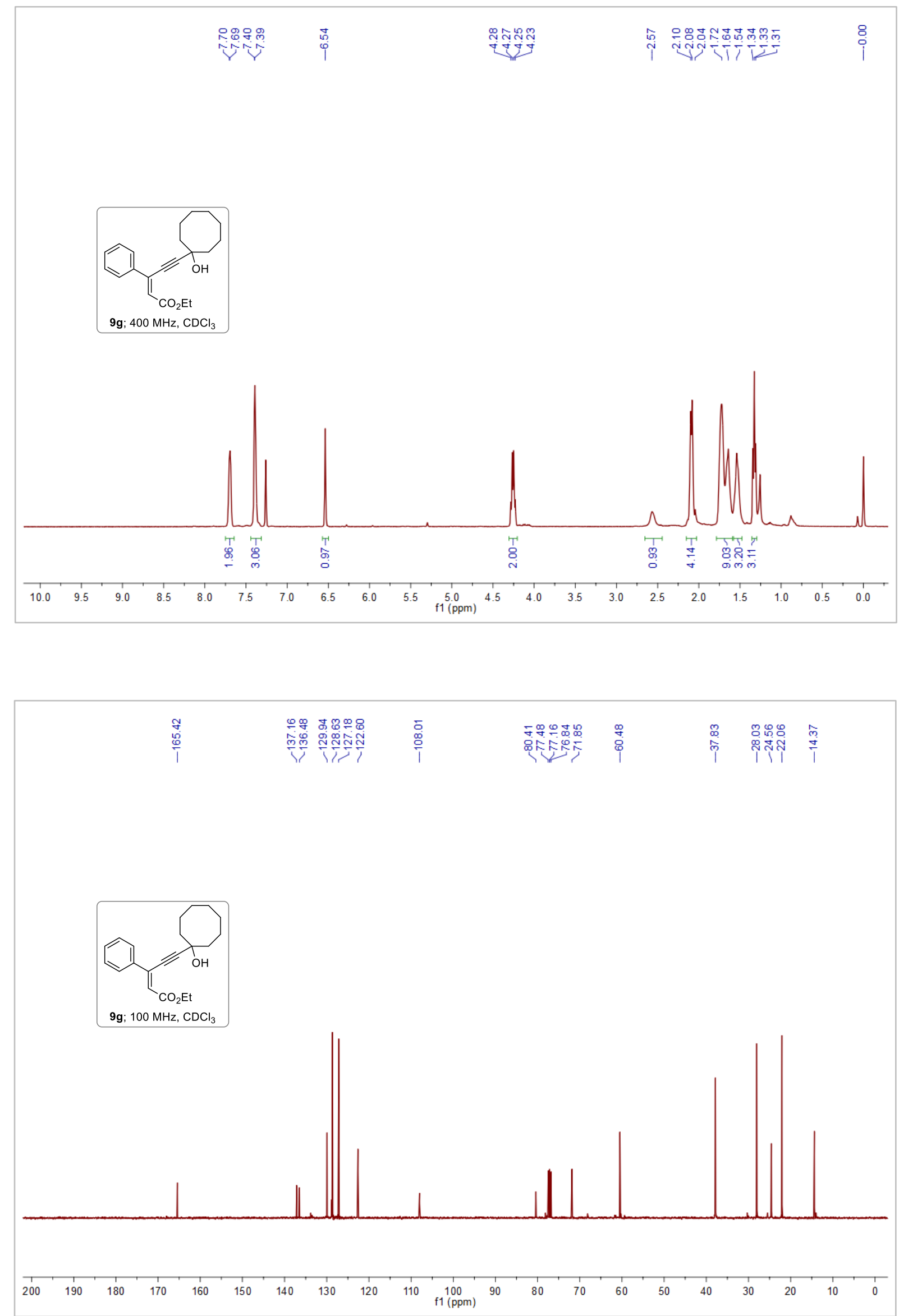
${ }^{1} \mathrm{H}$ NMR $\left(400 \mathrm{MHz}, \mathrm{CDCl}_{3}\right)$ and ${ }^{13} \mathrm{C} \mathrm{NMR}\left(100 \mathrm{MHz}, \mathrm{CDCl}_{3}\right)$ of $\mathbf{9 h}$.
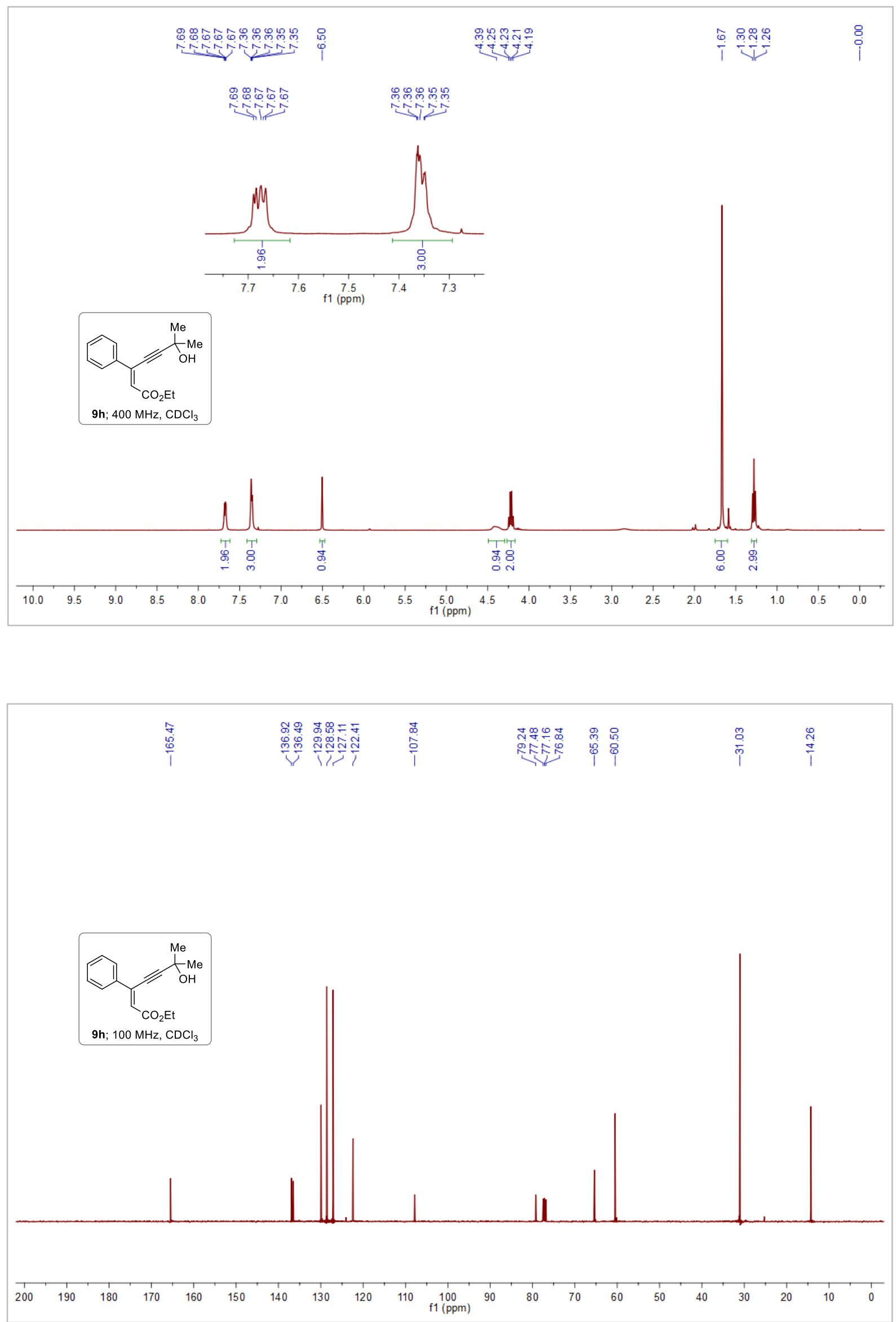
${ }^{1} \mathrm{H}$ NMR $\left(400 \mathrm{MHz}, \mathrm{CDCl}_{3}\right)$ and ${ }^{13} \mathrm{C}$ NMR $\left(100 \mathrm{MHz}, \mathrm{CDCl}_{3}\right)$ of $9 \mathbf{i}$.
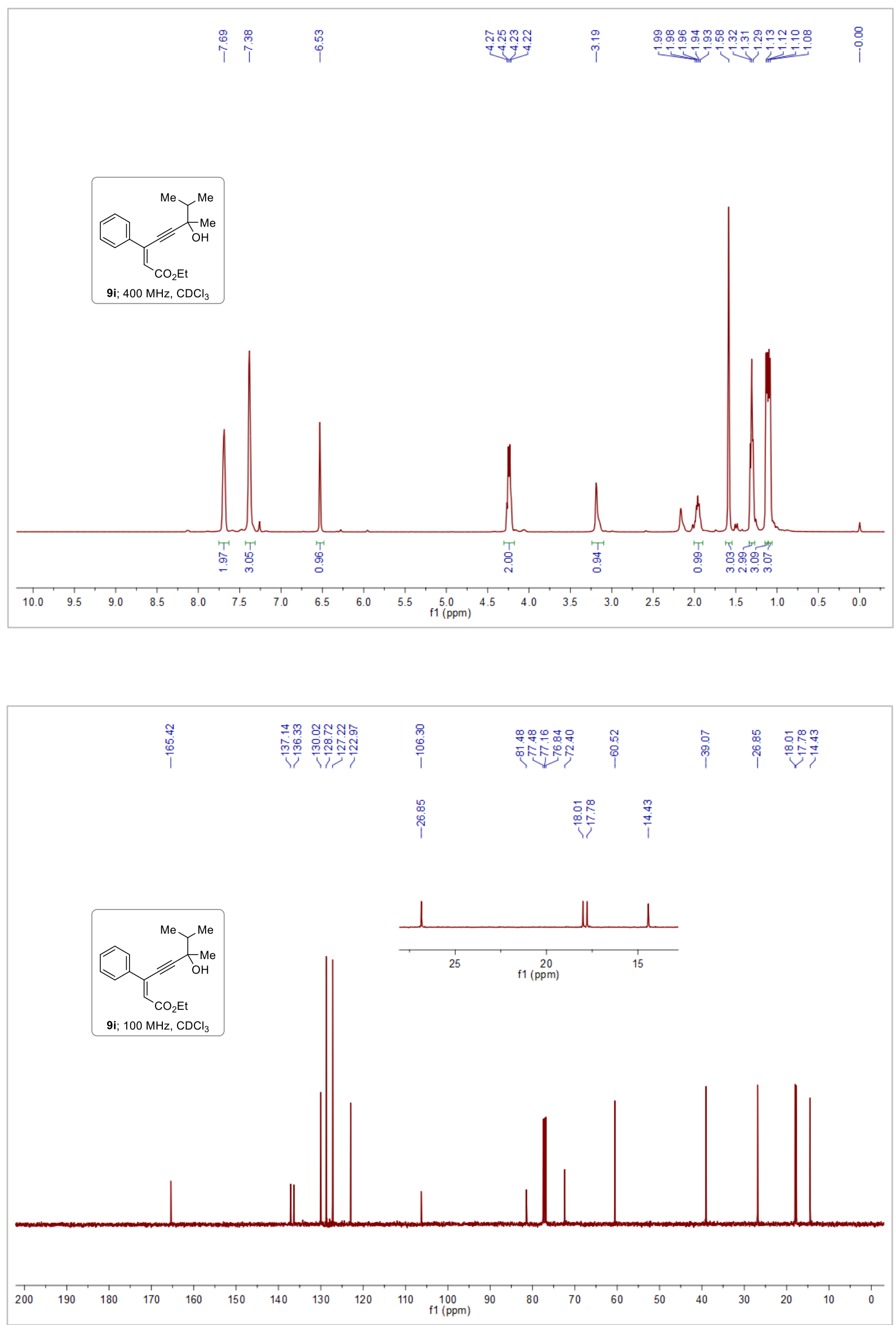
${ }^{1} \mathrm{H}$ NMR $\left(400 \mathrm{MHz}, \mathrm{CDCl}_{3}\right)$ and ${ }^{13} \mathrm{C} \mathrm{NMR}\left(100 \mathrm{MHz}, \mathrm{CDCl}_{3}\right)$ of $\mathbf{9 j}$.
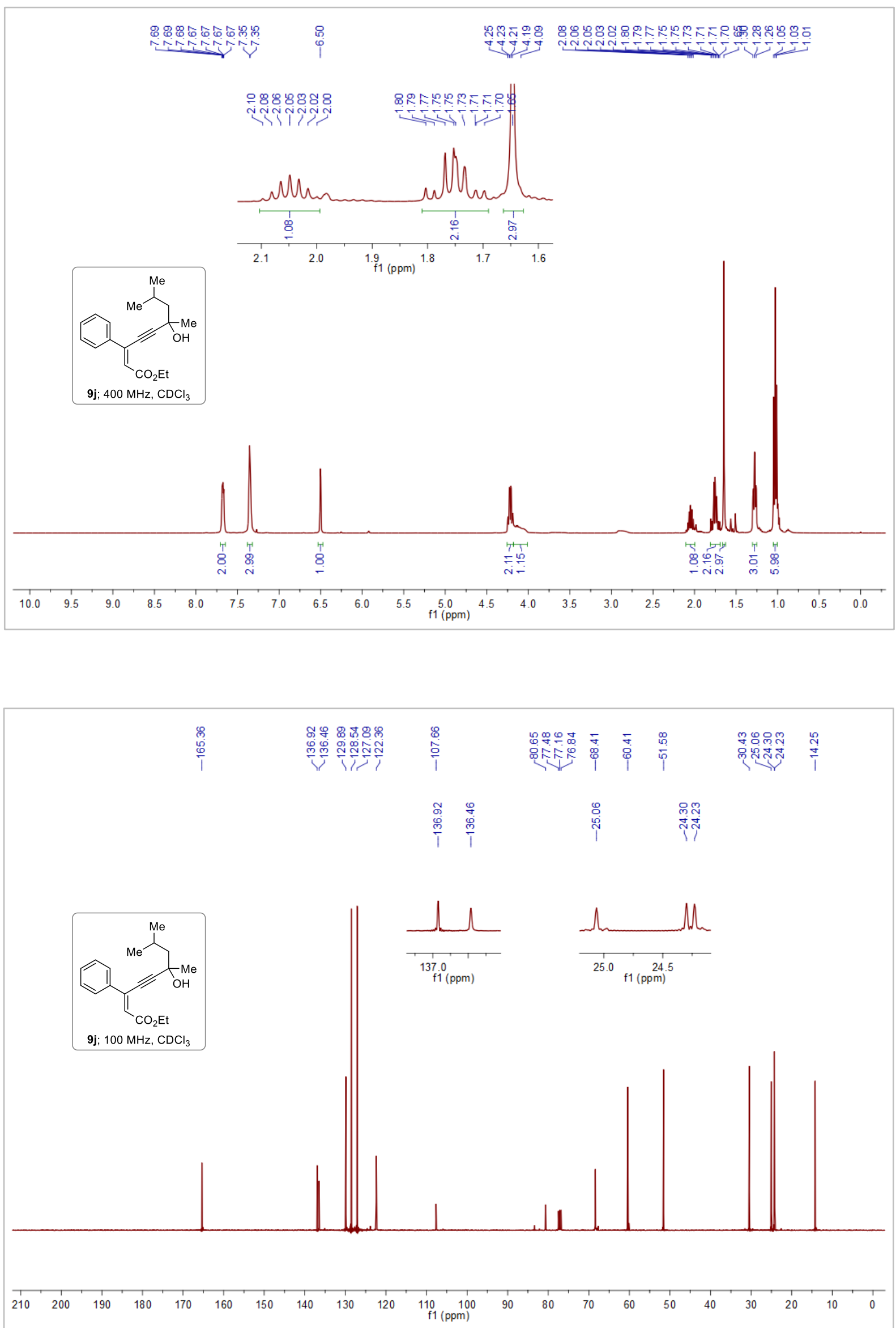
${ }^{1} \mathrm{H}$ NMR $\left(400 \mathrm{MHz}, \mathrm{CDCl}_{3}\right)$ and ${ }^{13} \mathrm{C} \mathrm{NMR}\left(100 \mathrm{MHz}, \mathrm{CDCl}_{3}\right)$ of $9 \mathbf{k}$.
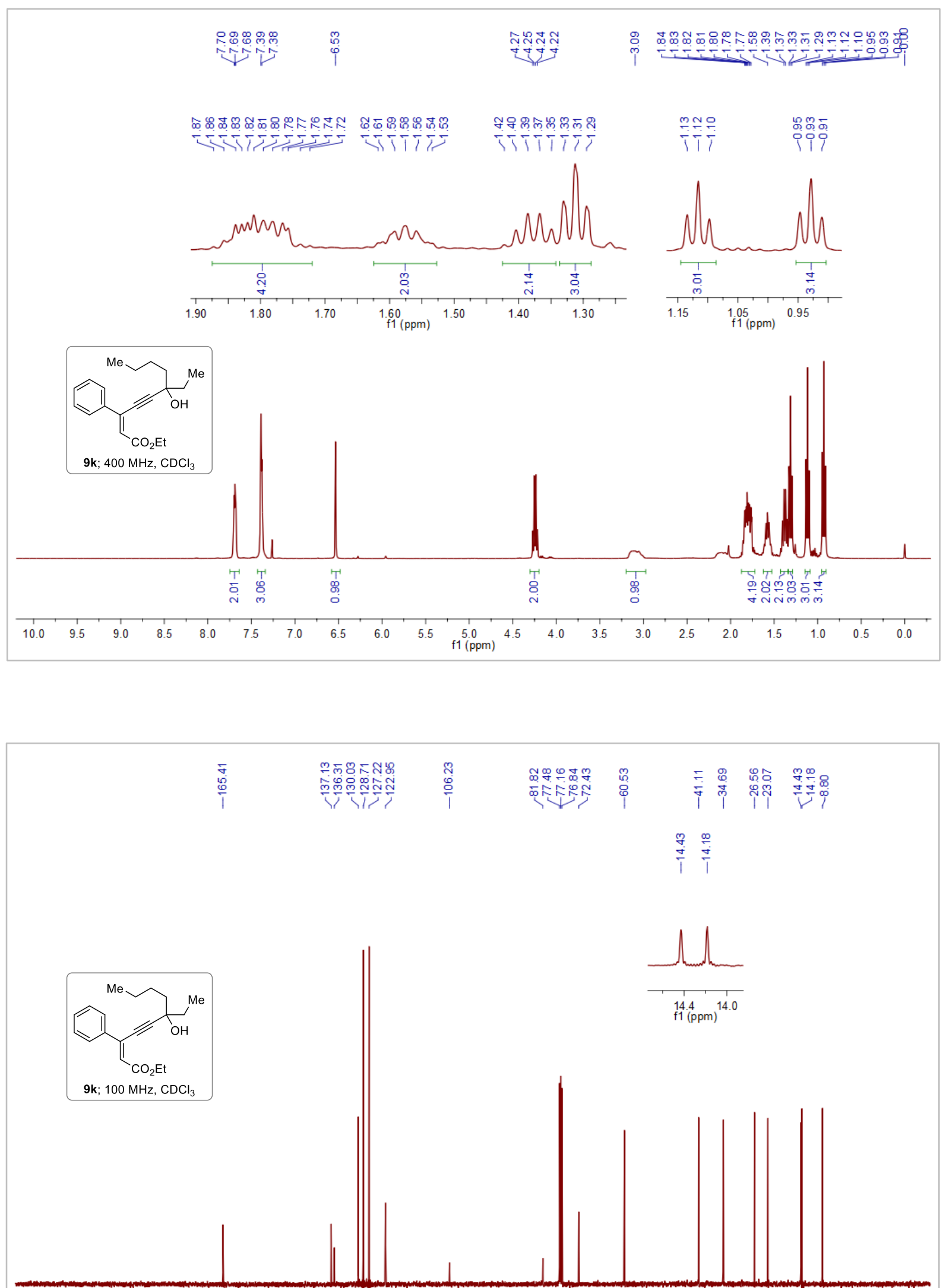

$\begin{array}{llllllllllllllllllllllllllll}210 & 200 & 190 & 180 & 170 & 160 & 150 & 140 & 130 & 120 & 110 & 100 & 90 & 80 & 70 & 60 & 50 & 40 & 30 & 20 & 10 & 0 & -10\end{array}$ 
${ }^{1} \mathrm{H}$ NMR $\left(400 \mathrm{MHz}, \mathrm{CDCl}_{3}\right)$ and ${ }^{13} \mathrm{C} \mathrm{NMR}\left(100 \mathrm{MHz}, \mathrm{CDCl}_{3}\right)$ of 91.
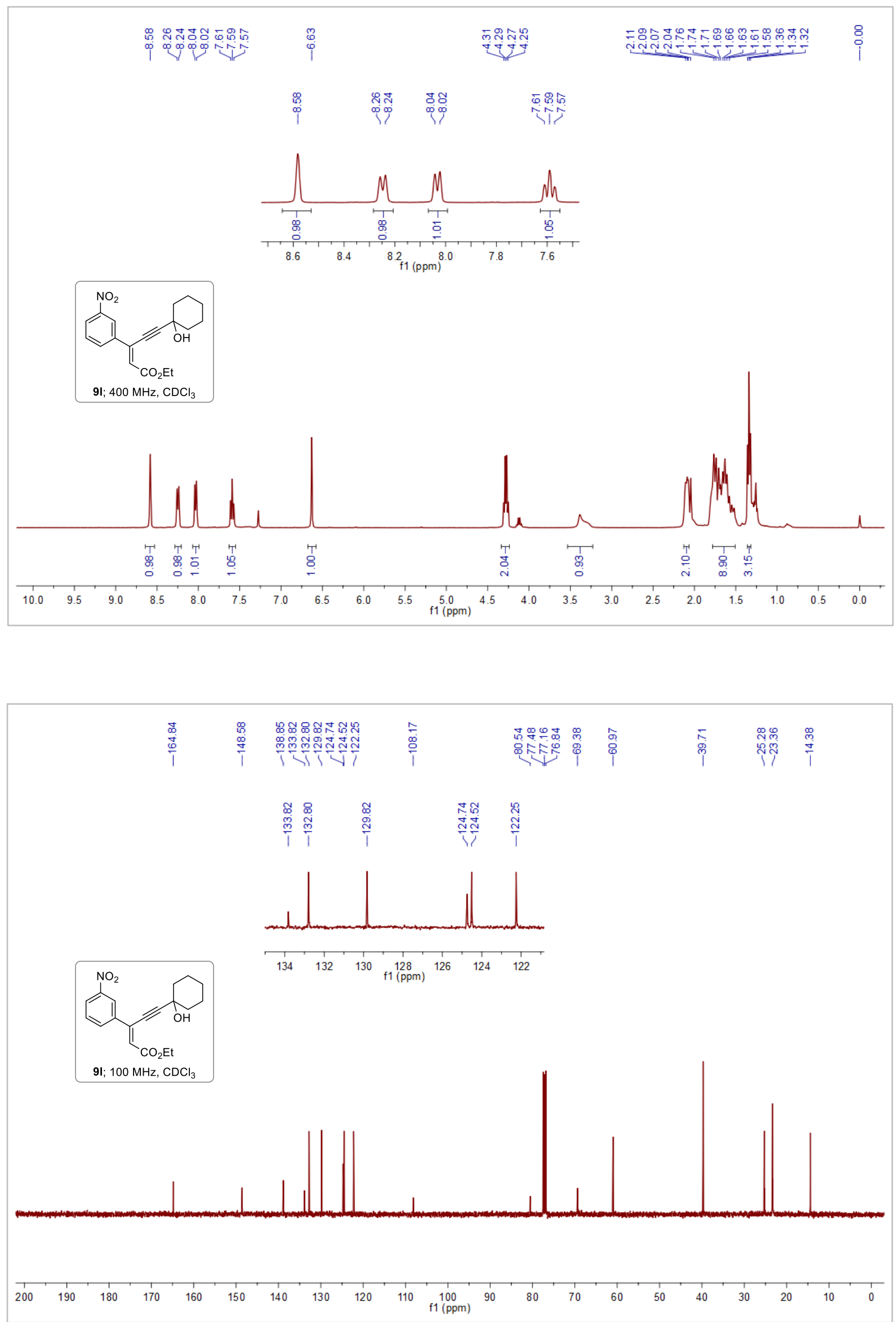
${ }^{1} \mathrm{H}$ NMR (400 MHz, $\left.\mathrm{CDCl}_{3}\right)$ and $\left.{ }^{13} \mathrm{C} \mathrm{NMR} \mathrm{(100} \mathrm{MHz,} \mathrm{CDCl}_{3}\right)$ of 9m.
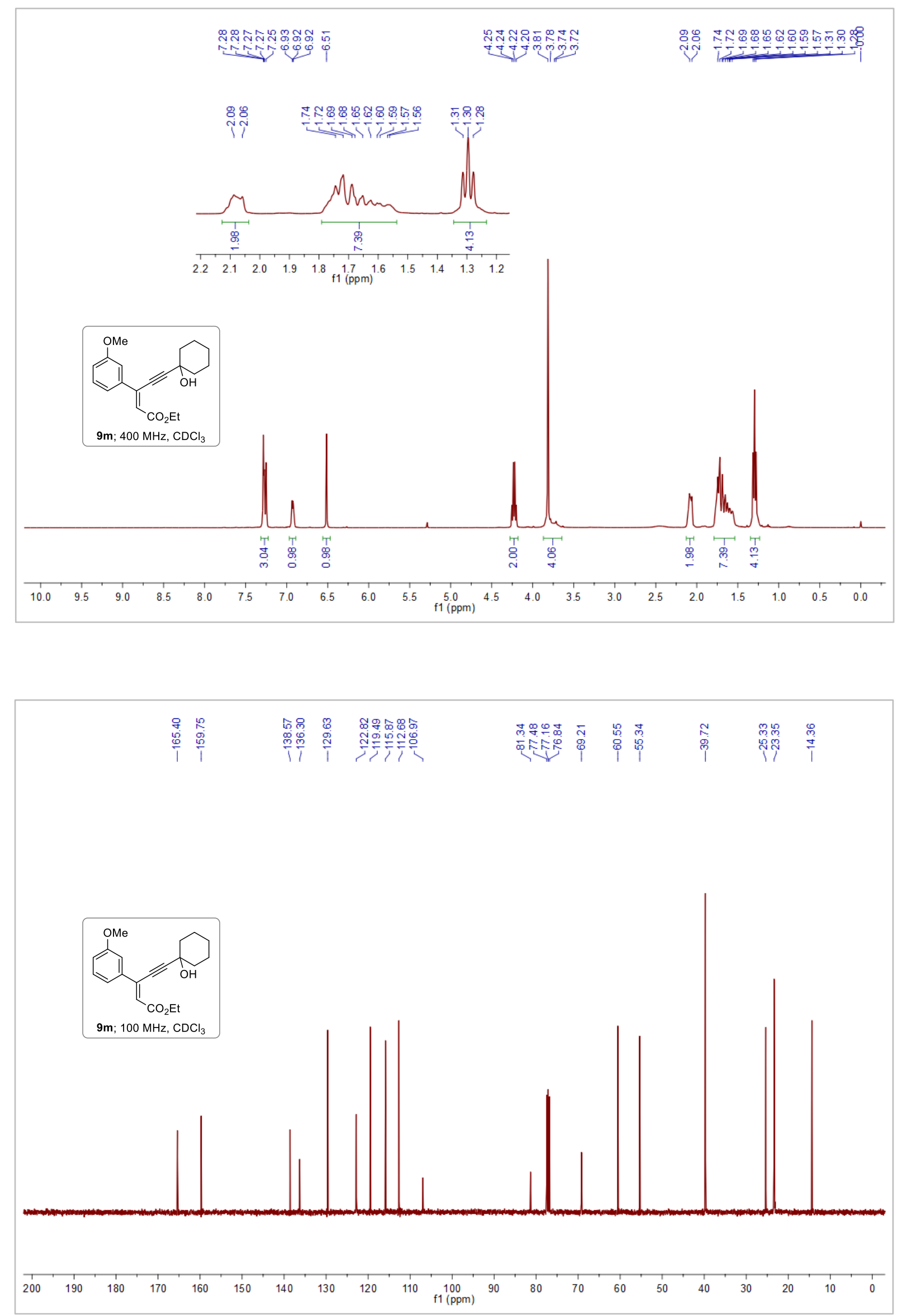
${ }^{1} \mathrm{H} \mathrm{NMR}\left(400 \mathrm{MHz}, \mathrm{CDCl}_{3}\right)$ and ${ }^{13} \mathrm{C} \mathrm{NMR}\left(100 \mathrm{MHz}, \mathrm{CDCl}_{3}\right)$ of 26.
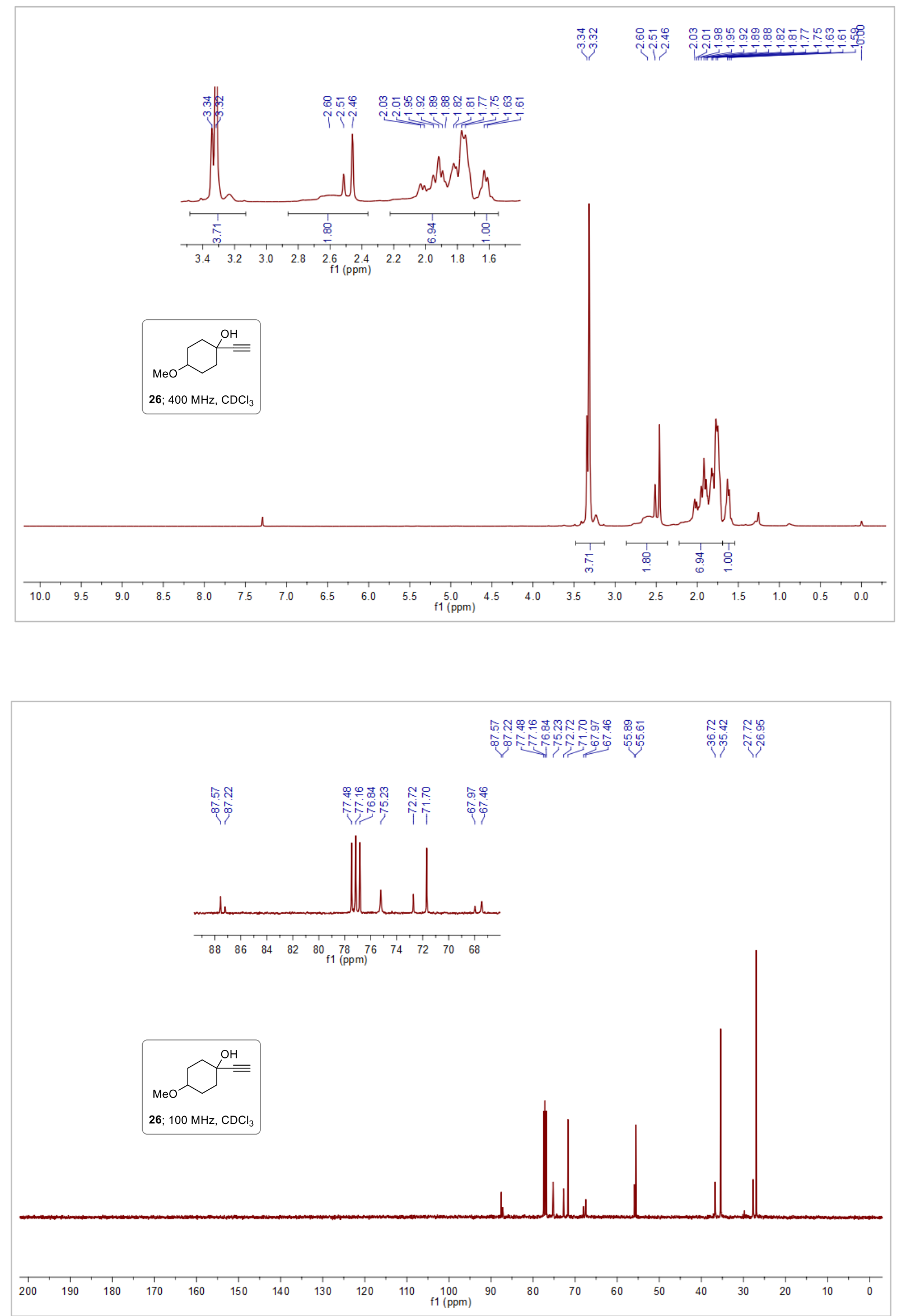
${ }^{1} \mathrm{H}$ NMR $\left(400 \mathrm{MHz}, \mathrm{CDCl}_{3}\right)$ and ${ }^{13} \mathrm{C}$ NMR $\left(100 \mathrm{MHz}, \mathrm{CDCl}_{3}\right)$ of 23.
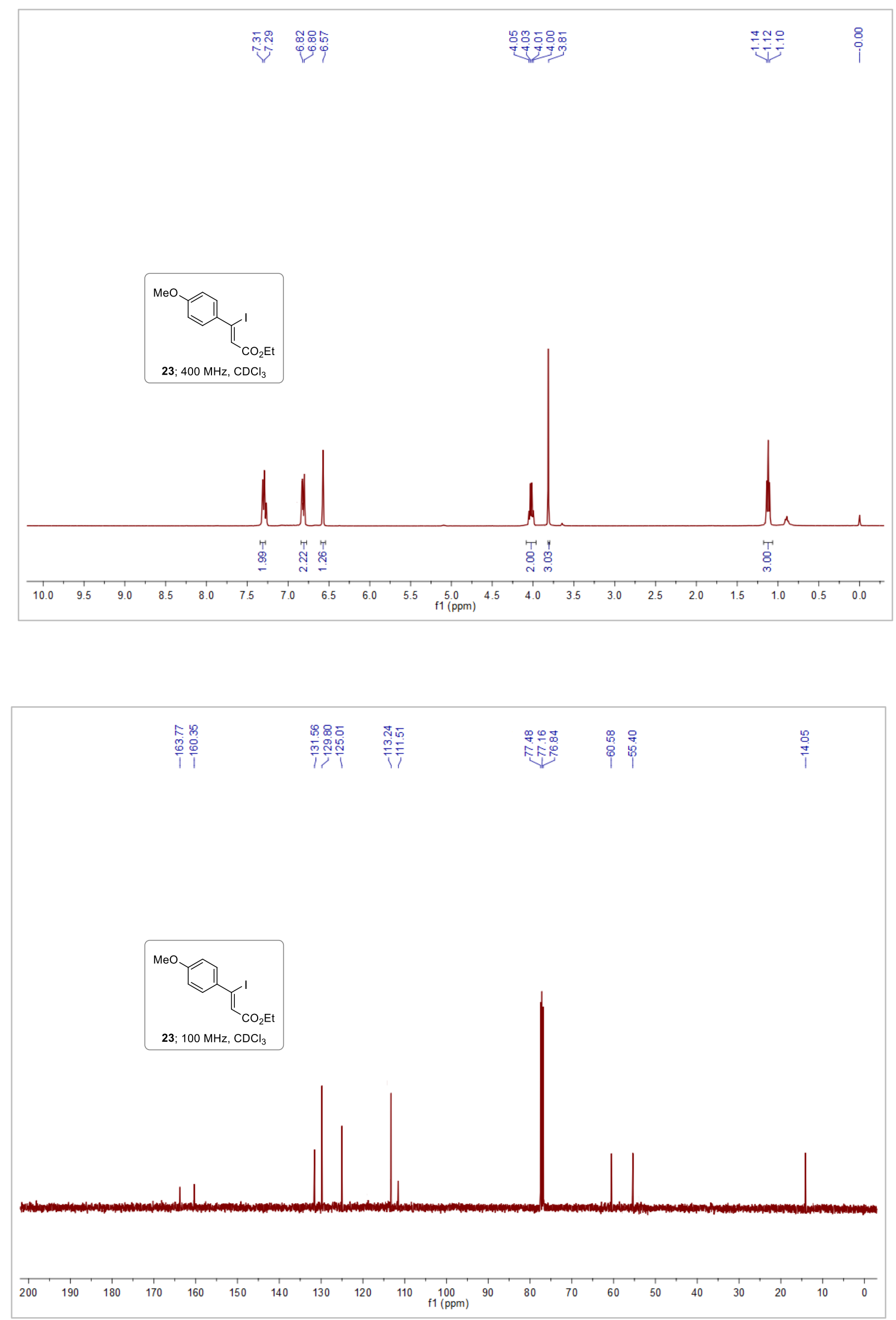

S69 
${ }^{1} \mathrm{H}$ NMR (400 MHz, $\left.\mathrm{CDCl}_{3}\right)$ and ${ }^{13} \mathrm{C} \mathrm{NMR}\left(100 \mathrm{MHz}, \mathrm{CDCl}_{3}\right)$ of 28.
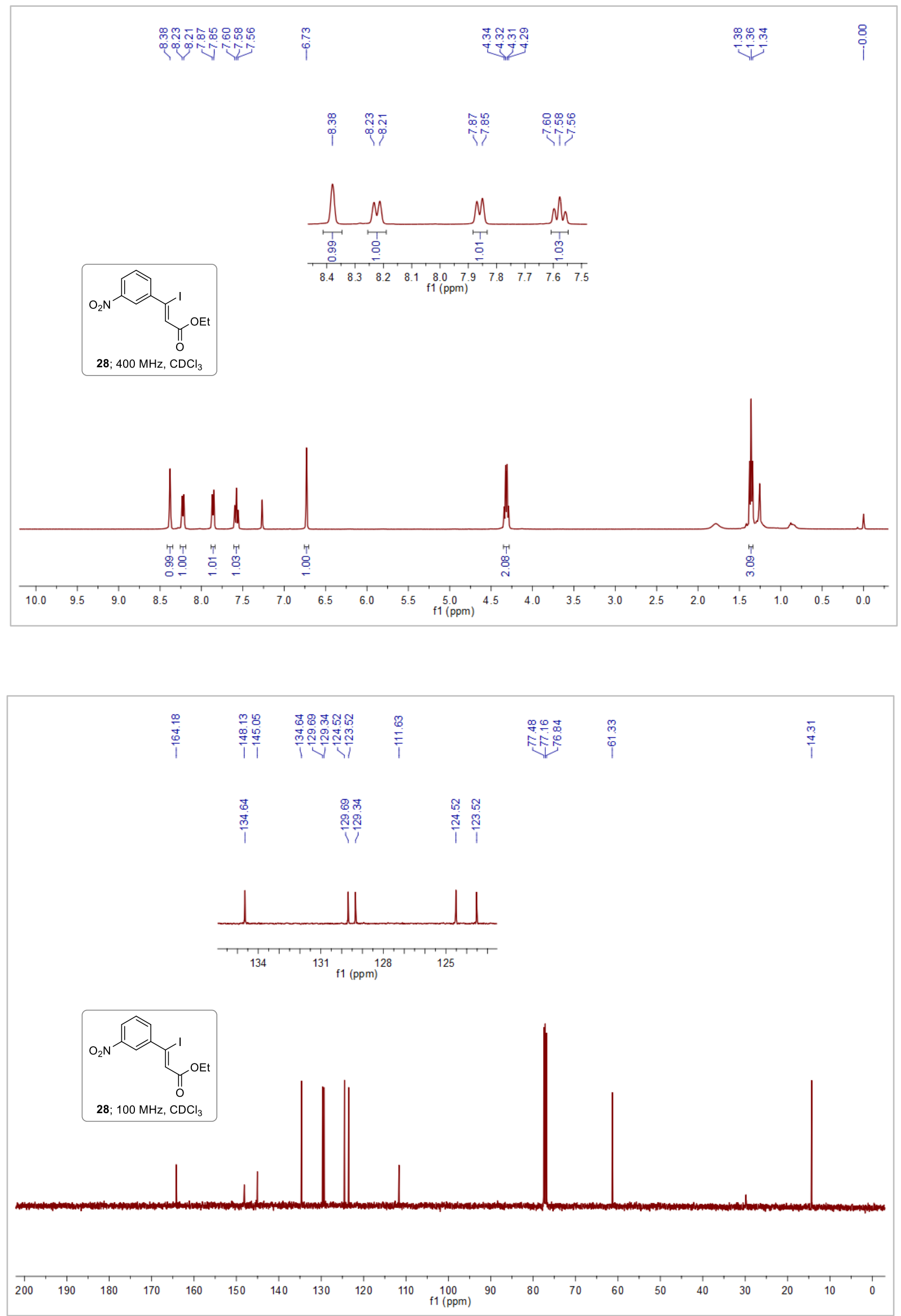